\title{
MULTI-SCALE ASSEMBLY METHODOLOGIES OF POLY(3- HEXYLTHIOPHENE) DERIVATIVE SYSTEMS FOR ENHANCED OPTOELECTRONIC ANISOTROPY
}

\author{
A Thesis \\ presented to \\ the Faculty of California Polytechnic State University, \\ San Luis Obispo \\ In Partial Fulfillment \\ of the Requirements for the Degree \\ Master of Science in Polymers and Coatings Science
}

by

David Bilger

August 2017 
(C) 2017

David Bilger

ALL RIGHTS RESERVED 
TITLE: Multi-Scale Assembly Methodologies of Poly(3-hexylthiophene) Derivative Systems for Enhanced Optoelectronic Anisotropy

AUTHOR: David Bilger

DATE SUBMITTED: $\quad$ August 2017

COMMITTEE CHAIR: Shanju Zhang, Ph.D.

Assistant Professor of Chemistry and Biochemistry

COMMITTEE MEMBER: $\quad$ Chad Immoos, Ph.D.

Professor of Chemistry and Biochemistry

COMMITTEE MEMBER: Erik Sapper, Ph.D.

Assistant Professor of Chemistry and Biochemistry 


\begin{abstract}
Multi-Scale Assembly Methodologies of Poly(3-hexylthiophene) Derivative Systems for Enhanced Optoelectronic Anisotropy

David Bilger
\end{abstract}

Conjugated polymers represent a class of semi-conducting materials with numerous applications in optoelectronic devices, including organic light-emitting diodes, field-effect transistors, and photovoltaics. Because of the numerous advantages of macromolecular systems, including solution processing and mechanical flexibility, conjugated polymers have become a burgeoning field of research with the hopes of producing cost-effective solution-based electronics. Importantly, optoelectronic device performance is heavily influenced by conjugated polymer backbone orientation and overall thin film morphology. As such, the processing conditions of these systems are important to the construction of highperformance optoelectronics. Polythiophenes are model conjugated polymers that have been studied extensively in halogenated organic solvents. However, the selfassembly of these systems from dilute solution to the solid state remains ambiguous for solvents with high dielectric constants.

Here, two derivative compounds of poly(3-hexylthiophene) are correspondingly investigated in high dielectric solvents by way of ultraviolet-visible absorption and fluorescence spectroscopy, Fourier transform infrared spectroscopy, small-angle X-ray scattering, polarized optical microscopy, and fourpoint probe conductivity measurements. In dilute solutions, both systems are found to undergo self-assembly when exposed to various stimuli, including temperature, 
solvent composition, and side-chain characteristics. The kinetics of these transitions are investigated, and a model is put forth to explain contrasting selfassembly mechanisms. At higher concentrations, both systems form lyotropic liquid crystalline phases. Characteristics of the liquid crystalline phases are found to be heavily influenced by dilute solution self-assembly mechanisms and processing. Through the application of a mechanical shear force along still-wet liquid crystal films, alignment of the polythiophene long axis is attained. This morphological characteristic is found to carry over to the solid-state for both systems, and clear optoelectronic anisotropy of the thin films is observed. As such, these methodologies may provide a route to the production of environmentally friendly high-performance optoelectronic devices. 


\section{ACKNOWLEDGMENTS}

Firstly, I would like to acknowledge and thank my advisor, Dr. Shanju Zhang. From the beginning, Dr. Zhang has provided both myself and my peers with every opportunity to be successful in our academic endeavors, whether through research guidance, proposal writing, funding, or simple everyday advice. His determination to see his students succeed is second to none. I am lucky to have worked with him, and I have become a much better individual for it.

Secondly, thank you to Dr.'s Sapper and Immoos for their involvement on my thesis committee, and with my academic career. Throughout my time at Cal Poly, both of these individuals have made themselves available for discussion and advice, and have taken an active interest in my work.

Thirdly, I would like to thank all of those who have worked with me throughout my years at Cal Poly. Specifically, thank you to Amrita Sarkar and Dr. Morgan Stefik at South Carolina University, as well as Dr.'s Manesh Gopinadhan and Chinedum Osuji at Yale University. The collaborative effort between Cal Poly and these institutions gave valuable insight into the chemical systems addressed in this work. Additionally, thank you to my fellow peers, especially Jose Figueroa, Cameron Danesh, Greg Braggin, and Nathan Starkweather for their dedication, hard work, and insight with regards to the presented research.

Finally, thank you to Dr. Fernando and the Polymers and Coatings program, including all the professors associated with it. If not for my involvement with this community, I may never have realized my passion for conducting research, and would likely not have continued furthering my academic career. 


\section{TABLE OF CONTENTS}

Page

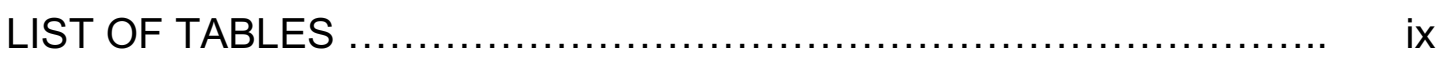

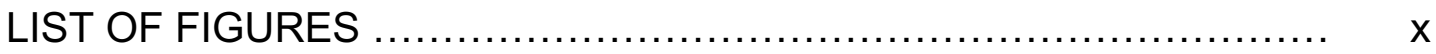

CHAPTER

1. INTRODUCTION ...................................................

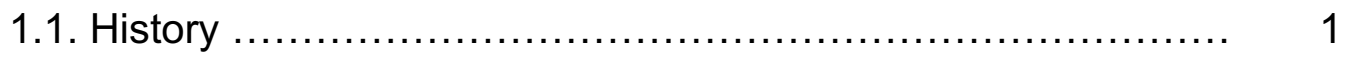

1.2. Conjugated Polymer Applications ............................... 3

1.3. Structure and Conductivity of Conjugated Polymers ............. 8

1.4. Backbone Morphology of Conjugated Polymers ................ 12

1.5. Mechanisms of Polythiophene Self-Assembly ................. 16

1.6. Solution Processing of Conjugated Polymers .................. 18

1.7. Multi-Scale Assembly Methodology of Conjugated Polymers ... 26

2. EXPERIMENTAL METHODS ..................................... 28

2.1. Material Preparation ....................................... 28

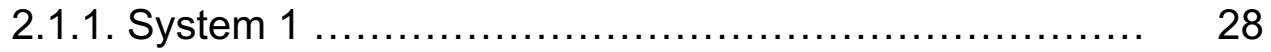

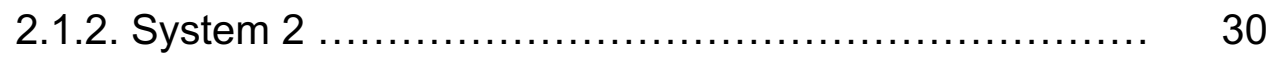

2.2. Instrumental Methods of Characterization ...................... 32

2.2.1. UV-Vis Spectroscopy and Spectrofluorimetry............ 32

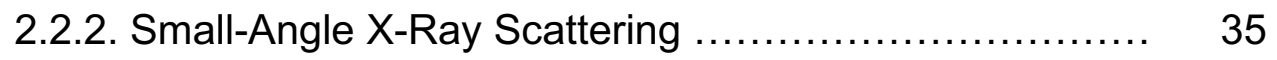

2.2.3. Polarized Optical Microscopy .......................... 38

2.2.4. Fourier-Transform Infrared Spectroscopy ............... 40

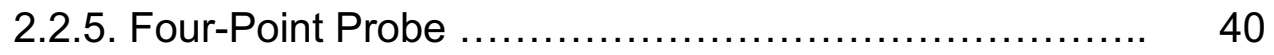


3. RESULTS AND DISCUSSION ..................................... 44

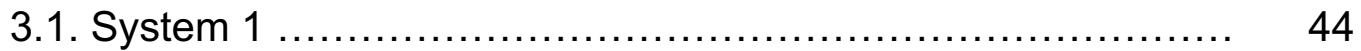

3.1.1. Effect of Side-Chain Architecture and Length ........... 45

3.1.2. Time-Dependent Chromism .......................... 52

3.1.3. Transition Kinetics .................................... 55

3.1.4. Correlation Between ECL and Intrinsic Rigidity ......... 59

3.1.5. Lyotropic Liquid Crystalline Self-Assembly .............. 62

3.1.6. Electrical Properties of P3K6T-Surfactant Thin Films ... 67

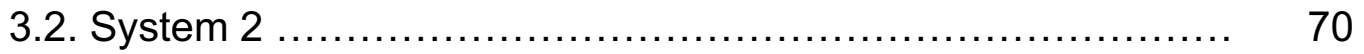

3.2.1. Dilute Solution Properties of P3CnTs ................. 70

3.2.2. Transition Kinetics of P3C5T .......................... 74

3.2.3. Liquid Crystal Gelation of P3C5T ..................... 79

3.2.4. Optoelectronic Anisotropy of P3C5T Thin Films ......... 85

3.3. Research Outlook and Future Work .......................... 88

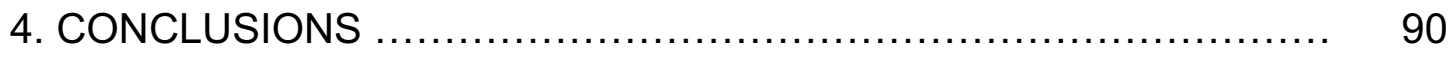

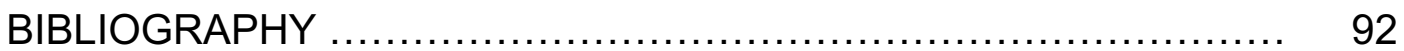




\section{LIST OF TABLES}

Table

Page

1. Calculated Persistence Lengths for P3K6T- STS ${ }_{16}:$ DTS $_{16}$

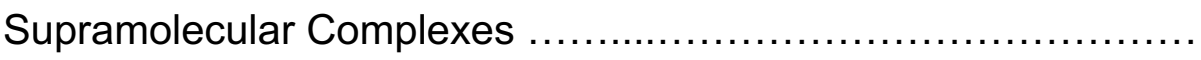

62 


\section{LIST OF FIGURES}

Figure

1. Chemical structures and nomenclature of notable semiconducting polymers ${ }^{7}$

2. Bar chart displaying the conductivity ranges that define insulating, semi-conductive, and conductive materials

3. Diagram depicting the major components of a photovoltaic layer stack. The active layer consists of both a donor and acceptor layer ${ }^{12,13}$

4. Mechanism of charge separation in the active layer. (1) Light absorption leads to exciton formation in the donor material. (2) Excitons diffuse to the donor/acceptor interface. (3) An electron transfers to the acceptor material. (4) An applied electric field separates the polaron pair. (5) The electron and hole are transported to their respective electrodes ${ }^{12}$

5. (a) Ideal heterojunction morphology and (b) bulk heterojunction morphology ${ }^{16}$

6. Illustration of band gap energy levels in insulating, semiconductive, and conductive materials

7. Chemical structure of polythiophene in its oxidatively, reductively, and non-doped states. Oxidative and reductive doping leads to the 
formation of positive and negative quasiparticles, respectively ${ }^{19}$

8. The different directions of 2-D charge transport in organic semiconductors: Intermolecular charge hopping (top) and intramolecular backbone transport (bottom)

9. A polythiophene chain with planarized segments (grey). The total length sum of the grey regions is the effective conjugation length of the chain ${ }^{24}$

10. Two step mechanism of the coil-to-rod transition in poly(3alkylthiophenes) $^{30}$

11. Structural and morphological differences between crystalline

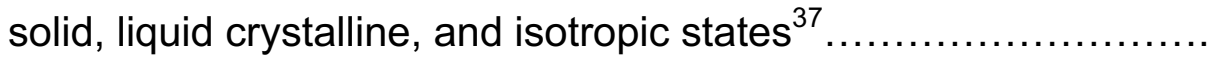

12. Characteristic phase diagrams of aqueous lyotropic surfactant molecules. Below an appropriate temperature solid state crystals begin to form ${ }^{38}$

13. Rod-like particles organized into (a) nematic, (b) smectic $A$, and (c) smectic C LC phases ${ }^{46}$

14. Photos and a schematic illustration of a two-step gelation process

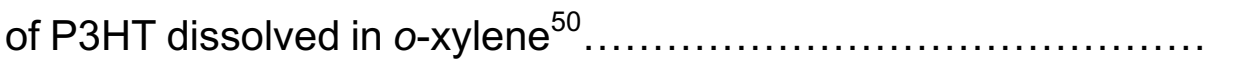

15. Model of CTAB complexed onto the backbone of PTEBS. (b) Chemical structures of the surfactant (CTAB) and the polymer (PTEBS). (c) Depiction of hexagonal packing in the lyotropic LC phase $^{59}$ 
16. (a) Chemical structure of polythiophene P3KnT (system 1) with n $=4,5,6$, or 7 , (b) single-tailed surfactant (STSn) with $n=10,12$, 14 , or 16 , (c) double-tailed surfactant (DTSn) with $n=16$, (d) illustration of the supramolecular complexation of P3KnT with single and double- tailed surfactants, and (e) chemical structure of polythiophene P3CnT (system 2) with $n=3,4,5$, or $6 \ldots \ldots \ldots \ldots . .$.

17. (a) The working mechanism of a UV-vis spectrophotometer. Light passes through an entrance slit and monochromator. Upon leaving the exit slit, monochromatic light passes through the sample and is transmitted to a detector. (b) Working mechanism of a spectrofluorimeter ${ }^{63,64}$.

18. (a) Concentration dependent UV-vis spectra of the 1:1 P3K6T:STS ${ }_{16}$ supramolecular complex at $50^{\circ} \mathrm{C}$. (b) Calibration curve of the 1:1 P3K6T:STS ${ }_{16}$ supramolecular complex

19. Transmission geometry SAXS. An imaginary vector connecting the ends of the collimated and scattered X-ray beams represents the momentum transfer between incident and scattered light ........

20. (a) Optical image displaying a schlerien texture of the nematic LC phase. (b) Working mechanism behind $\mathrm{POM}^{46,66} \ldots \ldots \ldots \ldots \ldots \ldots$

21. (a) Schematic of a four-probe configuration. Current is supplied through the outer probes, and potential is measured by the inner probes. (b) Image of a four-point probe sample stage ${ }^{70}$ 
22. FTIR spectra of pristine P3K6T and 1:1 P3K6T:STS 16 supramolecular complex

23. UV-vis spectra of dilute solution P3KnTs of varying alkyl-chain length ( $\mathrm{n}=4,5,6$, and 7) (a) before and (b) after complexation with STS ${ }_{16}$ over $90 \mathrm{~min}$. The concentration of all solutions is 0.25 $\mathrm{mM}$

24. UV-vis spectra of (a) P3K6T complexed in 1:1 molar ratios with various single tailed surfactants $S T S_{n-1}$ and (b) with various ratios of STS ${ }_{16}:$ DTS $_{16}$. The concentration of all solutions is $0.25 \mathrm{mM}$ and the aging time is $90 \mathrm{~min}$

25. UV-vis spectra of (a) P3K6T complexed in 1:1 molar ratios with (a) various ratios of $\mathrm{STS}_{16}: \mathrm{STS}_{10}$ and (b) various ratios of $\mathrm{STS}_{16}$ : STS $_{14}$. The concentration of all solutions is $0.25 \mathrm{mM}$ and the aging time is $40 \mathrm{~min}$

26. Images of aqueous 1:1 P3KnT:STS ${ }_{16}$ supramolecular complexes at (top) 90 minutes and (bottom) two weeks after dissolution .........

27. Time-dependent chromism of dilute solutions of the supramolecular complexes at $25{ }^{\circ} \mathrm{C}$. (a) UV-vis and (b) fluorescence spectra of 1:1 P3K6T:STS 16 ; (c) UV-vis and (d) fluorescence spectra of P3K6T-9:1 STS $16:$ DTS $_{16}$. The concentration of all solutions is $0.25 \mathrm{mM}$

28. Kinetics of the coil-to-rod transitions of diluted solutions of supramolecular complexes at $25{ }^{\circ} \mathrm{C}$. (a) Time-dependent 
absorbance at $\lambda=590 \mathrm{~nm}$ for 1:1 P3K6T:STS ${ }_{16}$, (b) the dependence of initial rate on polymer concentration for $1: 1$ P3K6T:STS ${ }_{16}$, (c) time-dependent absorbance at $\lambda=590 \mathrm{~nm}$ for P3K6T-9:1 STS $16:$ DTS $_{16}$, and (d) the dependence of initial rate on polymer concentration for P3K6T-9:1 STS 16 :DTS 16

29. Concentrated kinetics of the coil-to-rod transition of supramolecular complexes at $25{ }^{\circ} \mathrm{C}$. (a) Time-dependent absorbance at $\lambda=590 \mathrm{~nm}$ against aging time of $1: 1 \mathrm{P} 3 \mathrm{~K} 6 \mathrm{~T}: \mathrm{STS}_{16}$, (b) the dependence of initial rate on polymer concentration for 1:1 P3K6T:STS

30. Room temperature SAXS profiles of aqueous solutions of P3K6T supramolecular complexes $(\sim 0.60$ wt $\%)$ with different

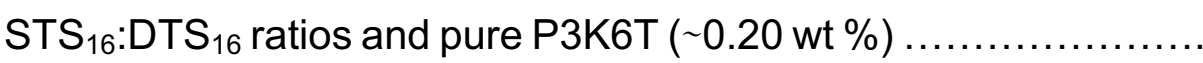
60

31. Optical images of $51 \mathrm{wt} \%$ aqueous solutions of the 1:1 P3K6TSTS $_{16}$ supramolecular complex under crossed polarizers. (a) Without mechanical shearing and (b) with shearing. The arrow in part $b$ indicates the shear direction .................................

32. Room-temperature liquid crystalline phase diagrams of P3K6T supramolecular complexes with (a) varying chain lengths of single tailed surfactants $\left(S T S_{n-1}\right)$ and (b) varying double tailed surfactant

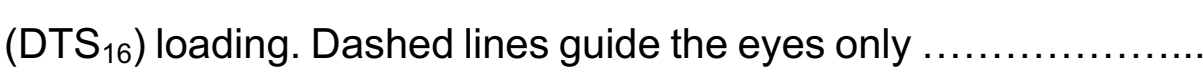

33.(a) Room temperature SAXS pattern and (b) temperature dependent in situ SAXS profile of the lyotropic solution of the 1:1 
P3K6T-STS ${ }_{16}$ supramolecular complex. The concentration of the solution is $\sim 51 \mathrm{wt} \%$

34. Optical images of a sheared LC film of P3K6T-STS16 complex under crossed polarizers after drying. (a) $0^{\circ}$ rotation and (b) $45^{\circ}$ rotation. The arrows represent the shear direction

35. Room-temperature measurements of anisotropic conductivity. (a) Illustration of electrical conductivity measurements and (b) current vs voltage curves of sheared P3K6T-STS ${ }_{16}$ LC films after complete drying parallel and perpendicular to the shear direction.

36. UV-vis spectra of dilute P3CnTs in DMSO with varied alkyl-chain lengths ( $n=3,4,5$, and 6$)$ after 80 -minute equilibration. The concentration of solutions is $25 \mu \mathrm{g} / \mathrm{mL}$

37. UV-vis spectra (a) upon and (b) 24 hours after initial dissolution of P3C5T in varying ratios of DMSO:EtOH. The concentration of all solutions is approximately $25 \mu \mathrm{g} / \mathrm{mL}$

38. UV-vis spectra of P3C5T thermochromism in (a) DMSO and (b) 8:2 DMSO:EtOH. The concentration of all solutions is approximately $25 \mu \mathrm{g} / \mathrm{mL}$

39. Time-dependent chromism of dilute solutions of P3C5T in (a) DMSO and (b) 8:2 DMSO:EtOH. The concentration of all solutions is approximately $25 \mu \mathrm{g} / \mathrm{mL}$ 
40. Assembly kinetics of diluted solutions of $\mathrm{P} 3 \mathrm{C} 5 \mathrm{~T}$ at $25^{\circ} \mathrm{C}$. (a) Timedependent absorbance against aging time of P3C5T in DMSO, (b) the initial rate versus polymer concentration of P3C5T in DMSO, (c) time-dependent absorbance against aging time of P3C5T in 8:2 DMSO:EtOH, and (d) the initial rate versus polymer concentration of P3C5T in 8:2 DMSO:EtOH

41. FT-IR spectra showing the carbonyl shifting between P3C5T gels and solid films. The concentration of the gel is $30 \mathrm{wt} \%$..............

42.(a) Bulk and (b) thin film characteristics of concentrated P3C5T/DMSO thermoreversible gels. The concentration of all thin films is $23 w t \%$

43. Phase diagram of P3C5T in DMSO LC formation as a function of concentration. Illustrative optical images of isotropic and liquid crystalline phases are displayed above the phase boundary ........

44. Optical images of periodic stripe textures of P3C5T in DMSO at (a) 5x and (b) 10x magnification showing the periodicity of P3C5T banded textures

45. Optical images of sheared films of gelled P3C5T in DMSO under crossed polarizers. (a) $0^{\circ}$ rotation and (b) $45^{\circ}$ rotation of crossed polarizers. The solution concentration is $23 \mathrm{wt} \%$ and the shear direction is parallel to the single arrows

46. (a) Optical images showing alterations of transmission intensity of sheared P3C5T films as a function of sample rotation. Plots of 
Transmission intensity as a function of sample rotation in (b) Cartesian and (c) polar coordinates illustrating optical anisotropy of sheared P3C5T LC gel films. For both plots, normalization was done over the entire range of data shown

47. Room-temperature measurement of anisotropic conductivity. (a) Current versus voltage curves of sheared P3C5T LC gel films after complete drying with probes parallel and perpendicular to the shear direction. (b) Bar graph comparing the anisotropic conductivity of P3C5T LC gel films in parallel and perpendicular orientations relative to the direction of shear ........................ 


\section{Introduction}

\subsection{History}

Polymeric materials have been employed extensively by human beings over the course of our existence. In pre-historic times, these materials were exclusively bio-derived, mostly in the form of plant fibers, wood, bone, or skin. Currently, polymers are not only derived from nature, but also by synthetic means. Despite our rich history with polymeric materials and their applications in everyday life, polymer science, from a molecular standpoint, is an advance with origins in the twentieth century. The concept of macromolecules was first developed by Hermann Staudinger in the 1920's'. Eventually, scientific inquires provided the data necessary to prove Staudinger's theories, and he was subsequently awarded the Chemistry Nobel Prize in 1953 "for his discoveries in the field of macromolecular chemistry" ${ }^{\prime 1}$. Eighteen years' prior, Wallance Corothers invented Nylon while undertaking research for DuPont ${ }^{2}$. The findings of Corothers demonstrated the significant potential of synthetic polymers on an industrial scale, and not long after this did Karl Ziegler and Giulio Natta's discoveries of polymerization catalysts shape the current polymers industry. In 1963, Ziegler and Natta jointly shared the Chemistry Nobel Prize "for their discoveries in the field of the chemistry and technology of high polymers" ${ }^{3}$. Finally, Paul Flory provided the necessary theoretical explanations of macromolecular phenomena through his experimental studies outlined in his 1953 publication, Principles of Polymer Chemistry. Flory was awarded the Chemistry Nobel Prize in 1974 "for his 
fundamental achievements, both theoretical and experimental, in the physical chemistry of macromolecules"4.

While the works of Staudinger, Flory, Ziegler, and Natta provided numerous advances to the field of polymer science, the specific materials investigated by these men consisted of saturated $\mathrm{sp}^{3}$ hybridized carbon backbones, resulting in non-conductive properties. In 1977, Alan MacDiarmid, Hidekii Shirakawa, and Alan Heeger experimented with non-saturated $\pi$-conjugated macromolecules, and were successful in doping these materials over an extensive range of conductivities, from insulators to metals ${ }^{5}$. Prior to this, conjugated polymers were of little interest due to their poor properties, including insolubility and minimal conductivity. As such, MacDiarmid, Shirakawa, and Heeger were jointly awarded the Chemistry Nobel Prize in 2000, "for the discovery and development of electrically conductive polymers ${ }^{6}$." This paved the way for new and exciting research at the interface of materials chemistry and condensed matter physics.

Shortly after the advent of conductive conjugated polymers, the study of such systems began expanding into many diverse fields. Notably, the inherent connection between the charge transport and chemical structure of conjugated polymers began attracting the attention of synthetic chemists ${ }^{6}$. In the 1980 's, large quantities of various $\pi$-conjugated macromolecules began being synthesized, and resulted in alterations of conductivity, mechanical properties, and solution processing $^{6}$. Therefore, conjugated polymers not only exhibit the optical and charge carrier properties of metals, but simultaneously maintain the attractive 
processing capabilities of polymers. Accordingly, extensive efforts have been paid to characterizing these materials for use in numerous applications.

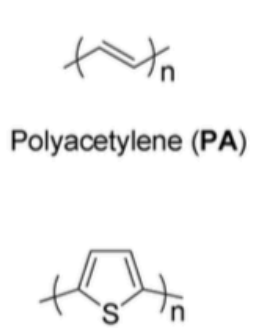

Polythiophene (PT)

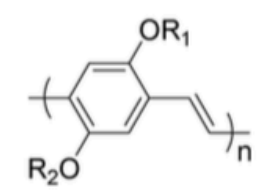

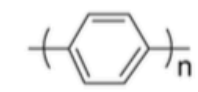

Polyparaphenylene (PPP)

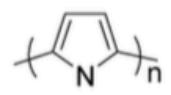

Polypyrrole (PPy)

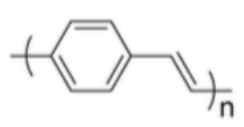

Polyparaphenylene vinylene (PPV)

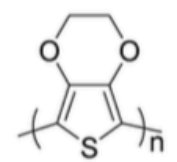

Polyethylene dioxythiophene (PEDOT)

Poly(2,5 dialkoxy) paraphenylene vinylene (e.g.MEH-PPV) Poly(3-alkyl) thiophene (P3AT) (R-methyl, butyl, etc.)

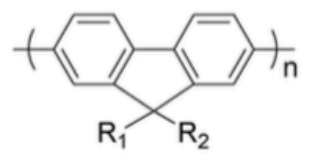

Polyfluorene (PFO)

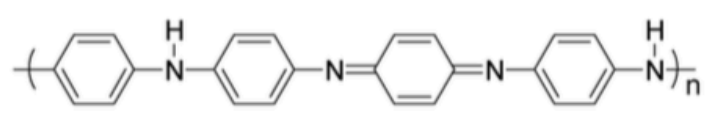

Polyaniline (PANI)

Figure 1. Chemical structures and nomenclature of notable semi-conducting polymers ${ }^{7}$.

\subsection{Conjugated Polymer Applications}

Though various types of electronic devices exist in which conjugated polymers are applicable, including light-emitting diodes and field-effect transistors, photovoltaic cells have become an exceedingly interdisciplinary and burgeoning field of research due to their numerous complexities. Currently, inorganic materials represent the industry standards with regards to photovoltaic devices ${ }^{8}$. This is due to these materials possessing crystalline structures with long-range periodicity, which allows for greater electronic device efficiency. Electrically, two disparities 
exist between organic and inorganic conductors. First, charge carriers can move freely throughout the conjugated backbone of organic materials, but intermolecular transport through charge hopping is hindered ${ }^{8}$. This limits the charge transport capabilities of organic materials compared to that of inorganic counterparts. Fortunately, organic materials possess large absorption coefficients, allowing for the efficient capture of incoming photons ${ }^{9}$. Secondly, Frenkel excitons (electronhole pairs) have been observed as the main charge carriers in organic materials, which makes electron extraction difficult in photovoltaic devices due to the strong interactions between electrons and their respective holes ${ }^{8}$. In contrast, inorganic materials contain excitons of the Wannier-type, which allow for efficient separation of electrons and holes due to binding energies that are on the same order as room temperature $^{10}$. Despite these comparisons, inorganic electronics still retain numerous pit-falls with regards to processing, including expensive vapor deposition techniques. Fortunately, where common inorganic materials have failed, conjugated polymers have the potential to succeed.

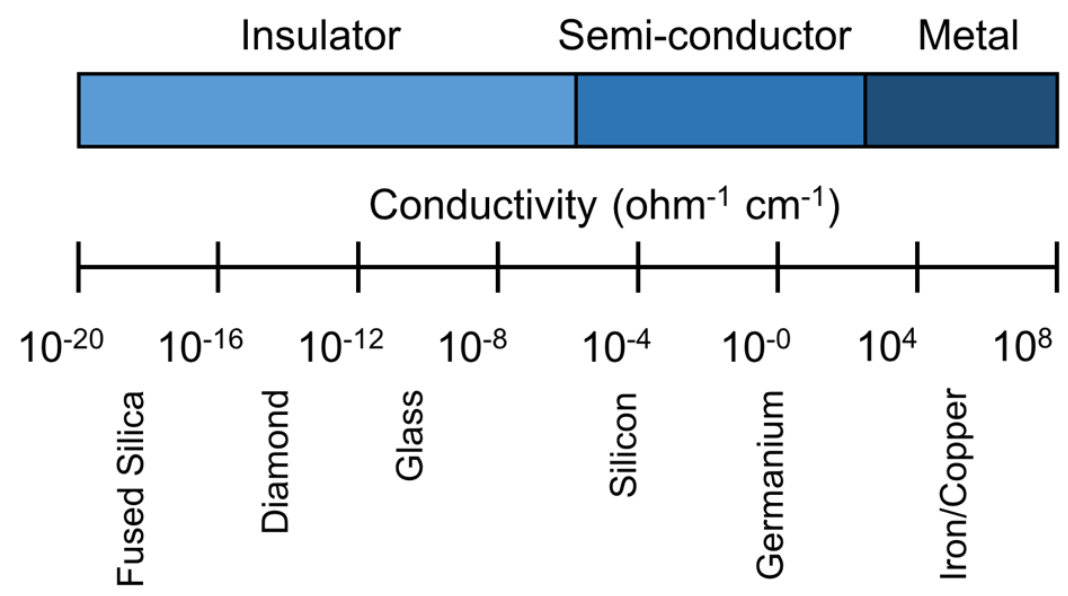

Figure 2. Bar chart displaying the conductivity ranges that define insulating, semiconductive, and conductive materials. 
Because the electron transfer interactions between neighboring chains of conductive polymers are relatively strong compared to the intermolecular attractions of non-conjugated systems, conductive polymers tend to be insoluble. Luckily, several chemical structures of conjugated systems have been synthesized with the capacity to mitigate solubility issues ${ }^{7}$. An example of this may be noted in the structure of poly(3-alkylthiophenes) (P3AT), in which the incorporation of reasonably long side chains onto the thiophene monomer units allows for solubility in numerous organic solvents. As such, the capacity to produce electronic devices of conducting polymers via solvent-based coatings processes, such as roll-to-roll printing, has become a distinct reality. Currently, two of the most high-throughput methods for processing conductive polymers are flexographic printing and rotary screen printing ${ }^{11}$. While solution-based processing has been successful in producing electronic devices from conductive polymers, the average device efficiencies are considerably low. As such, current research on conductive polymers seeks to increase electronic device efficiency while still maintaining costeffective solution-based processing.

The actual building of a polymer solar cell requires the construction of a layer stack, such as the one presented in Figure 3. After a conjugated polymer is dissolved in an appropriate solvent, it is then transferred to a substrate through one of the aforementioned coatings processes. The necessary materials are layered on top of each other to construct the solar cell stack. Such a layer stack consists of five components: an active layer, a hole transport layer, an electron transport layer, a positive electrode, and a negative electrode ${ }^{12}$. Importantly, the 
active layer consists of two sections, a donor and acceptor material, that act to effectively separate tightly bound Frenkel excitons ${ }^{8,12}$.

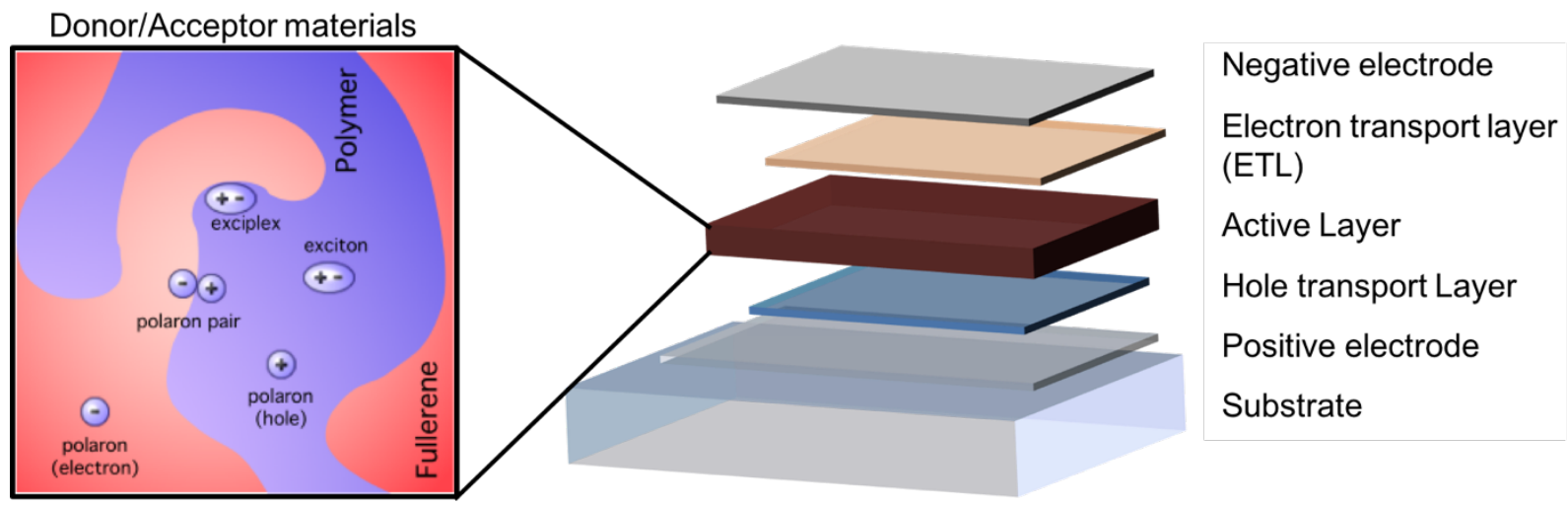

Figure 3. Diagram depicting the major components of a photovoltaic layer stack.

The active layer consists of both a donor and acceptor layer ${ }^{12,13}$.

The donor material (conjugated polymer) absorbs light and produces the bound electron-hole pair, or exciton. Eventually, the exciton reaches the donor/acceptor material interface, and the electron is then extracted by the acceptor material. After extraction, the electron and hole each diffuse to their respective electrodes. If the donor/acceptor interface is not reached within a few tens of nanometers, then the exciton will recombine and the solar cell efficiency will be suppressed ${ }^{14}$. An illustrative description of this process may be seen in Figure 4. Therefore, the composition and arrangement of the layer stack, including the morphology of the active layer, can all have an effect on the overall photovoltaic device efficiency ${ }^{14}$. As such, numerous techniques have been employed to improve device performance, and have ultimately led to device efficiencies of up to $10 \%{ }^{15}$. 

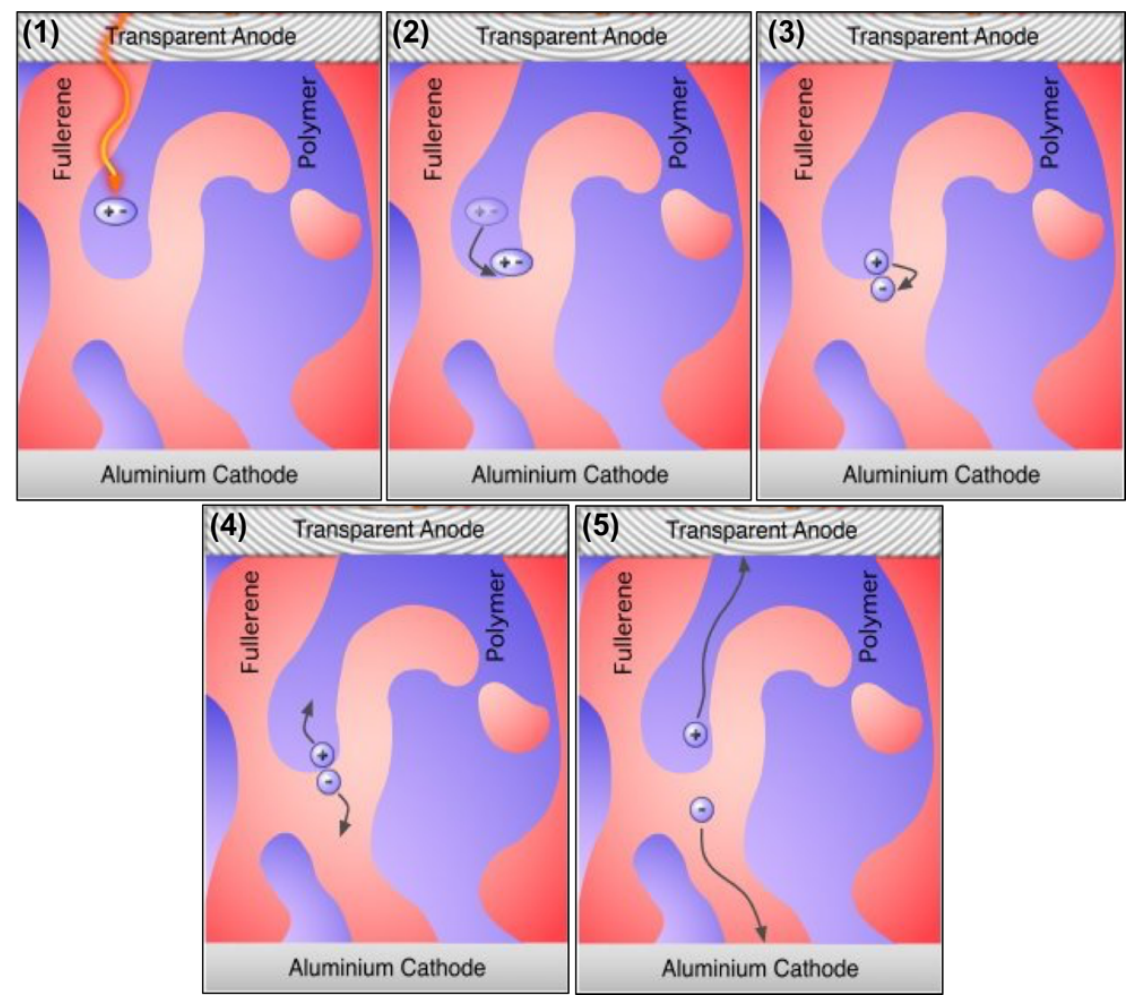

Figure 4. Mechanism of charge separation in the active layer. (1) Light absorption leads to exciton formation in the donor material. (2) Excitons diffuse to the donor/acceptor interface. (3) An electron transfers to the acceptor material. (4) An applied electric field separates the polaron pair. (5) The electron and hole are transported to their respective electrodes ${ }^{12}$.

In organic photovoltaics, the preferred active layer morphology is referred to as a bulk heterojunction, which is formed through the mixing of donor and acceptor materials. The ideal blended morphology entails a bi-continuous interpenetration network, which is displayed in Figure 5a. In other words, domains of both donor and acceptor materials have sizes of twice the exciton diffusion length $(\sim 20 \mathrm{~nm})$ in order to promote exciton separation by encouraging quick translation to the donor/acceptor interface ${ }^{8,14}$. This ideal blended morphology of 
the bulk heterojunction is commonly referred to as a comb-like structure. Aside from controlling morphologies of the active layer, significant interest has been applied to tailoring morphologies of the individual donor and acceptor materials ${ }^{14,16}$. This is particularly true of donor materials such as poly(3hexylthiophene), in which more ordered crystalline arrangements have yielded improved device efficiencies ${ }^{17}$. Notably, such an effect stems from the dependence of electronic character on the conjugated backbone arrangements of organic materials.

(a)

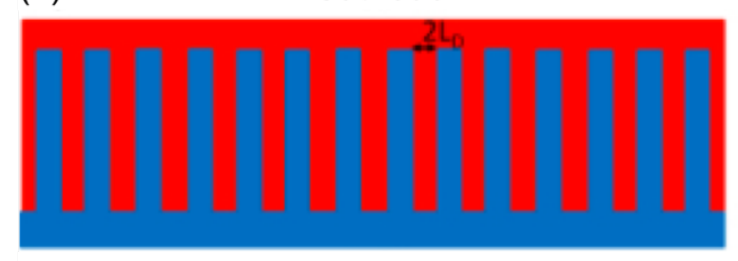

Anode

Cathode

Donor (b)

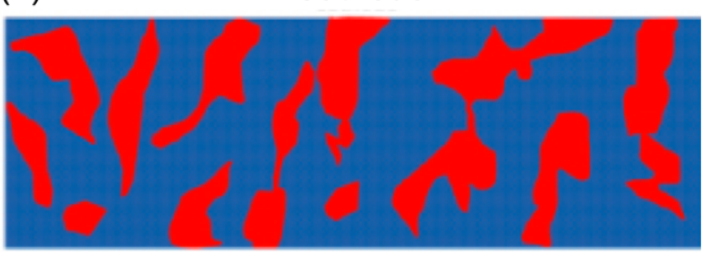

Anode

Cathode

\section{Acceptor}

Figure 5. (a) Ideal heterojunction morphology and (b) bulk heterojunction morphology ${ }^{18}$.

\subsection{Structure and Conductivity of Conjugated Polymers}

The mechanism of charge transport in organic macromolecules originates from the existence of conjugated $\pi$ bonds along the polymer backbone. This alteration of adjacent double and single bonds produces polymers with continuous $\mathrm{sp}^{2}$ hybridized carbon centers. As such, one valence electron at each center resides in a $p_{z}$ orbital that is directionally perpendicular to the three remaining sigma bonds. Therefore, the combination of the remaining $p_{z}$ orbitals allows for 
electrons to delocalize throughout the conjugated polymer main chain. However, in organic materials, the high binding energy of electrons makes them difficult to separate from their respective orbitals. The energy required to overcome the potential barrier mentioned above is referred to as the band gap, and allows electrons to be excited from a valence band to a conduction band ${ }^{7}$. In organic materials, the valence and conduction bands are referred to as the highest occupied molecular orbital (HOMO) and lowest unoccupied molecular orbital (LUMO), respectively ${ }^{7}$. The alteration of the band gap energy is what distinguishes insulating, conducting, and semi-conducting materials, as shown in Figure 6. In inorganic conductors, the band gap is minimal to non-existent, and therefore electrons may move freely in three dimensions due to the even spacing of molecular orbitals in all directions. In contrast, the continuity of conjugated polymers is variable, and the charge transport may be considered one dimensional.

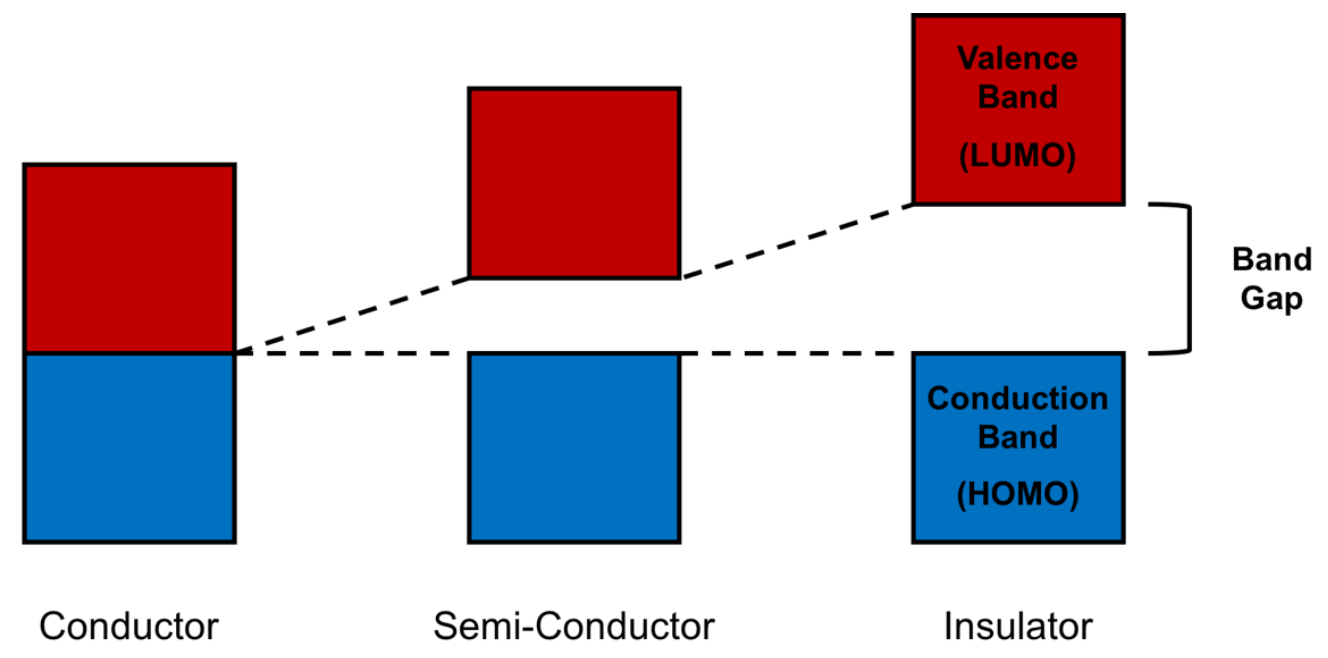

Figure 6. Illustration of band gap energy levels in insulating, semi-conductive, and conductive materials. 
Due to the variable continuity and large binding energies of conjugated polymers, the materials exhibit inherent semi-conductive properties with increased band gaps compared to their inorganic counterparts. In order to mitigate these properties, chemical doping of the conjugated polymer backbone is commonly employed to produce a net charge ${ }^{19}$. The charge may be either positive or negative for oxidative or reductive doping, respectively. Halogens or alkali metals are commonly used to dope conjugated polymers; as is the case with poly(3dodecylthiophene), which sustains conductivities that approach $1000 \mathrm{~S} / \mathrm{cm}$ when doped with iodine ${ }^{20}$. While this value is still relatively small compared to a metal such as copper $\left(5 \times 10^{5} \mathrm{~S} / \mathrm{cm}\right)$, few applications of conjugated polymers require conductivities greater than $1000 \mathrm{~S} / \mathrm{cm}$. The mechanism of how doping increases charge mobility results from interactions between opposing charges, which may be seen in Figure 7. For example, when a conjugated material is doped via oxidation, an electron is removed from the backbone, producing a positive polaron. The positive polaron has strong interactions with the couter-ion of the corresponding dopant. As such, localization and mobility of the polaron is strongly coupled to that of the dopant counterion. Therefore, increasing dopant concentration simultaneously increases polaron mobility and macroscopic conductivity of the polymer. However, macroscopic charge-transport of conjugated polymers is dependent on multiple factors besides the dopant and dopant concentration. 


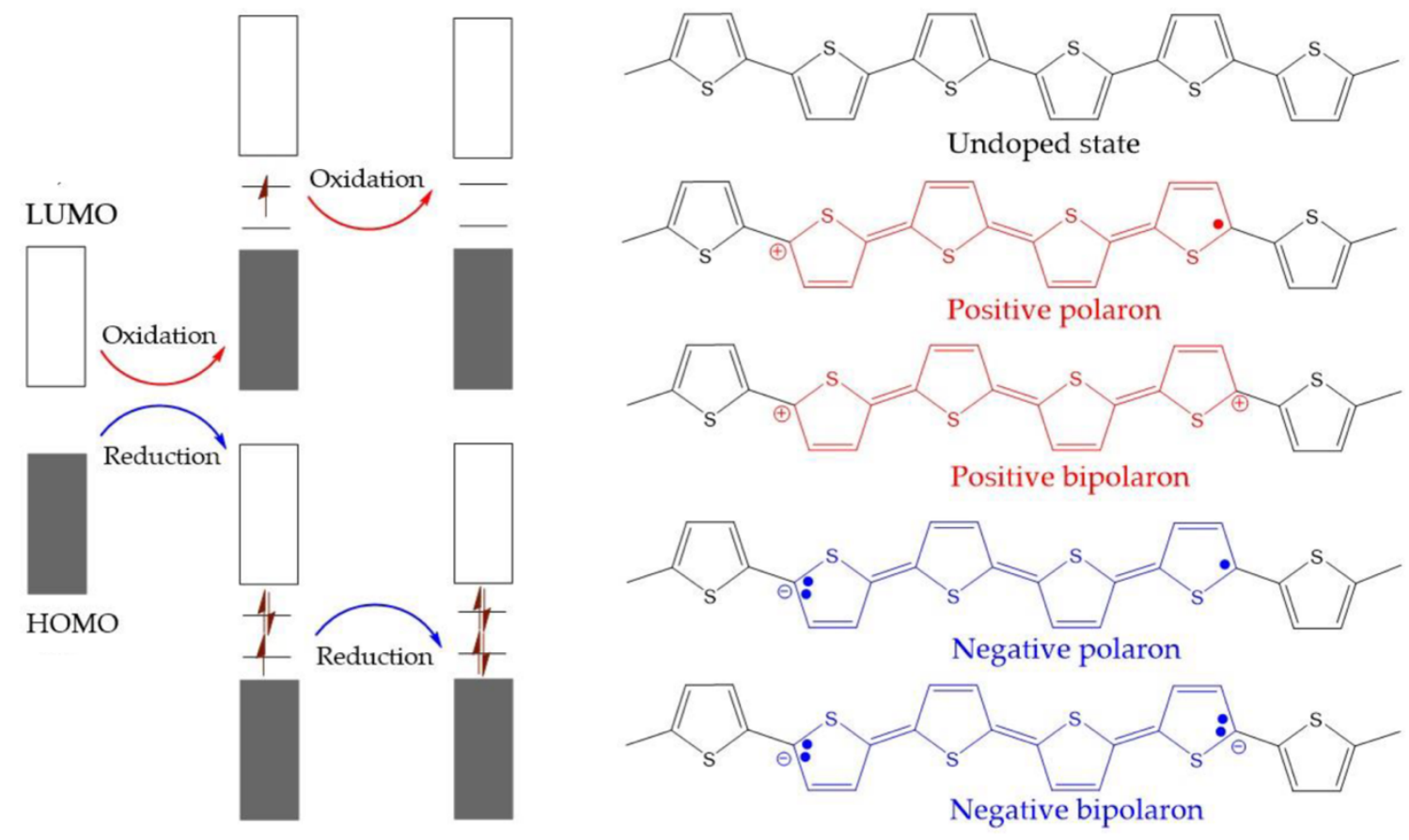

Figure 7. Chemical structure of polythiophene in its oxidatively, reductively, and non-doped states. Oxidative and reductive doping leads to the formation of positive and negative quasiparticles, respectively ${ }^{19}$.

From a quantum mechanical perspective, the band gap energy of conjugated polymers may be modeled as a particle that can move freely on an infinitely thin wire. This model is appropriate as intra-chain charge transport is a one dimensional phenomenon. In such a model, the ends of the wire are obstructed by infinitely large potential wells, and the potential energy of the freemoving particle along the wire is zero. For this system, solutions to the timeindependent Schrodinger equation (after the application of boundary conditions) indicate the particles wavefunction yields discrete energy levels. The energy eigenvalues of such a system may be calculated using equation 1 


$$
E_{n}=\frac{n^{2} \hbar^{2}}{8 m_{e} L^{2}}, \text { where } n=1,2,3 \ldots
$$

where $\mathrm{n}$ is the energy level, $\hbar$ is Plank's constant divided by $2 \pi, \mathrm{m}_{\mathrm{e}}$ is the mass of an electron, and $L$ is the length of the infinitely thin wire. In principle, the energies of the HOMO and LUMO energy levels may be calculated using equation 1, and the absolute difference between them will yield a theoretical value of the band gap. Intriguingly, the relationship presented in equation 1 implies that the length of the wire directly effects the HOMO and LUMO energy levels, and therefore the band gap. Since electrons of conjugated polymers delocalize through $p_{z}$ orbitals of the main chain, conjugation length is analogous to the theoretical wire length. Therefore, promoting orbital overlap by controlling the backbone morphology of conjugated polymers represents an alternative method by which to influence the band gap energy.

\subsection{Backbone Morphology of Conjugated Polymers}

While chemical doping and the model of a particle on an infinitely thin wire both improve and explain charge transport of conjugated polymers within an individual chain (intra-chain transport), electrons may also move perpendicularly between separate chains (inter-chain transport) ${ }^{21}$. In the ideal case, conjugated polymers may be thought of as perfectly rigid and linear conductive wires, with of intra-chain charge transport relative to inter-chain charge hopping. In this scenario we will assume all conjugated polymer chains have identical degrees of polymerization, and therefore have indistinguishable conjugation lengths. 
However, from an applications perspective this ideal example is ultimately impractical. To work effectively, the length of the individual polymer chains would have to span the entire structure of a device, which has never been achieved. Consequently, macroscopic conductivity over long distances in bulk conjugated polymers is dependent on inter-chain mechanisms. Unfortunately, such mechanisms are greatly hindered by alignment defects along individual polymer main chains ${ }^{22}$. These imperfections introduce charge traps into the conjugated backbone, and suppress two dimensional conductivities. Importantly, these defects may be diminished by the appropriate control of conjugated polymer microstructure and crystallinity, allowing for augmented two dimensional mobility of charge carriers through intra and inter-chain transport.
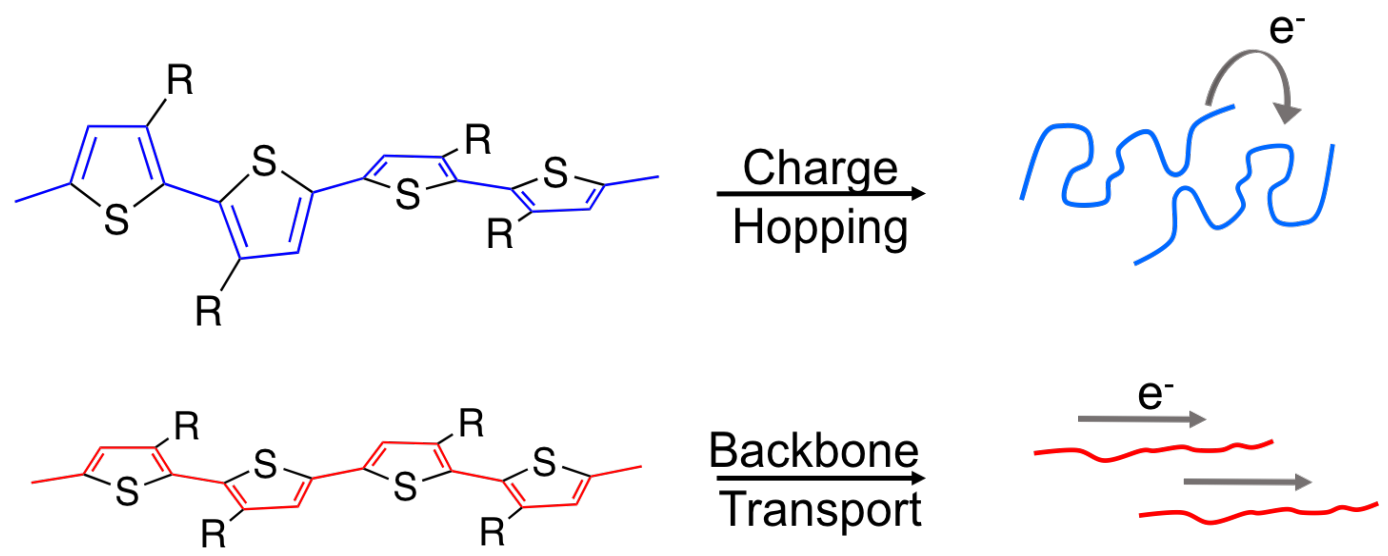

Figure 8. The different directions of 2-D charge transport in organic semiconductors: Intermolecular charge hopping (top) and intramolecular backbone transport (bottom). 
Control of crystalline morphologies in conjugated polymers, namely poly(3hexylthiophene) (P3HT), have been demonstrated to enhance the performance of photovoltaic devices ${ }^{23}$. Kinks and bends along the conjugated polymer backbone act to effectively increase the systems band gap. However, promotion of crystalline regions between backbones leads to more rigid and ordered structures, which correspondingly results in the geometrical alignment of $p_{z}$ orbitals. As discussed previously, the infinitely thin wire model shows that alignment of $p_{z}$ orbitals increases the conjugation length, and reduces the band gap energy. However, as polymers never exist as completely crystalline materials, conjugation is not continuous, but instead exists in rather discrete regions. The ensemble average of such regions has therefore been deemed as the effective conjugation length, and correlates well with overall chain rigidity and crystallinity.

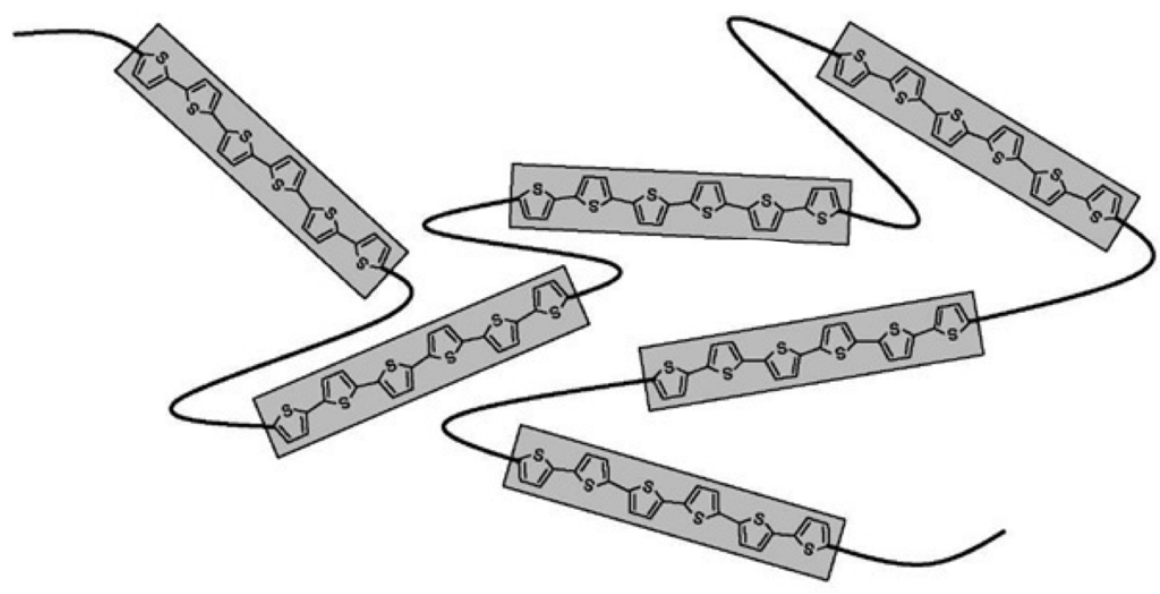

Figure 9. A polythiophene chain with planarized segments (grey). The total length sum of the grey regions is the effective conjugation length of the chain ${ }^{24}$. 
P3ATs are well studied systems due to their reduced band gaps and impressive charge carrier mobility ${ }^{25,26}$. Additionally, P3ATs have large ionization potentials $(\sim 5 \mathrm{eV})$ that prevent oxidation under ambient conditions, imparting stability for photovoltaic device applications ${ }^{27}$. This is in opposition to highly conductive organic counterparts, such as polyacetylene, which oxidizes readily under ambient environments. The most studied of the P3AT family is P3HT, which has an effective conjugation length that is directly related to its backbone orientation and morphology. While numerous factors can affect these orientations, investigations of how solvent choice influences $\mathrm{P} 3 \mathrm{HT}$ crystallinity are ubiquitous throughout the literature ${ }^{28,29}$. In P3HT, solution-based self organization forces the thiophene monomer units to align in anti-coplanar arrangements. These orientations result from solvent interactions with the polymer backbone that eventually lead to an equilibrium conformation. In a good solvent, the polymer backbone is highly solvated, and exists in a stable coil-like conformation. In contrast, poor solvents introduce thermodynamic instability into the system, and backbone conformation is altered to achieve a more stable state. Rod-like conformations are thus promoted due to their lower resonance energy and delocalization tendencies ${ }^{30}$. As such, the rod-like conformation of P3HT enhances the backbone planarity and effective conjugation length. The formation of backbone planarity is commonly accompanied by the presence of rod-like aggregates ${ }^{30}$. Intriguingly, intermolecular forces between polymer chains, especially $\pi-\pi$ stacking interactions between $p_{z}$ orbitals of the thiophene rings, are believed to play a role in these aggregation phenomena ${ }^{31}$. As such, it is these 
interactions that arbitrate solid-state crystal formation. Yet, dried films of P3HT aggregates lack long range order and are inhomogeneous, providing an additional barrier to enhancing conductivity. Furthermore, solvent choice also plays a significant role in coatings processes for roll-to-roll printing. Properties such as solution rheology, film thickness, ambient vapor pressure, and environmental impact must all be considered. Thus a delicate balance between the latter properties, P3HT solubility, and crystal formation should be achieved through careful solvent selection.

\subsection{Mechanisms of Polythiophene Self-Assembly}

From a mechanistic point of view, the relationship between chain alignment and intermolecular aggregation is a chicken or the egg scenario. That is, do individual polymer chains align and subsequently aggregate, or does aggregation force chain planarization? In the latter case, intermolecular aggregation of multiple chain segments is a single step mechanism that leads to fringed micelle-like structures $^{32}$. As the chains come together, forced planarization of the polythiophene backbone is induced. In contrast, the latter mechanism calls for individual polythiophene chains to undergo intramolecular reorganizations from coil-like to rod-like conformations ${ }^{32}$. These rod-like chain segments subsequently aggregate following backbone reorganization. As such, the intramolecular coil-torod mechanism consists of two steps: (1) intramolecular reorganization followed by (2) intermolecular aggregation ${ }^{33}$. The intramolecular vs. intermolecular origin of 
conjugated polymer self-organizations has been heavily debated for multiple systems, including polydiacetylenes and polythiophenes.

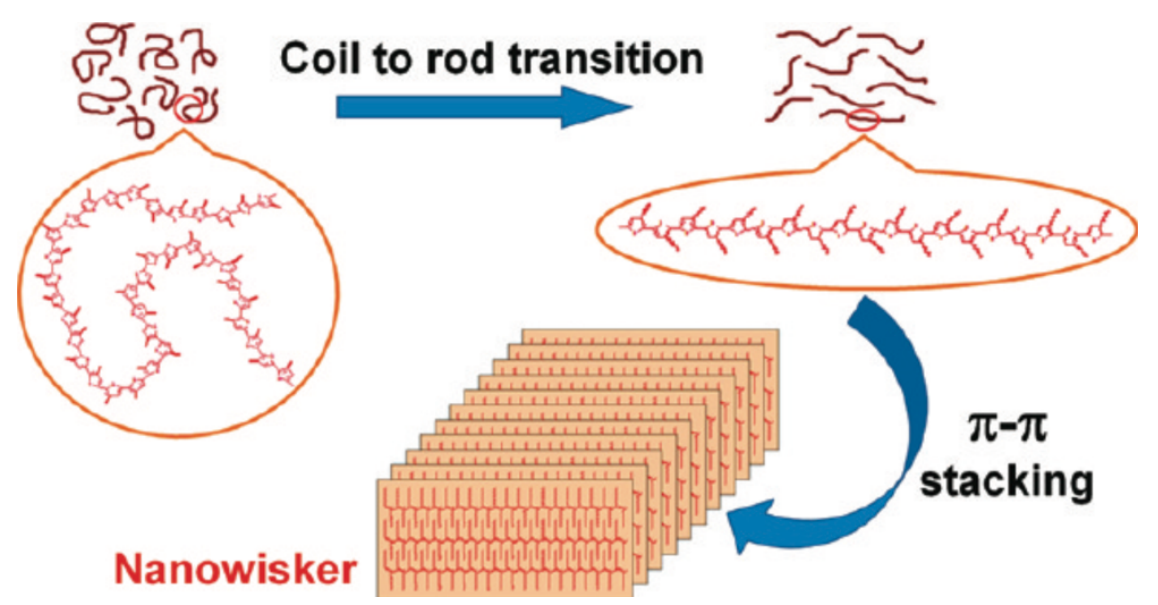

Figure 10. Two step mechanism of the coil-to-rod transition in poly(3alkylthiophenes) $)^{30}$.

It is understood that the self-organization mechanism of P3AT assembly results from a coil-to-rod conformational transition, which may be of inter- or intamolecular origin to form aggregates of nanofibrils. Structurally, such nanofibrils are upheld through the formation of hairpin-like lamella that propagate through the back and forth folding of polymer chains. As mentioned previously, some believe the coil-to-rod transition to be the primary driver for self-assembly, while others insist rod-rod aggregation dominates. Previous reports have shown the solution assembly kinetics of $\mathrm{P} 3 \mathrm{HT}$ in a marginal solvent to be of a mixed fractional order, with an exponent of approximately $1.6^{34,35}$. Such results may be explained through the secondary nucleation theory, which states that the growth rate $(G)$ of assembly is proportional to the total nucleation (S), such that $G \propto S$. Consequently, it may be 
possible to explain the growth rates of numerous P3AT systems by the total individual contributions of nucleation $\left(\mathrm{S}_{\mathrm{i}}\right)$ from various physical processes ${ }^{35}$. It can then be stated that the total nucleation of P3ATs will result from differing influences of the following components in equation 2

$$
S_{\text {total }}=\sum W_{i} S_{i}=W_{\text {coil }} S_{\text {coil }}+W_{\text {fold }} S_{\text {fold }}+W_{\text {aggreg }} S_{\text {aggreg }}
$$

where $\mathrm{W}_{\mathrm{i}}$ is the weighting factor determining the intrinsic contribution of each term. Additionally, each contributor has a fundamental scaling relationship associated with the physical process it represents. For instance, the chain folding process has previously revealed kinetics of a fractional order, similar to those reported for $\mathrm{P} 3 \mathrm{HT}$ in a marginal solvent. However, controlling the weighting factors associated with the coil-to-rod and aggregation processes has yet to be explained unambiguously for polythiophene systems. Moreover, control of such self-assembly mechanisms may allow for the optimization of solid-state morphologies and processing conditions.

\subsection{Solution Processing of Conjugated Polymers}

Liquid crystals (LC) represent a specific phase of matter that lies at the nexus of solids and liquids. As the name implies, LCs maintain an overall ordered structure, similar to solids, while simultaneously remaining fluid, like liquids ${ }^{36}$. The formation of the liquid crystalline state may be a function of either solution concentration (lyotopic LC) or temperature (thermotropic LC). With respect to 
conjugated polymers, lyotropic processing has become an immense interest as it possesses the potential to produce macroscopically ordered thin films.

Crystalline Solid

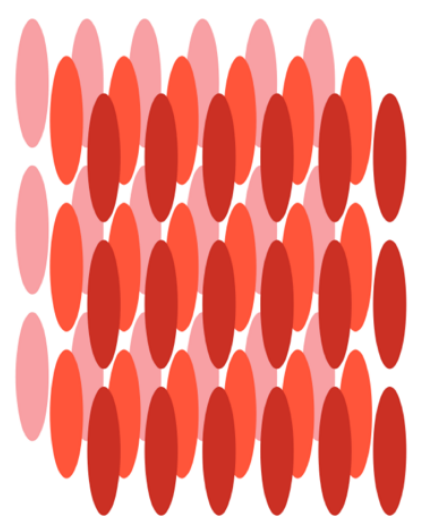

Liquid Crystal

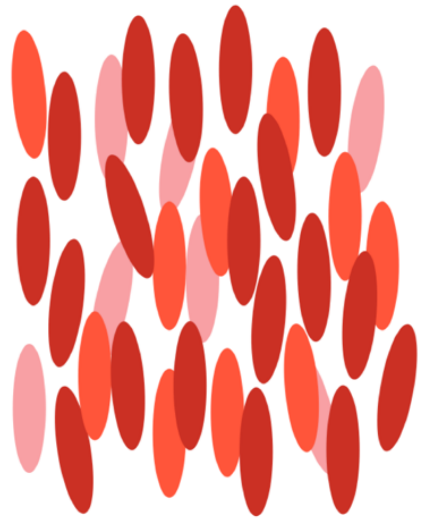

Isotropic Liquid

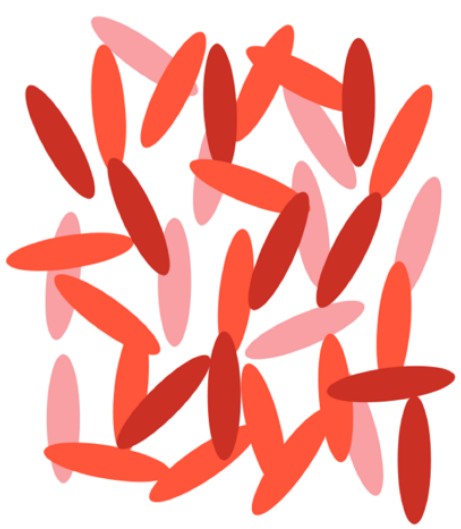

Figure 11. Structural and morphological differences between crystalline solid, liquid crystalline, and isotropic states ${ }^{37}$.

Lyotropic LCs were first investigated in aqueous systems of surfactant molecules ${ }^{36}$. Above the critical micelle concentration (CMC), self-organization of free surfactants into organized micelles becomes thermodynamically favorable. As concentration increases, the surfactant molecules pack into various shapes, including cubes, hexagons, and lamella. The produced shapes then go on to form ordered domains of lyotropic LCs. 


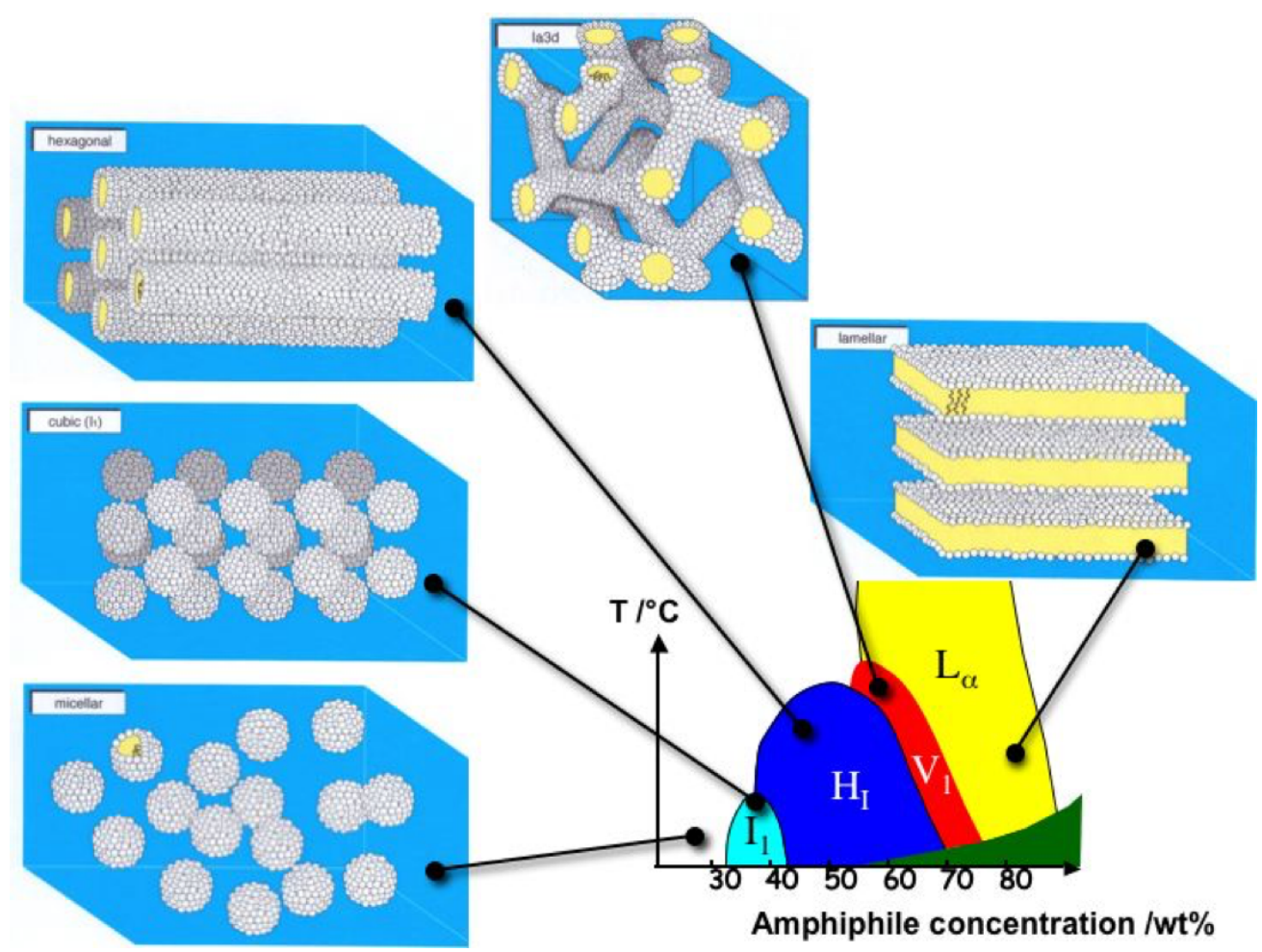

Figure 12. Characteristic phase diagrams of aqueous lyotropic surfactant molecules. Below an appropriate temperature solid state crystals begin to form ${ }^{38}$.

It was not until scientists at DuPont formed lyotropic LCs of the well known polyaramid, Kevlar, that solution processing of polymeric materials began gaining momentum. By using a combination of intermolecular hydrogen bonding and shear-induced alignment, films and fibers produced from Kevlar yielded exceedingly high tensile strengths ${ }^{39}$. Since that time, it has been realized that the long range order inherent in LC materials may be promoted to the solid state. In the case of conjugated polymers, solution-based self-assembly of lyotropic LCs has been shown to produce thin films with an increase in conductivity by three orders of magnitude in the direction of aligned polymer chains relative to perpendicular orientations ${ }^{40}$. As such, the use of conjugated polymer LCs has 
shown promise in improving optoelectronic device effeciencies ${ }^{41-43}$. On the basis of Onsager's rigid-rod model, the promotion of smectic and nematic LC phases is driven through the presence of rigid, high aspect ratio microstructures ${ }^{44}$. As the concentration of a rod-like particle solution increases, a rotational component of entropy is minimized. To counteract this effect, adjacent rod-like particles pack more efficiently to exclude solvent volume, increasing the overall entropy of the system and forming the LC phase. In this way, ordered LC domains that span over hundreds of micrometers may be promoted in thin-films constructed from dilute rod-like particle solutions. Moreover, alignment of LC domains that span even greater length scales may be achieved through external influences, such as mechanical shearing (as was the case with Kevlar), electric, or magnetics fields ${ }^{40,45}$.

(a)

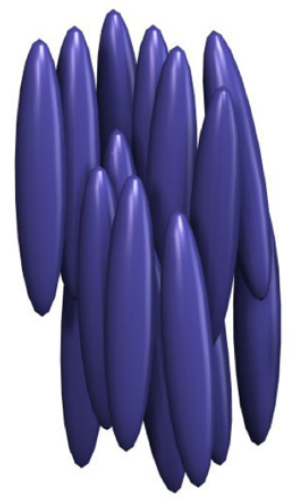

(b)

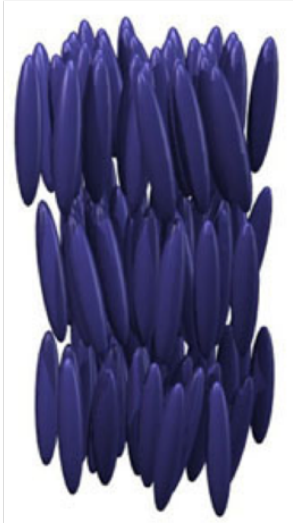

(c)

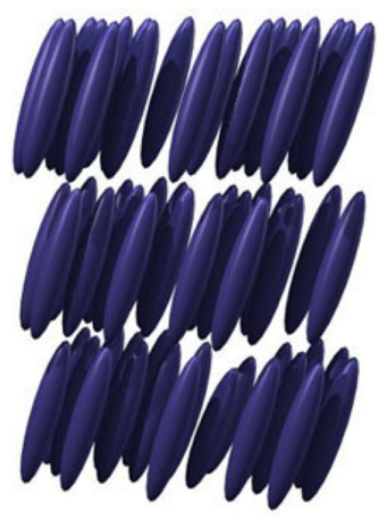

Figure 13. Rod-like particles organized into (a) nematic, (b) smectic A, and (c) smectic C LC phases ${ }^{46}$. 
In addition to achieving long range order in conjugated polymer thin films, the presence of an interconnected network structure is highly desirable for photovoltaic applications ${ }^{47,48}$. However, this goal is difficult to achieve when the processing conditions of conjugated systems highly influence structural development. Because of this, self-assembly prior to film deposition can help ensure the formation of a desirable three dimensional network structure. Such a configuration can be obtained through the formation of a gel, which is a material with subunits that bond to form a network. Bonds linking the subunits can either be formed through covalent means, indicating the gel is permanent, or through intermolecular interaction, leading to thermoreversibility. Importantly, gelation of conjugated polymers in organic solvents is a common phenomenon, and the formation of P3AT organogel systems have been widely reported in the literature ${ }^{49}$. Interestingly, polymer entanglement helps to foster the formation of crystalline nanofibrils, which can then promote the construction of an interconnected 3-D network.

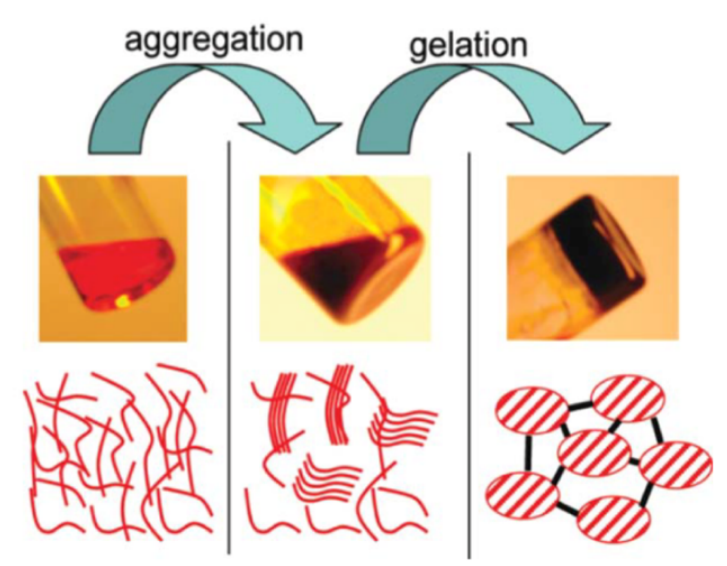

Figure 14. Photos and a schematic illustration of a two-step gelation process of P3HT dissolved in o-xylene ${ }^{50}$. 
Malik et al. demonstrated that interconnected fiber networks present in P3HT gels led to higher charge mobility compared to traditionally produced films from spincasting $^{51}$. While the elastic nature of gels makes them difficult to process through many traditional coatings techniques, recent efforts have demonstrated the feasibility of using conjugated polymer organogels in the processing of photovoltaic devices, including the formation of microgel particles, freeze-drying methods, and organogel sonication ${ }^{47}$. Additionally, non-halogenated/low volatility solvents have been used to control P3AT gelation while allowing for the formation of uniform thinfilm gels under ambient conditions ${ }^{52}$.

While the solution processing of conjugated polymer thin films provides strict advantages in the production of optoelectronic devices over costly inorganicbased counterparts, it also introduces its own set of concerns. The use of halogenated solvents, including chloroform, chlorobenzene, and dichlorobenzene are often utilized to mitigate issues observed in the film forming process of conjugated polymers ${ }^{53}$. However, solvents such as these are harmful to human health and yield a high energy cost due to their toxicity and environmental impact ${ }^{54}$. Such properties lend these solvents to being unsustainable, and stricter regulations on the mass production of these chemicals only makes the processing of conjugated polymers less promising. As such, further efforts have been focused towards achieving environmentally friendly methodologies for the processing of conjugated polymer thin films, and have been successful in producing photovoltaic cells with $7 \%$ power conversion efficiencies ${ }^{55,56}$. Until recently, significant efforts were not put forth to produce optoelectronic devices through environmentally 
friendly means. This is most likely a result of the delicate interplay between polymer morphology, solvent composition, and photovoltaic device performance. Therefore, any change in the polymer nanomorphology will result in a change in device performance. This means new understandings between the interplay of nanomorphology and device performance must be established for conjugated polymer systems consisting of benign solvents and new functionalities.

In order to facilitate the processing of conjugated polymers in more polar solvent systems, modifications to structure and side-chain functionality have been synthetically employed. This typically calls for the incorporation of charged polar groups along the backbones of conjugated polymers, resulting in so called conjugated polyelectrolytes. The inclusion of such new functionalities along the conjugated polymer backbone also leads to water solubility, providing a probable pathway to environmentally friendly solution processing ${ }^{57}$. However, conjugated polyelectrolyte films constructed from aqueous solutions lack the crystalline characteristics necessary to enhance photovoltaic device performance. This is the result of aggregate formation in aqueous solutions of conjugated polyelectrolytes. The coloumbic repulsion of the ionic side-chains leads to backbone interactions through $\pi-\pi$ stacking, which prevents hydrophobic regions of the polymer chains from interacting with the surrounding aqueous medium ${ }^{58}$. Because of this issue, researchers have focused their attentions on tailoring the morphology of conjugated polyelectrolytes in aqueous media.

Surfactants have been commonly employed as additives in aqueous solutions of conjugated polyelectrolytes to mitigate the above mentioned 
aggregation ${ }^{57,58}$. While both ionic and neutral surfactants have been utilized, the use of counterionic surfactants have been shown to result in the formation of supramolecular complexes. This is due to coulombic attraction between charges of the conjugated polyelectrolyte side-chains and surfactant molecules. Interestingly, the formation of lyotropic liquid crystals have been reported in aqueous solutions of the surfactant centyltrimethylammonium bromide complexed with a conjugated polyelectrolyte derivative of polythiophene in 1:1 molar ratios ${ }^{59}$. Further analysis by small-angle X-ray scattering suggested that the supramolecular complexes formed hexagonal microstructures above a critical solution concentration. The self-assembly of such microstructures may provide a suitable methodology for the control of conjugated polyelectrolyte morphology in aqueous media.

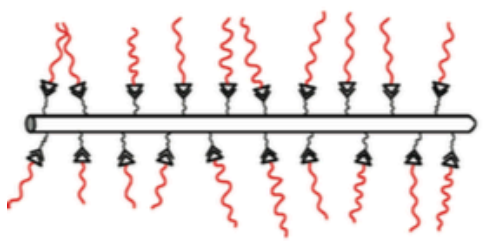

(a)

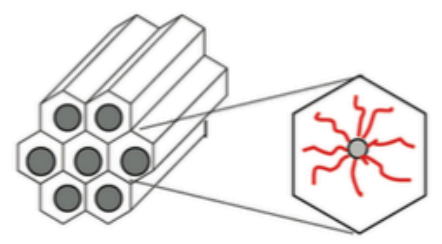

(c)

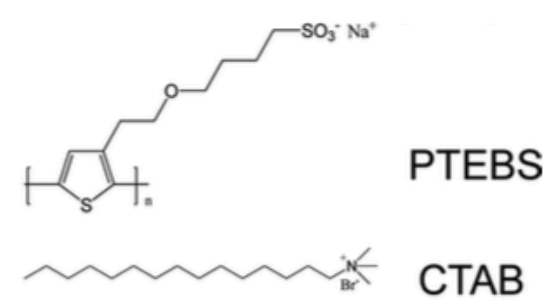

(b)

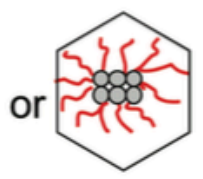

Figure 15. (a) Model of CTAB complexed onto the backbone of PTEBS. (b) Chemical structures of the surfactant (CTAB) and the polymer (PTEBS). (c) Depiction of hexagonal packing in the lyotropic LC phase ${ }^{59}$. 
Aside from the incorporation of electrolytes onto the backbone structure of polythiophenes, functional groups that allow for short-range interactions between thiophene units have also been produced ${ }^{60,61}$. One such common functionality is that of a carboxylic acid, or any other group which has the potential to act as both a hydrogen bond donor and acceptor. In contrast to the repulsive forces of similarly charged side-chains, hydrogen bonding allows for attraction between polymer chains, promoting thermorevesible linking between adjacent monomer units. Such interactions have been found to assist in the processing conditions conjugated polymers, and even result in the formation of gels ${ }^{62}$. These intermolecular cross-links incorporate plasticity into the polymer gel, and allow for ease of alignment processes. As such, the investigation of polythiophene derivatives with the potential to hydrogen bond may provide interesting processing alternatives compares to polythiophene-based CPEs.

\subsection{Multi-Scale Assembly Methodology of Conjugated Polymers}

When using a multi-scale assembly approach, modifications to the conjugated polymer system (surfactant addition, poor-solvent addition, etc.) are made in the dilute regime. Such adjustments are done with the intention of promoting high aspect ratio microstructures through the coil-to-rod transition. By raising concentrations of the rod-like particle solutions past a critical value, the promotion of lyotropic LC phases may be obtained, which yield highly ordered domains of conjugated polymers. Through the application of an external stimulus across still-wet LC samples, uniform monodomains of conjugated polymer thin 
films are produced that sustain significant optoelectronic anisotropy in the solid state.

In this work, enhanced anisotropy of poly(3-hexylthiophene) derivative thin films was achieved for two separate systems using a multi-scale assembly methodology. In the first system, carboxylate functionalized P3HT is ionically bonded with aqueous quaternary ammonium surfactants in 1:1 molar ratios to form a supramolecular charge-neutral complex. For the second system, carboxylic acid functionalized $\mathrm{P} 3 \mathrm{HT}$ is dissolved in various ratios of dimethyl sulfoxide and ethanol. In dilute solutions of systems 1 and 2, the coil-to-rod transition is investigated as a function of surfactant architecture and solvent composition, respectively. The origin of the coil-to-rod mechanism is revealed for both systems through transition kinetics. In concentrated solutions, both systems under-go isotropic to LC phase transitions. Interestingly, system 2 forms an LC gel in contrast to system 1 . The mechanism of the coil-to-rod transition is believed to play a role in the resulting LC gelation. The application of a mechanical shear force aligns the LC samples of both systems, and a significant optoelectronic anisotropy is observed in the dried thin films. 


\section{Experimental Methods}

\subsection{Material Preparation}

\subsubsection{System 1}

Regioregular (82-90\% head-to-tail) poly[3-(potassium- 4butanoatethiophene)-2,5-diyl] (P3K4T, average $\mathrm{Mw}=14 \mathrm{~kg} / \mathrm{mol}$, Rieke Metals Inc.), poly[3-(potassium-5-penanoatethiophene)-2,5- diyl] (P3K5T, average Mw = $35 \mathrm{~kg} / \mathrm{mol}$, Rieke Metals Inc.), poly[3-(potassium-6-hexanoatethiophene)-2,5-diyl] (P3K6T, average $M w=60 \mathrm{~kg} / \mathrm{mol}$, Rieke Metals Inc.), poly[3-(potassium-7heptanoatethio- phene)-2,5-diyl] (P3K7T, average Mw = $60 \mathrm{~kg} / \mathrm{mol}$, Rieke Metals Inc.), centyltrimethylammonium bromide (Sigma-Aldrich) including its various alkyl chain derivatives with the side-chain length equal to 10,12 , and 14 carbons, and dihexadecyl dimethylammonium bromide (Sigma-Aldrich) were used as received in preparation of stock hydrogels $(0.10 \mathrm{M})$. Initially, individual polymer and surfactant solutions were prepared in 1:1 molar ratios at concentrations of $0.20 \mathrm{M}$ in plastic microcentrifuge tubes. All solutions were made with nanopure water (Milli-Q, $18 \mathrm{M} \Omega$ ). After dissolution, individual polymer and surfactant solutions were sonicated for 20 minutes at a temperature of $60^{\circ} \mathrm{C}$. The samples were then heated in a temperature controlled oil bath at $70^{\circ} \mathrm{C}$ for 1 hour or until fluid. While still in the oil bath, solutions were transferred by dropwise addition of surfactant to polymer, resulting in supramolecularly complexed samples with concentrations of $0.10 \mathrm{M}$. The supramolecular complex was then quickly removed from heat and vortexed for $20 \mathrm{~min}$, during which the samples were allowed to gradually cool to room temperature. The supramolecular complexes were then microcentrifuged (18- 
Centrifuge Biotechnical Services Inc.) for $30 \mathrm{~min}$ at a speed of $10,000 \mathrm{rpm}$, resulting in stable hydrogels. Little to no phase separation was noticed after centrifugation. The chemical structures and nomenclature of both polymers and surfactants are shown in Figure 16.

(a)<smiles>CC(C)(C)c1cc(C(C)(C)C)c(C(C)(C)C)s1</smiles>

(d)

(e)

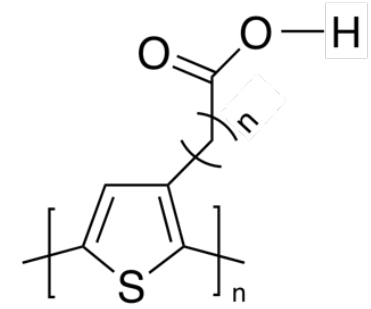

(b)<smiles>CN(C(C)(C)C)[N+](C)(C)Br</smiles>

(c)<smiles>[B]N(C)C(C)(C)N(C)C(C)(C)C</smiles>

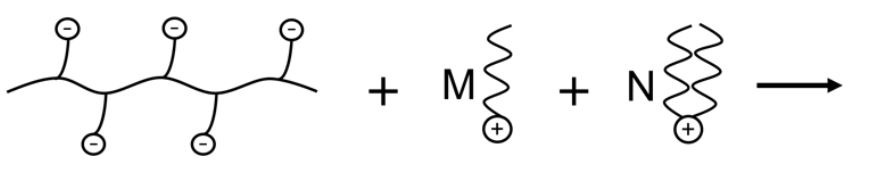

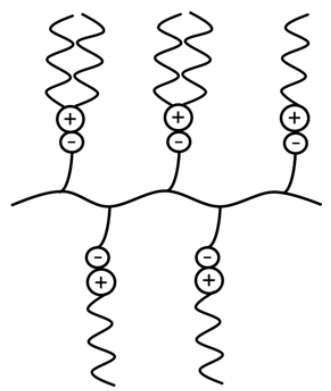

Figure 16. (a) Chemical structure of polythiophene P3KnT (system 1) with $n=4$, 5,6 , or 7 , (b) single-tailed surfactant (STSn) with $n=10,12,14$, or 16 , (c) doubletailed surfactant (DTSn) with $n=16$, (d) illustration of the supramolecular complexation of P3KnT with single and double- tailed surfactants, and (e) chemical structure of polythiophene P3CnT (system 2) with $n=3,4,5$, or 6 . 
For DTS, extended sonication and heating was required due to heightened hydrophobicity of the surfactant molecules. Because of this, DTS was only added onto the P3K6T backbone with STS ${ }_{16}$ at a maximum ratio of 3:7. All concentrations noted for this system are with respect to the monomeric repeat unit. To prepare liquid crystal solutions, sample hydrogels were heated for 24 to 48 hours at $80^{\circ} \mathrm{C}$ to ensure complete evaporation of water from the sample. The dried film was then weighed by difference, and re-dissolved at an appropriate concentration in weight percent. To produce solid films, a small aliquot of liquid crystal solution was placed on a glass slide. A glass coverslip was applied against the slide and the sample was subsequently sheared by hand at a high coatings speed. The application of shear would completely remove the cover slip. The sample would then be left in a desiccator until dry.

\subsubsection{System 2}

Regioregular (90-95\% head-to-tail) Poly[3-(5-carboxypentyl) thiophene-2,5diyl] (P3C5T, average $\mathrm{M}_{\mathrm{W}}=60 \mathrm{~kg} / \mathrm{mol}$, Rieke Metals Inc.), regioregular (90-95\% head-to-tail) Poly[3-(5-carboxyhexyl) thiophene-2,5-diyl] (P3C6T, average $\mathrm{M}_{\mathrm{W}}=$ 55 kg/mol, Rieke Metals Inc.), regioregular (90-95\% head-to-tail) Poly[3-(5carboxybutyl) thiophene-2,5-diyl] (P3C4T, average $\mathrm{M}_{\mathrm{w}}=35 \mathrm{~kg} / \mathrm{mol}$, Rieke Metals Inc.), regioregular (90-95\% head-to-tail) Poly[3-(5-carboxypropyl) thiophene-2,5diyl] (P3C3T, average $M_{W}=20 \mathrm{~kg} / \mathrm{mol}$, Rieke Metals Inc.), dimethyl sulfoxide (DMSO, Fisher Scientific), and ethanol (EtOH, BDH 95\% reagent grade) were

used as received in preparation of stock solutions $(2.5 \mathrm{mg} / \mathrm{mL}$, unless otherwise 
stated). The generalized chemical structure of all polymers (P3CnTs) may be seen in Figure 16e. All samples of the P3CnT stock solutions were first prepared in DMSO. The samples were then heated to $80^{\circ} \mathrm{C}$ until the solution exhibited a bright orange color. For dilute samples containing a mixture of DMSO and $\mathrm{EtOH}$ solvents, the stock solution was diluted to an appropriate concentration in DMSO. The dilute sample was then heated until it displayed a bright orange color, indicating complete dissolution. The sample was then removed from the heat source, and an appropriate amount of $\mathrm{EtOH}$ was added drop-wise to the solution.

When preparing more concentrated samples, a small amount of the appropriate solvent would be added to the polymer sample. The sample would then be heated overnight at $80^{\circ} \mathrm{C}$ in a sealed glass vial to ensure homogeneity. To prepare LC textured samples, a small aliquot of the concentrated solution was cast onto a clean glass slide. A cover slip was then applied atop the sample with $1 \mathrm{~N}$ of force. To induce polymer alignment, a small aliquot of the LC solution was cast onto a clean glass slide. A cover slip was then applied on top of the sample with minimal pressure. The glass slide was then placed in a Linkam LTS420 hot-stage. The hot-stage was programmed using a Linksys 32 software to control the temperature. The sample was then heated to $80^{\circ} \mathrm{C}$ at a rate of $5^{\circ} \mathrm{C} / \mathrm{min}$, or until a color change from dark purple to orange was noted. The sample was left to equilibrate under these conditions for 10 minutes. Cooling of the sample to room temperature was undergone at a rate of $5^{\circ} \mathrm{C} / \mathrm{min}$. A color change from orange to dark purple was observed, and the sample was then quickly removed from the hot stage and rapidly sheared by hand at a high coatings speed. Shearing did not 
result in removal of the coverslip, and the slip was carefully removed with tweezers following exposure to ambient conditions for 1 hour. The sample was then left to air dry for multiple days.

\subsection{Instrumental Methods of Characterization}

\subsubsection{UV-Vis Spectroscopy and Spectrofluorimetry}

All electronic spectra were obtained on a Cary-Win UV-vis spectrophotometer and a Jasco FP-6500 spectrofluorometer. Both instruments contained temperature control fittings set at $25^{\circ} \mathrm{C}$ unless otherwise stated, and all samples were sealed from ambient atmosphere during data collection. Figure 17 displays the working mechanisms of UV-vis and fluorescence spectrometers, respectively.

(a)

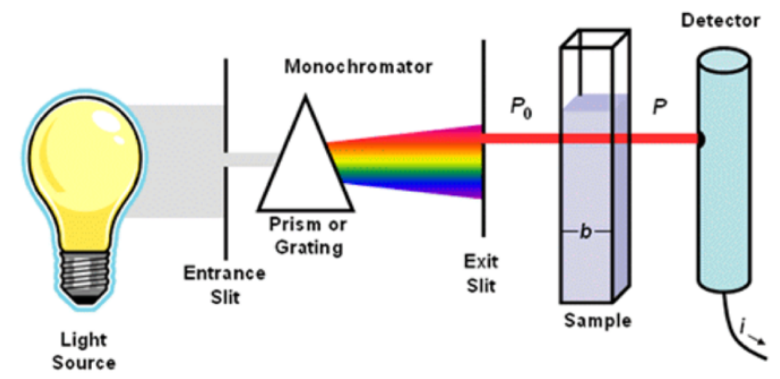

(b)

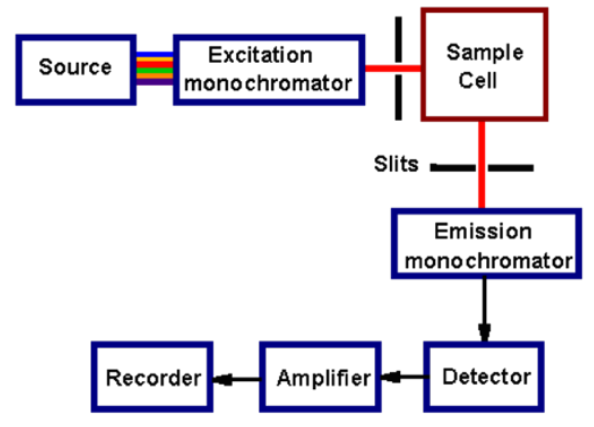

Figure 17. (a) The working mechanism of a UV-vis spectrophotometer. Light passes through an entrance slit and monochromator. Upon leaving the exit slit, monochromatic light passes through the sample and is transmitted to a detector. (b) Working mechanism of a spectrofluorimeter ${ }^{63,64}$. 
For system 1, the viscous nature of the supramolecular hydrogel samples makes determining the concentration of serial dilutions dubious. As such, a calibration curve was constructed for all samples. A stock hydrogel $(0.10 \mathrm{M})$ was first oven-evaporated, and the mass of the supramolecular complex was determined through gravimetry. The sample was then re-dissolved, and serial dilutions were made at concentrations of $0.10,0.15,0.20,0.25,0.375$ and 0.50 $\mathrm{mM}$. Spectroscopic measurements were taken at a temperature of $50^{\circ} \mathrm{C}$ with quartz curvettes. This ensures consistency between the relative wavelength maximums of each diluted sample. The absorbance of an analyte can then be related to its concentrations using equation 3 (Beer's Law)

$$
A=\varepsilon b c
$$

where $A$ is the absorbance, $\varepsilon$ is the molar absorptivity, $b$ is the pathlength $(1 \mathrm{~cm}$ for the quartz cuvette), and $\mathrm{c}$ is the concentration of the analyte. By plotting the the absorbance at the wavelength maximum of each sample against its respective concentration, a linear plot is produced. By applying a linear regression model, the Beer's law relationship is thus experimentally derived, and the concentration of any sample within the range of the calibration curve may be determined. An example of such a calibration curve is demonstrated in Figure 18. For kinetics measurements, the sample was heated to $60^{\circ} \mathrm{C}$ and then diluted into a temperature controlled cuvette containing water. This method allowed for kinetics measurements to begin as quickly as possible, and would simultaneously quench 
the sample to room temperature, thereby imparting isothermal conditions. The absorbance vs. time data was collected for a wavelength of $590 \mathrm{~nm}$ over the course of 3 minutes. The initial rate was determined from the first 15 to 30 seconds of data collection. Linear fits of the initial rates all demonstrated $R^{2}$ values greater than $90 \%$.

(a)

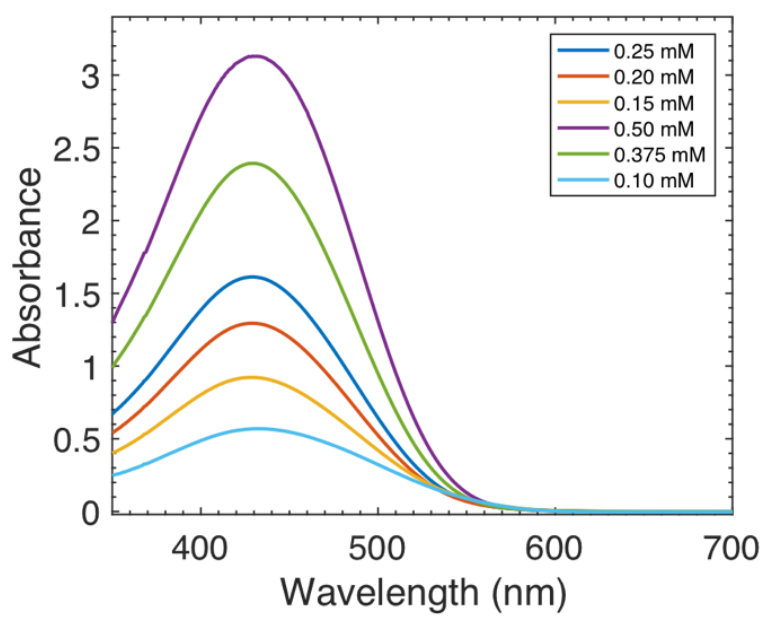

(b)

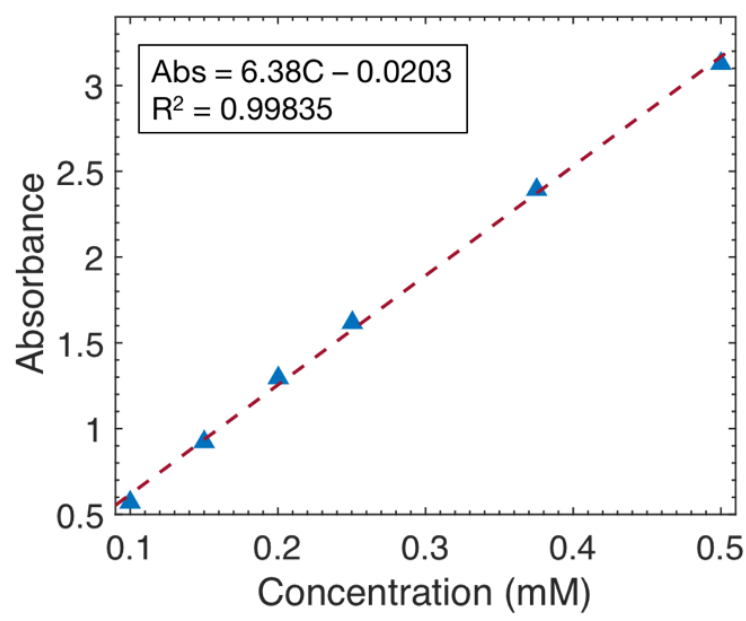

Figure 18. (a) Concentration dependent UV-vis spectra of the 1:1 P3K6T:STS ${ }_{16}$ supramolecular complex at $50^{\circ} \mathrm{C}$. (b) Calibration curve of the $1: 1 \mathrm{P} 3 \mathrm{~K} 6 \mathrm{~T}: \mathrm{STS} \mathrm{S}_{16}$ supramolecular complex.

For system 2, spectroscopic measurements of dilute solutions were obtained on a Cary-Win UV-vis spectrophotometer. The temperature controller was set to $25^{\circ} \mathrm{C}$ and the samples were closed from ambient air atmosphere. Typically, samples were heated to $70^{\circ} \mathrm{C}$ in a temperature controlled water bath just prior to spectroscopy measurements. The samples were then quickly transferred and further diluted into a cuvette, which was preset at $25^{\circ} \mathrm{C}$. As stock solutions of this system did not form a hydrogel, the construction of calibration curves was not 
necessary. For temperature dependent measurements, a cuvette was filled with 2 $\mathrm{mL}$ of sample at an appropriate concentration. The cuvette was then placed in a sample cell at $20^{\circ} \mathrm{C}$ and left to equilibrate for 15 minutes. Samples were then run at various temperatures after 15 minutes of equilibration between measurements.

\subsubsection{Small-Angle X-Ray Scattering}

Small-angle X-ray scattering (SAXS) measurements were only performed for system 1. In this case, SAXS was utilized for both dilute solution measurements as well as concentrated LC solutions. As the California Polytechnic State University in San Luis Obispo does not possess SAXS instrumentation, a collaboration between South Carolina and Yale Universities was established. For dilute solution measurements, all samples were run in the lab of Dr. Chinedum Osujii in the Department of Chemical and Environmental Engineering at Yale University. Dilute solutions of the supramolecular complexes were prepared at various concentrations under $1 \% \mathrm{w} / \mathrm{w}$ total solids. Theoretically, this concentration was expected to be below the overlap concentration for completely rigid chains, assuming a repeat unit length of $0.446 \mathrm{~nm}^{65}$. SAXS measurements were performed on a pinhole collimated Rigaku instrument (SMAX3000) with a $1.54 \AA$ $\mathrm{Cu} \mathrm{Ka}$ radiation source. The scattering data was recorded using a 2D multiwire electronic area gas detector. The instrument was calibrated using silver behenate with a d-spacing of $58.38 \AA$. Scattering patterns were obtained over a period of 5 h of X-ray exposure. The samples were sealed in Kapton capillary tubes with an 
acrylic adhesive, and were heated in situ by a custom-built temperature controller to within $0.1^{\circ} \mathrm{C}$.

For concentrated LC solutions, samples were shipped to Dr. Morgan Stefik's lab in the Department of Chemistry and Biochemistry at South Carolina University. For all samples, SAXS experiments were conducted using a Linkam Scientific Instrument HFS350X-GI hot stage in a SAXSLab Ganesha. A Xenocs GeniX3D microfocus source was used with a Cu target to generate a monochromic beam with a $1.54 \AA$ wavelength. Calibration was performed using National Institute of Standard and Technology (NIST) reference material, 640c silicon powder with a peak position at $2 \theta=28.44^{\circ}$, where $2 \theta$ is the total scattering angle. A Pilatus 300 $\mathrm{K}$ detector (Dectris) was used to collect the two- dimensional (2D) scattering patterns. 2D images were azimuthally integrated to one-dimensional (1D) data of intensity (I) versus scattering vector q. The samples were placed between mica or Kapton windows (mica thickness of mica $\sim 20 \mu \mathrm{m}$, from Molmex Scientific Inc.) with a rubber O-ring positioned carefully. All data were acquired after equilibration for $30 \mathrm{~min}$ at each temperature followed by a $1.0 \mathrm{~h}$ measurement with an X-ray flux of $\sim 21.4 \mathrm{M}$ photons/s incident upon the sample.

SAXS is an incredibly informative technique that can provide information into the 3-D structural morphology and anisotropic nature of macromolecules in both dilute solutions and the solid state, respectively. As such, SAXS data provides information about the spatially averaged structural features of macromolecules. The working mechanism of a SAXS instrument may be seen in Figure 19. 


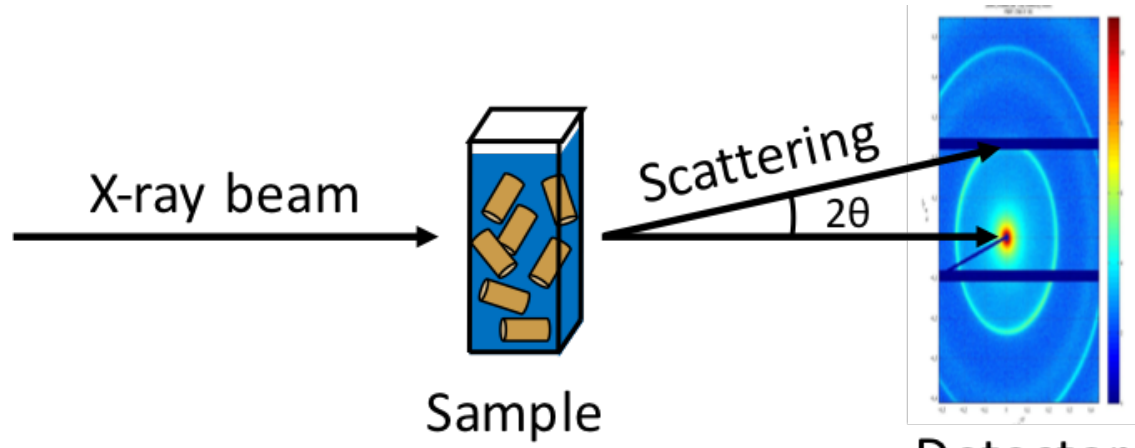

Detector

Figure 19. Transmission geometry SAXS. An imaginary vector connecting the ends of the collimated and scattered X-ray beams represents the momentum transfer between incident and scattered light.

A monochromatic X-ray beam is first fired at the sample. Elastic phenomena between the sample and the incident light causes the photons to scatter. This scattering is then detected by a photodiode beamstop at the end of the instrument. The positioning of the scattered photons is recorded by the photodiode in 2-D, producing what is known as a scattering pattern. In the presence of anisotropic structural characteristics, a greater number of detected photons will appear at specific angles. Through radial integration of the scattering pattern, a plot of scattering intensity against $2 \theta$ is produced. Through equation 4 (Bragg's law) the angle at which scattered peaks are present may be related to the lattice spacing of the material.

$$
n \lambda=2 d \sin \theta
$$


where $\mathrm{n}$ is a whole number integer, $\lambda$ is the wavelength of incident light, $d$ is the spacing of the crystal lattice, and theta is the angle of incoming light. Additionally, further information may be extracted from dilute solution X-ray scattering using the methods of Gunier, Porod, and Gunther, details of which are explained in the results section.

\subsubsection{Polarized Optical Microscopy}

Aside from X-ray diffraction techniques, polarized optical microscopy (POM) is a relatively simple and fast method by which to glean structural information regarding LC textures and solid state morphology. A schlierien texture of the nematic phase under POM is presented in Figure 20a. All LC samples, for both systems 1 and 2, were analyzed using a Leica DM2500P polarized optical microscope. A Leica ICC50 HD video camera was used to capture the images of concentrated LC solutions and gels. The working principles regarding POM are illustrated in Figure 20b.

(a)

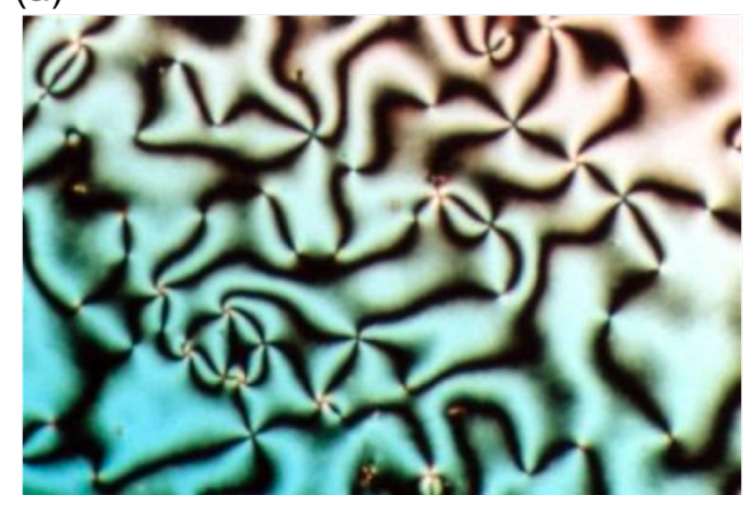

(b)

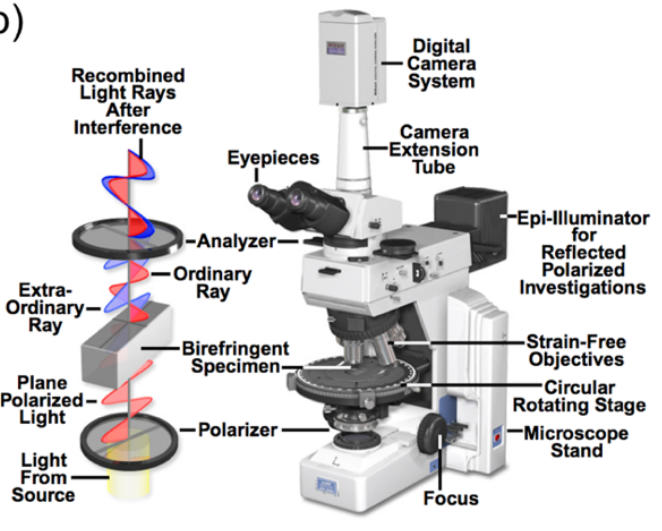

Figure 20. (a) Optical image displaying a schlerien texture of the nematic LC phase. (b) Working mechanism behind $\mathrm{POM}^{46,66}$. 
POM is commonly employed with regards to LC materials in order to determine the LC texture. This can provide information concerning the underlying structure of bulk molecules in the sample. Light is first passed through a polarizer and then the sample. After light exits the sample it then passes through another polarizer (analyzer) at $90^{\circ}$ relative to the first. The light passing through the analyzer is what the user observes through the microscope. Therefore, with an empty sample stage any incident light is eliminated. Placement of a birefringent sample between the polarizers re-polarizes the light, allowing light to pass through the analyzer and produce an image.

POM operates through enhancing the contrast inherent in birefringent specimens. Birefringence is a property characterized by the presence of dual refractive indices in a material. This leads to such a sample producing two individual wave components, each with varying velocities being polarized in perpendicular planes. The two components are referred to as the ordinary and extraordinary wavefronts, and their variation is a function of their propagation within a material. Upon exiting a sample, the two wavefronts are phase shifted and recombine, resulting in constructive and deconstructive interference. Thus, isotropic materials will result in a completely dark image under a POM. Importantly, birefringence is a property observed in highly anisotropic specimens, and POM is therefore an ideal technique for determining the degree of order in LC samples. As all LC solutions and solid state films were prepared on glass substrates, analysis by POM was particularly convenient. 


\subsubsection{Fourier-Transform Infrared Spectroscopy}

Fourier transform infrared (FTIR) spectra were gathered on a Nicolet iS10 FTIR spectrometer with an attenuated total reflection (ATR) mode at a resolution of $4 \mathrm{~cm}^{-1}$ of 1000 scans. FTIR spectra were collected for concentrated LC solutions, gels, and solid films of both systems 1 and 2. For system 1, stock samples of supramolecular hydrogels were prepared by drop-casting onto clean glass substrates. The samples were then oven dried over night in preparation for FTIR measurements. For system 2, concentrated LC gels were prepared and dropcast onto the FTIR stage. A small Styrofoam cup was placed over the sample stage. A needle was used to puncture the cup, and a nitrogen purge line was separately run through a syringe into the Styrofoam chamber for 15 minutes prior to data collection.

\subsubsection{Four-Point Probe}

Conductivity measurements of dried films for both systems 1 and 2 were performed on a Keithley Instrument 2400 SourceMeter in a four-point probe configuration. Measurements were taken with probes both parallel and perpendicular to the direction of shear applied along each sample. Voltage sweeps were run in triplicate for each probe orientation, and the data from each sweep was generated on a scatter plot. A line of best fit was produced for each scatterplot. Film thickness and width measurements were conducted using a Starrett No. 734 micrometer and standard ruler, respectively. For a sample of finite width and non- 
negligible thickness, such as the those produced in this study, equation 5 may be used to calculate the bulk resistivity

$$
\rho=4.53 t\left(\frac{V}{I}\right) f_{1} f_{2}
$$

where $t$ is the sample thickness, $V / I$ is the inverse slope of the experimentally determined I-V curves, and $f_{1}$ and $f_{2}$ are thickness and width corrections factors, respectively ${ }^{67}$. The correction factors are dependent on sample geometry (e.g. is the sample a thin circular slice or a thin rectangular slice?) and the ratios between probe spacing, sample thickness, and sample width. Depending on these parameter, the appropriate correction factors were utilized ${ }^{68}$.

For an extended period of time, resistivity, sheet resistivity, surface resistivity, or bulk resistivity were measured as a quantity irrespective of sample dimensions. However, it is now understood that resistivity of a sample is highly dependent on the materials shape and dimensionality. It was not until 1958 that Smits defined a four-point probe method for accurately measuring sheet resistivity ${ }^{69}$. While developing correction factors for measuring the sheet resistivity of 2-D and circular samples, it was found that the four-probe method was not only useful in measuring surface electronics, but also the bulk electronics of thin films. Since that time, the four-probe method has become an industry standard for measuring the resistivity of semi-conductors.

Typically, a digital dual probe multi-meter can measure resistance values accurately above $1 \Omega$. However, for resistance values below $1 \Omega$ accuracy is 
diminished due to contributions from the lead wires inherent probe resistance. Various contributions to the overall resistance can have an effect when measuring low resistance values, including probe resistance, probe-sample contact resistance, spreading resistance of current flow, and the overall sheet resistance of the semi-conductor ${ }^{67}$. As such, these contributions to the overall resistance must be accounted for to determine overall bulk resistance of a semi-conducting sample. By using four probes, as seen in Figure 21, two outer probes may supply a current through the material, and two inner probes may measure the potential difference produced.

(a)

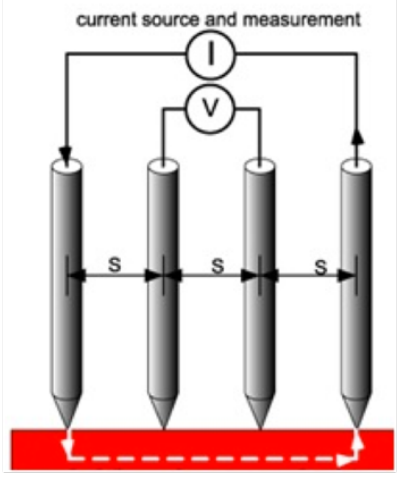

(b)

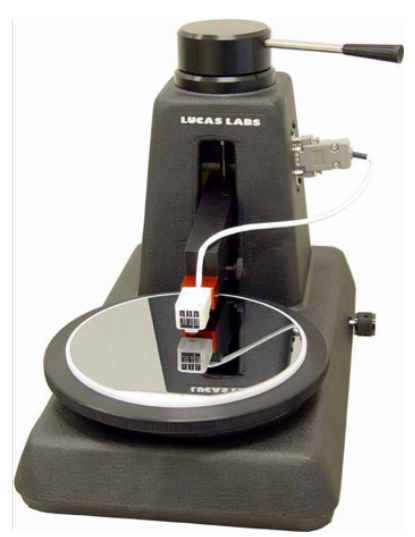

Figure 21. (a) Schematic of a four-probe configuration. Current is supplied through the outer probes, and potential is measured by the inner probes. (b) Image of a four-point probe sample stage ${ }^{70}$.

In this case, the probe spacing (s) is held constant at $0.1026 \mathrm{~cm}$. By using a voltmeter with a large input resistance, the potential difference of the two inner probes is measured and a small current is supplied. Additionally, the opposing currents supplied to the inner probes cancel each other, and the identical 
parameters and dimensions of the probes allows the contributions of various resistivity's to be equivalent. Therefore, the application of Kirchhoff's voltage law to the closed loop of the two inner probes results in only sheet resistance contributing to the overall thin film resistivity. 


\section{Results and Discussion}

\subsection{System 1}

Shifting of optical properties can be induced in CPE's through their exposure to environmental stimuli, offering potential methods for control and analysis of solution-based conformations. These stimuli can be numerous and include changes in concentration, temperature, solvent, $\mathrm{pH}$, or binding of other molecules to the ionic moities ${ }^{58}$. Surfactants have been shown to induce the aforementioned optical shifts in CPE's through complexation onto the ionic sidechains $^{59,71}$. It is understood that upon addition of CPE's to a polar solvent, the resulting polymer chains exist in mildly packed aggregates due to their amphiphilic nature. The ionic side-chains face outwards toward the water-polymer interface, while the polymer backbone interacts inwards through orbital overlap via $\pi-\pi$ stacking ${ }^{58}$. The addition of surfactant hinders $\pi-\pi$ stacking interactions and disrupts aggregation, resulting in blue-shifts of absorption spectra ${ }^{59,72}$. This alteration of optoelectronic properties has been deemed, "surfactochromism" and is dependent on surfactant concentration, molar ratios of surfactant relative to CPE's ${ }^{73,74}$, and surfactant lipophile size. Additionally, P3AT morphology has been shown to depend on alkyl-chain characteristics, including bulky sterics and length ${ }^{75,76,77}$. Even more intriguing, the promotion or hindrance of these characteristics may yield augmented order and conjugation. 


\subsubsection{Effect of Side-Chain Architecture and Length}

To glean further insight into the effects of surfactants on the structural conformations of dilute-solution polythiophene-based CPE's (P3KnT's), differing alkyl-chain lengths of aqueous P3KnT's and single-tailed quaternary ammonium surfactants $\left(\mathrm{STS}_{\mathrm{n}+1}\right)$ were mixed together in 1:1 molar ratios to form a supramolecular complex. Formation of the supramolecular complex was confirmed by FTIR spectroscopy, and spectra were generated for P3KnT's before and after supramolecular complexation with $\mathrm{STS}_{\mathrm{n}-1}$ and $\mathrm{DTS}_{\mathrm{n}-1}$. An example spectrum of 1:1 $\mathrm{P} 3 \mathrm{~K} 6 \mathrm{~T}: \mathrm{STS}_{16}$ is shown below in Figure 22. Alterations in the vibrational energies of methylene bending bands $\left(2800-3000 \mathrm{~cm}^{-1}\right)$ as well as carboxylate stretching bands $\left(1300-1600 \mathrm{~cm}^{-1}\right)$ suggests completion of the complexation reaction. The presence of three peaks in the carboxyl region after supramolecular complexation is indicative of bonded carboxy groups as opposed to free carboxylate ${ }^{78}$. Slight blue shifts in methylene vibrations and sizable reductions of peak half-widths are ascribed to the enhanced packing of aliphatic chains in the supramolecular complexed films. 


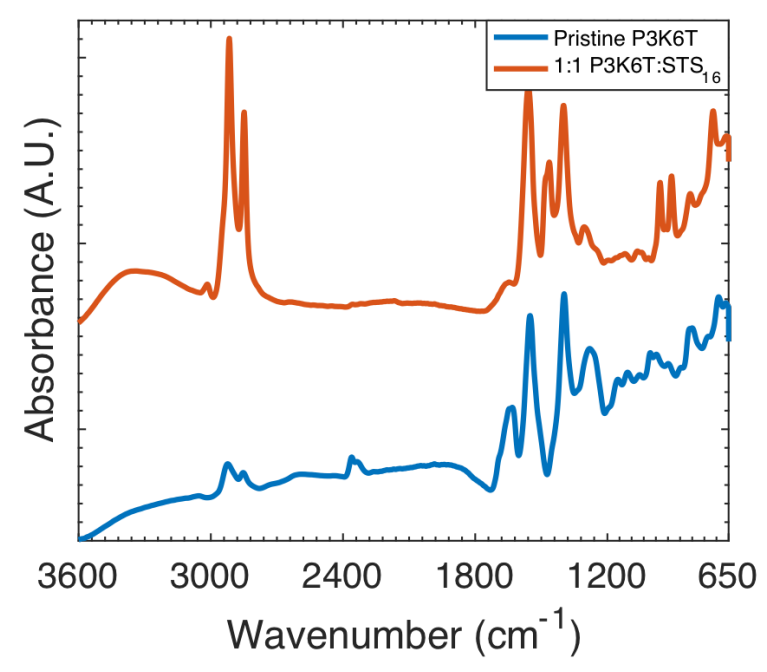

Figure 22. FTIR spectra of pristine P3K6T and 1:1 P3K6T:STS ${ }_{16}$ supramolecular complex.

Figure 23a depicts the absorbance spectrum of pure P3KnT's, while Figure $23 \mathrm{~b}$ shows alterations of optoelectronic properties after complexation with $\mathrm{STS}_{16}$. The UV-Vis spectrum of pure P3KnT's shows substantial red-shifts for P3K5T, P3K6T, and P3K7T. These bathochromic shifts are ascribed to aggregation of polythiophene backbones through $\pi-\pi$ stacking. Furthermore, P3K4T exhibits a broad peak width and a much lower $\lambda_{\max }$ around $420 \mathrm{~nm}$ compared to its longer alkyl-chain counterparts. Previous literature has supported the ability of P3K4T to refrain from complete aggregation in favor of forming small aggregate domains, explaining the coupled blue-shift and peak broadness ${ }^{79}$. Following complexation with surfactant, P3K4T and P3K5T reveal narrowing of peak widths and significant blue-shifting, respectively. The resulting Gaussian-like peaks are expected, as they represent disruption of pi-pi stacking interactions associated with the addition of surfactant. In contrast, P3K7T displayed no hysochromic shifts, but instead 
gained a more sharply defined absorbance peak and vibronic structure at 560 and $600 \mathrm{~nm}$, respectively. Additionally, P3K6T initially demonstrated a broad and featureless low wavelength $\lambda_{\max }$ at $430 \mathrm{~nm}$. Over the course of 90 minutes, gradual red-shifting results in a new $\lambda_{\max }$ from $430 \mathrm{~nm}$ to $550 \mathrm{~nm}$, accompanied by a strong vibronic structure at $590 \mathrm{~nm}$. This time-dependent chromism is consistent with previously reported literature of the 1:1 molar P3K6T:STS ${ }_{16}$ complex. As such, quaternary ammonium surfactants of different alkyl-chain lengths $\left(\mathrm{STS}_{10,12,14)}\right.$ were complexed with P3K6T in 1:1 molar ratios, and their spectra were compared to that of the 1:1 P3K6T:STS ${ }_{16}$ supramolecular complex (Figure 24a).
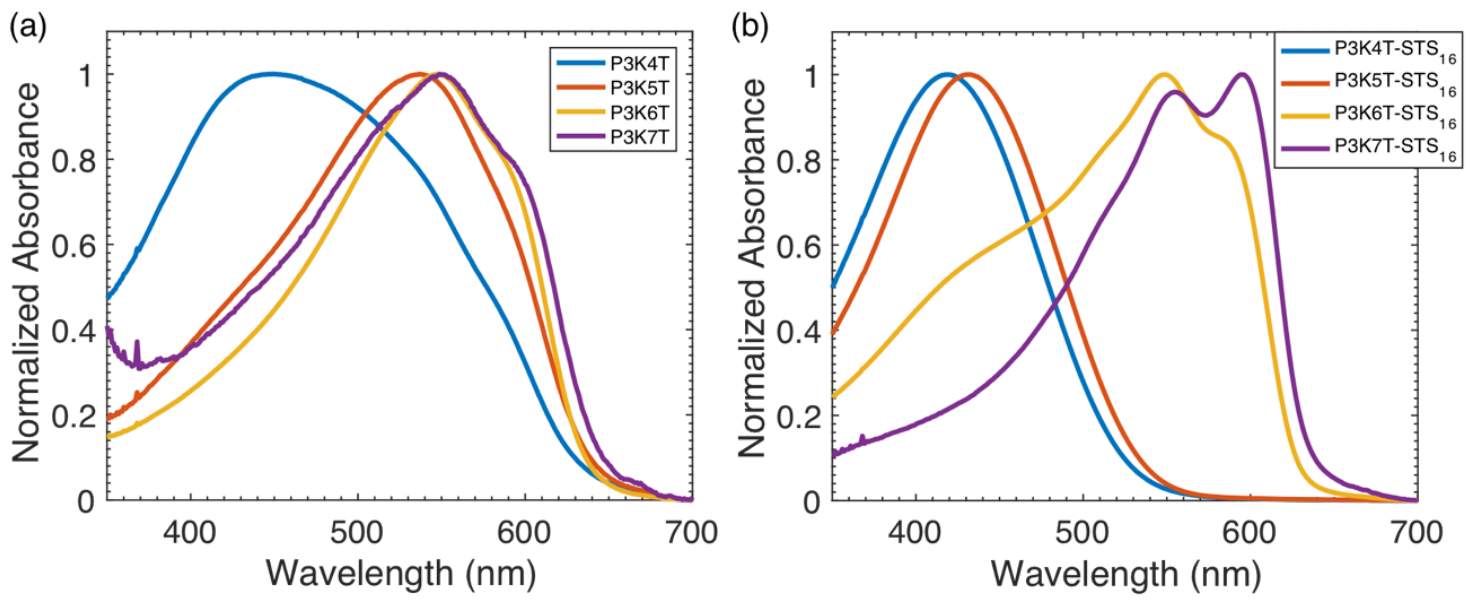

Figure 23. UV-vis spectra of dilute solution P3KnTs of varying alkyl-chain length $\left(\mathrm{n}=4,5,6\right.$, and 7) (a) before and (b) after complexation with STS ${ }_{16}$ over $90 \mathrm{~min}$. The concentration of all solutions is $0.25 \mathrm{mM}$.

The ensuing trend from the addition of varying alkyl-chain length surfactants is similar to that observed in Figure 23b, with increasing and decreasing alkyl-chain lengths promoting bathochromic and hypsochromic shifts, respectively. The red- 
shifted peaks present in Figures 23b and 24a are characteristic of high aspect ratio arrangements of the polythiophene backbone. The apparent vibronic structures present in red-shifted peaks are indicative of rod-like conformations, while their low absorptivity relative to the corresponding $\lambda_{\max }$ is attributed to minimal inter-chain aggregation ensued from the effects of $\pi-\pi$ stacking ${ }^{80}$. It has been acknowledged that regioregular P3AT's adopt their ordered rod-like structures through anticoplanar arrangements ${ }^{81}$, increasing effective conjugation length of the polythiophene backbone and minimizing charge trapping ${ }^{82}$. Conversely, rotational defects between adjacent repeat units are attributed to disordered coil-like states, inducing broad hypsochromic peaks in the UV-visible spectra ${ }^{81}$. These defects lead to heightened torsional angles between thiophene rings, resulting in perturbations of the effective backbone conjugation $^{81}$. By decreasing the conjugation length, the band-gap energy between the HOMO and LUMO molecular orbitals is increased and the wavelength expressed in the absorbance spectrum is reduced. Figure $24 \mathrm{a}$ and $23 \mathrm{~b}$ demonstrate that by increasing the chain length of polythiophene or surfactant, while holding the other constant, upholds the formation of rod-like configurations. As such, the spectroscopic data suggests the incorporation of long alkyl-chains inhibits free rotation of the P3KnT backbone, promoting planarized rod-like conformations and minimal inter-chain aggregation. Therefore, the results presented thus far demonstrate the importance that surfactant alkyl-chain length and architecture can have on the arrangements of aqueous polythiophene-based CPEs. To further demonstrate this effect, the outcome of increased grafting density was studied by incorporating double-tailed 
quaternary ammonium surfactant $\left(\mathrm{DTS}_{16}\right)$ onto the backbone of 1:1 P3K6T:STS ${ }_{16}$ supramolecular complexes.
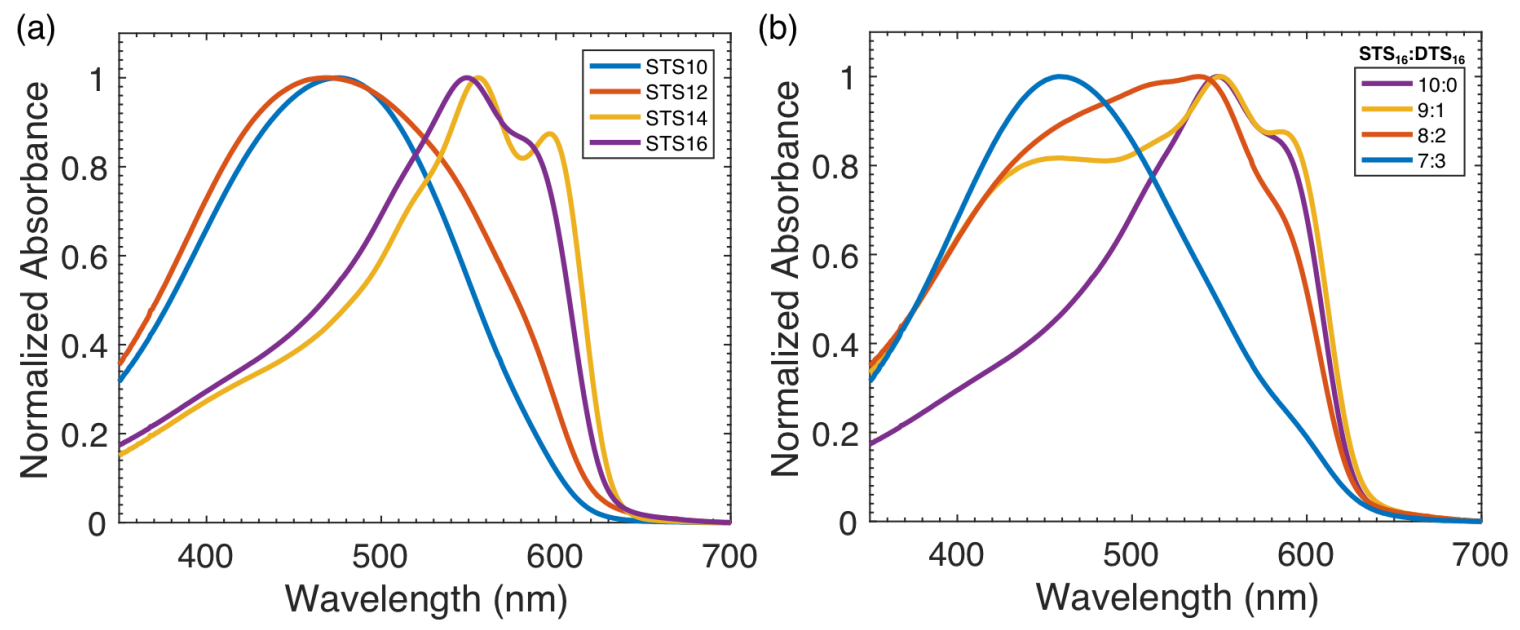

Figure 24. UV-vis spectra of (a) P3K6T complexed in 1:1 molar ratios with various single tailed surfactants $\mathrm{STS}_{\mathrm{n}-1}$ and (b) with various ratios of STS ${ }_{16}$ : DTS ${ }_{16}$. The concentration of all solutions is $0.25 \mathrm{mM}$ and the aging time is $90 \mathrm{~min}$.

The spectrum resulting from additions of $\mathrm{STS}_{16}$ and $\mathrm{DTS}_{16}$ surfactants in overall 1:1 molar ratios $(9: 1,8: 2$, and $7: 3)$ onto the P3K6T backbone can be seen in Figure 24b. Gradual blue-shifts of absorption maxima in the UV-Vis spectrum result from the increased loading of DTS ${ }_{16}$. This may be attributed to the incorporation of double hydrocarbon tails, which produces high grafting density and enhanced bulky sterics between polythiophene and surfactant alkyl-chains. We believe the enhanced hydrophobicity of DTS ${ }_{16}$ alkyl-chains promotes coiledlike structures to minimize interfacial area with the surrounding medium. As these steric effects are not distributed uniformly throughout the polythiophene backbone (not in 1:1 molar ratios) kinks and bends can result between the thiophene rings, 
leading to reductions in torsional angles and promotions of coiled-like states. At low loading of DTS ${ }_{16}$ these kinks are minimal, and therefore strong red-shifts and a time-dependent chromism are observed in the P3K6T-9:1 STS $16:$ DTS $_{16}$ supramolecular complex. Conversely, increased loading of DTS ${ }_{16}$ results in a greater number of kinks, inhibiting the coil-to-rod transition and exhibiting hypsochromic shifts in the UV-vis spectrum. Importantly, no time-dependent chromisms were observed in the $8: 2$ or 7:3 complexes.

In principle, it can be assumed that the addition of $\mathrm{DTS}_{16}$ onto the P3K6T backbone in 1:1 molar ratios would promote planarized chain segments along the polymer backbone. In this instance, uniform sterics between DTS ${ }_{16}$ alkyl-chains would over whelm conformational chain collapse due to hydrophobicity. However, backbone loading of DTS 16 above $30 \%$ lacks sufficient solubility in aqueous media to complete the complexation reaction. As such, the previously discussed hypothesis cannot be addressed through direct experimentation. Instead, the importance of uniform sterics along the P3K6T backbone was investigated using single-tailed surfactant (STS). Previously, Figure 23a demonstrated that separate 1:1 molar additions of $\mathrm{STS}_{10}$ and $\mathrm{STS}_{14}$ along the P3K6T backbone would result in coil-like and rod-like conformations, respectively. Furthermore, the formation of a charge-neutral supramolecular complex of P3K6T and STS 16 led to rod-like planarization of the polymer backbone. As such, it would be expected that a 1:1 P3K6T-surfactant supramolecular complex consisting of varying ratios of STS $_{16}:$ STS $_{10}$ would have similar spectroscopic transitions to that of the P3K6TSTS $_{16}:$ DTS $_{16}$ samples of Figure 24b. Indeed, the results presented in Figure 25a 
after a 40 min equilibration time display just that. Gradual blue-shifting is apparent with increased loading of STS 10 , which is known to promote coil-like states of P3K6T. Such gradual transitions are obvious in the spectra of Figure 24b, but less so in that of $24 a$, which displays spectroscopic transitions resembling a stepchange. Moreover, the spectra of Figure 25b exhibit little to no blue shifting with the increase of STS 14 along the polythiophene backbone, consistent with previous observations of the 1:1 P3K6T:STS ${ }_{14}$ spectrum. Therefore, the coupling of data between Figures 24 and 25 collectively suggest gradual spectroscopic transitions result from a lack of uniform sterics along the polythiophene backbone.
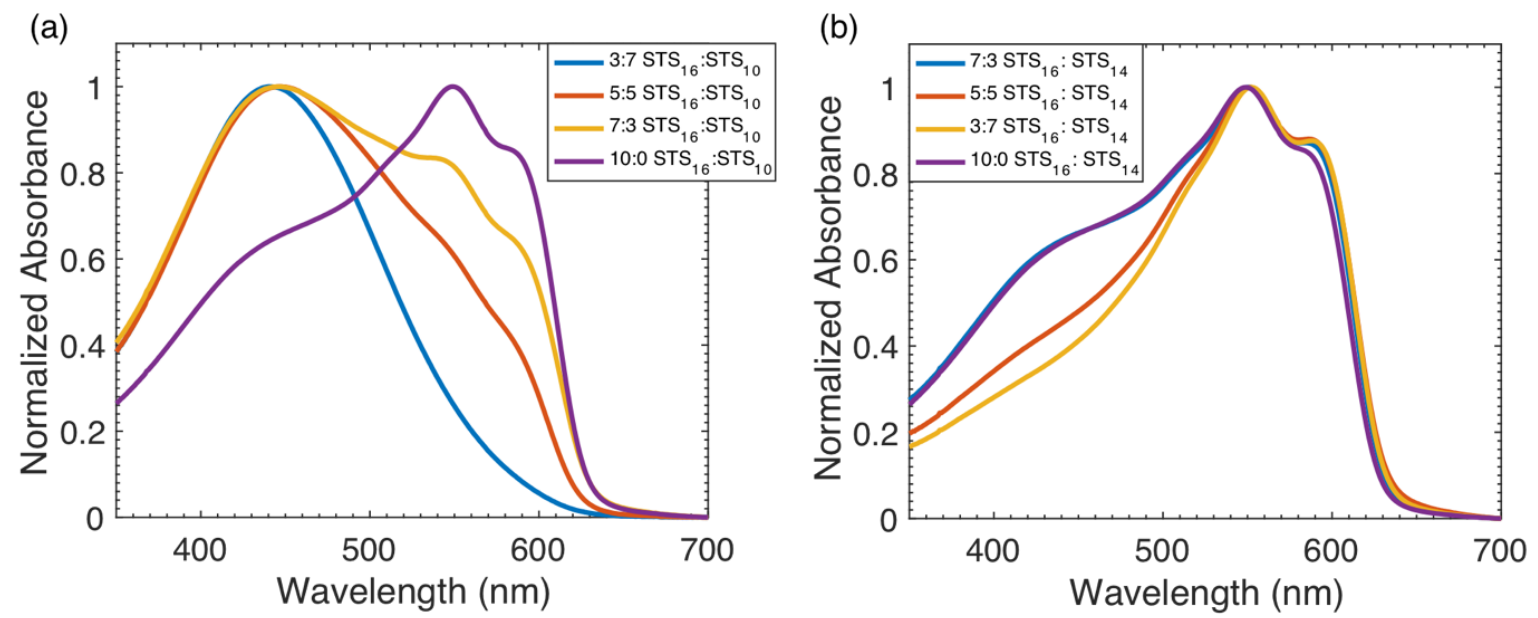

Figure 25. UV-vis spectra of (a) P3K6T complexed in 1:1 molar ratios with (a) various ratios of $\mathrm{STS}_{16}: \mathrm{STS}_{10}$ and (b) various ratios of $\mathrm{STS}_{16}: \mathrm{STS}_{14}$. The concentration of all solutions is $0.25 \mathrm{mM}$ and the aging time is $40 \mathrm{~min}$. 


\subsubsection{Time-Dependent Chromism}

Upon dilution of 1:1 P3K6T:STS 16 and P3K6T-9:1 STS 16 :DTS 16 hydrogel complexes, a distinct color change from orange to dark purple occurs. Furthermore, supramolecular complexes that display red-shifting would precipitate over week long time scales (Figure 26). No chromism was observed in stock hydrogels. These behaviors are consistent with previous observations of the dilute 1:1 P3K6T:STS ${ }_{16}$ system $^{71}$.

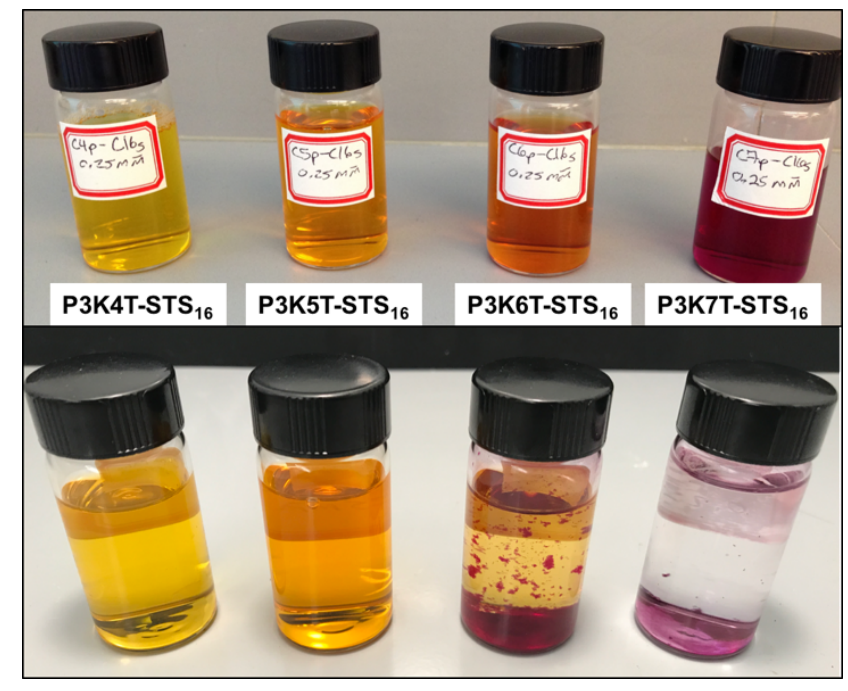

Figure 26. Images of aqueous 1:1 P3KnT:STS ${ }_{16}$ supramolecular complexes at (top) 90 minutes and (bottom) two weeks after dissolution.

UV-visible spectrophotometry was used to measure time-dependent optical alterations of the dilute 1:1 P3K6T:STS ${ }_{16}$ and P3K6T-9:1 STS 16 :DTS 16 complexes. Figures $27 \mathrm{a}$ and $27 \mathrm{c}$ show the time-dependent spectroscopic properties of newly dilute solutions. Over the course of ninety minutes, UV-Vis spectra show gradual shifting's of the initial $430 \mathrm{~nm}$ absorbance maximum to longer wavelengths transpiring at room temperature. A pronounced isosbestic point is shown in both 
spectra, which is indicative of two existing phases in solution ${ }^{33}$; In this instance, both a disordered coil and ordered rod-like state, consisting of 430 and $550 \mathrm{~nm}$ absorbance maxima, respectively. These characteristics are almost identical between 1:1 P3K6T:STS ${ }_{16}$ and P3K6T-9:1 STS ${ }_{16}:$ DTS $_{16}$ solutions.

(a)

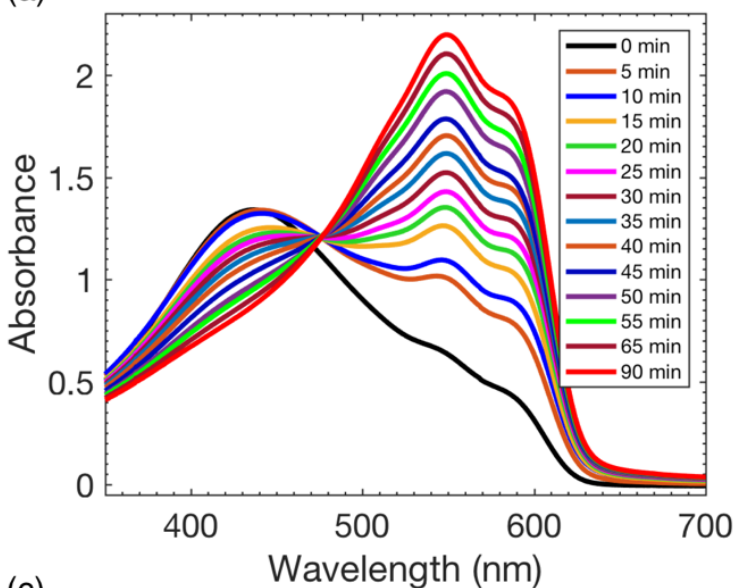

(c)

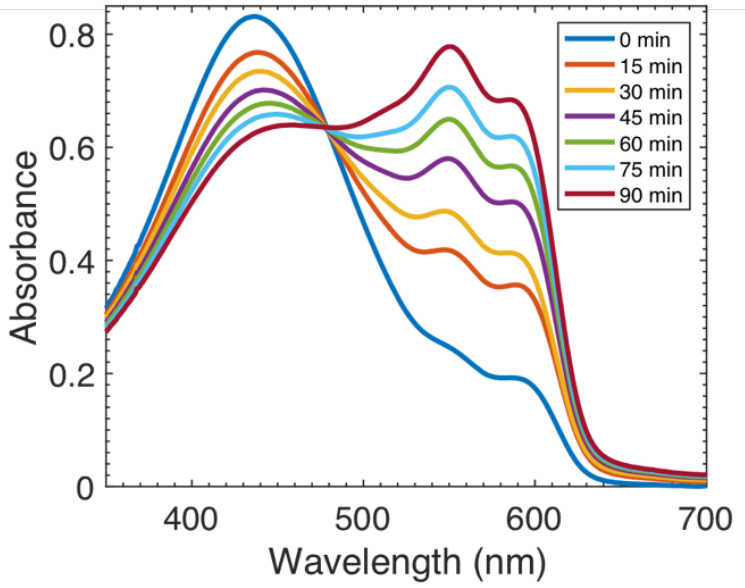

(b)

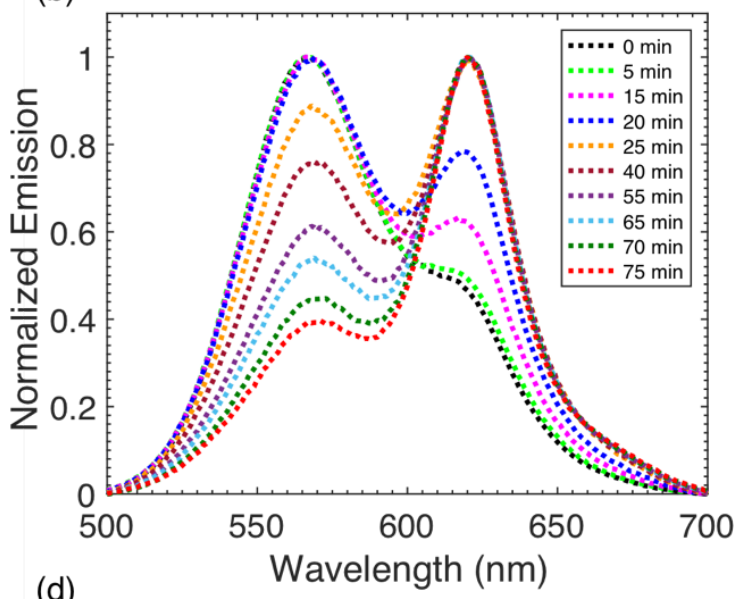

(d)

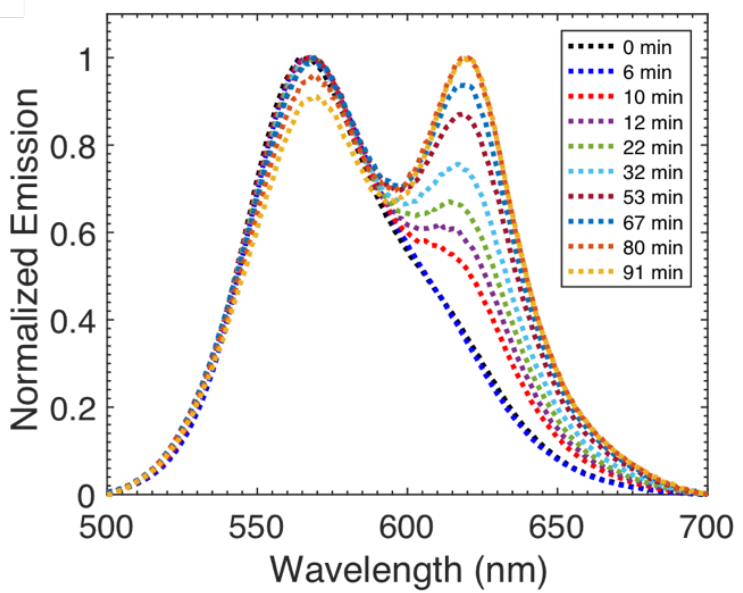

Figure 27. Time-dependent chromism of dilute solutions of the supramolecular complexes at $25^{\circ} \mathrm{C}$. (a) UV-vis and (b) fluorescence spectra of 1:1 P3K6T:STS ${ }_{16}$; (c) UV-vis and (d) fluorescence spectra of P3K6T-9:1 STS 16 : DTS ${ }_{16}$. The concentration of all solutions is $0.25 \mathrm{mM}$. 
In order to provide additional support for the above-mentioned conversion, emission spectra of the 1:1 P3K6T:STS 16 and P3K6T-9:1 STS 16 :DTS 16 supramolecular complexes were generated using fluorescence spectroscopy. These spectra are presented in Figures $27 \mathrm{~b}$ and $27 \mathrm{~d}$. No substantial photoquenching is observed and the emission maximum of both supramolecular complexes gradually shifts to higher wavelengths over time. This is consistent with the observed bathochromic shifts of the corresponding absorbance spectra. The Stokes shift, which is defined as the difference between the emission and excitation maximas $\left(\lambda_{\mathrm{ex}}=430 \mathrm{~nm}\right)$, provides information about vibrational relaxations of excited state fluorophores. In this case, the Stokes shift of the supramolecular complexes is minimized from $130 \mathrm{~nm}$ (coil-like state) to $50 \mathrm{~nm}$ (rod-like state), supporting minimized charge trapping due to polymer backbone extensions $^{71}$. The P3K6T-9:1 STS 16 :DTS 16 supramolecular complex shows minimal shifting with regard to the $430 \mathrm{~nm}$ emission peak compared to that of the 1:1 P3K6T:STS ${ }_{16}$ supramolecular complex. This suggests that the P3K6T-9:1 STS $_{16}:$ DTS $_{16}$ supramolecular complex favors coil-like states compared to that of the 1:1 P3K6T:STS ${ }_{16}$ supramolecular complex. This theory is supported by the consequent absorbance spectra, which shows greater absorbance of the $590 \mathrm{~nm}$ (rod) peak with time relative to that of the $430 \mathrm{~nm}$ (coil) peak for the 1:1 P3K6T:STS ${ }_{16}$ supramolecular complex; the absorbance for the $590 \mathrm{~nm}$ (rod) peak is comparatively reduced for P3K6T-9:1 STS $16:$ DTS $_{16}$ supramolecular complexes. 


\subsubsection{Transition Kinetics}

Further insight into the nature of the coil-to-rod transition was investigated by way of kinetics measurements. UV-Vis absorption spectroscopy was utilized to determine the time-dependent chromism rate with regards to the 1:1 P3K6T:STS ${ }_{16}$ and P3K6T-9:1 STS 16 :DTS 16 supramolecular complexes. As shown in Figure 28, the method of initial rates was employed to determine the rate law of P3K6Tsurfactant supramolecular complexes ${ }^{83}$. Intriguingly, the kinetics data exhibits an inverse first-order rate law for both samples, demonstrating the rate of transition from a coil to rod will increase with decreasing concentration. In theory, $\mathrm{R} \propto \mathrm{C}^{\mathrm{n}}$ where $\mathrm{R}$ is the rate, $\mathrm{C}$ is the concentration, and $\mathrm{n}$ is an exponent denoting the reaction order. In this case, the 1:1 P3K6T:STS ${ }_{16}$ and P3K6T-9:1 STS $16:$ DTS $_{16}$ supramolecular complexes were found to scale with the concentration as $\mathrm{R} \propto$ $\mathrm{C}^{-1.05 \pm 0.12}$ and $\mathrm{C}^{-1.04 \pm 0.05}$, respectively. Such results indicate the mechanism of the coil-to-rod transition is uni-molecular and of intramolecular origin. As concentrations of the dilute solutions deviates from that of the stock hydrogels, the polymer chains become unbound from their viscous gel matrixes and can more easily obtain their extended rod-like conformations. These observations are consistent with the alkyl-chain effects noted above, as an intramolecular driving force is suggestive of side-chain interactions that would foster the backbone transition from a coil-like to rod-like arrangement. Additionally, the lack of photo quenching in the emission spectra of Figure 27 supports the intramolecular hypothesis. Therefore, a two step self-organization mechanism seems likely regarding the coil-to-rod transition of P3K6T-surfactant supramolecular 
complexes. After the coil-to-rod transition takes place, rod-like aggregates form through $\pi-\pi$ stacking. Therefore, the second step of the intramolecular mechanism explains the observed precipitates noted previously in Figure 26. The promotion of such intramolecular rearrangements may be explained through the incorporation of sufficiently long surfactant alkyl-chains, which sterically prevent an intermolecular single-step aggregation mechanism from occurring. As such, the incorporation of surfactant increases the intrinsic weighing factor of the coil-to-rod term from equation 2. 
(a)

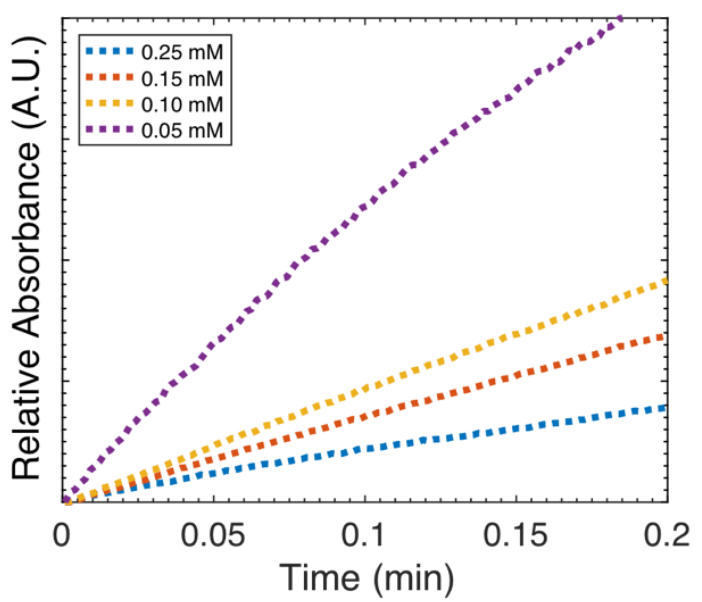

(c)

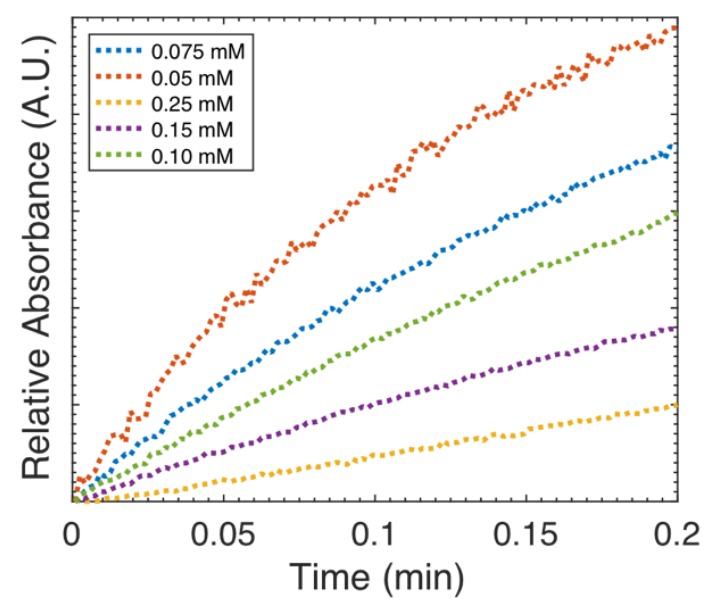

(b)

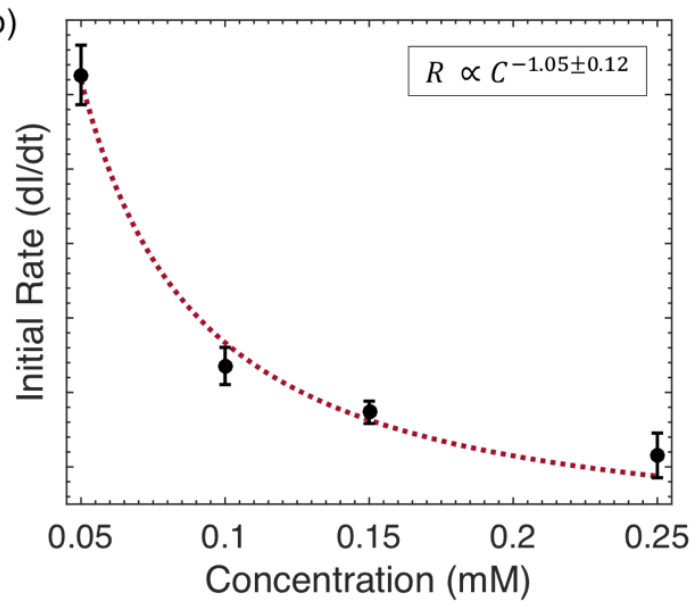

(d)

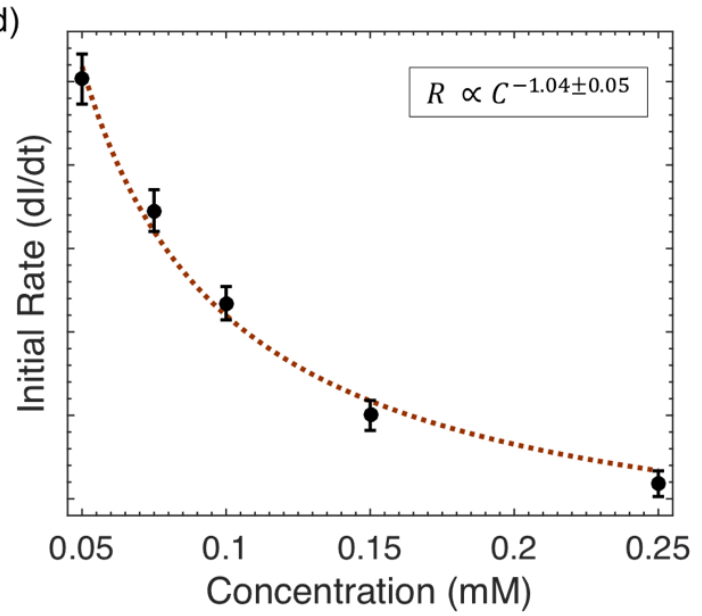

Figure 28. Kinetics of the coil-to-rod transitions of diluted solutions of supramolecular complexes at $25^{\circ} \mathrm{C}$. (a) Time-dependent absorbance at $\lambda=590$ $\mathrm{nm}$ for 1:1 P3K6T:STS 16 , (b) the dependence of initial rate on polymer concentration for 1:1 P3K6T:STS ${ }_{16}$, (c) time-dependent absorbance at $\lambda=590 \mathrm{~nm}$ for P3K6T-9:1 STS $16:$ DTS $_{16}$, and (d) the dependence of initial rate on polymer concentration for P3K6T-9:1 STS $16:$ DTS $_{16}$.

In an attempt to increase the intrinsic weighting factor of rod aggregation in equation 2, solutions of 1:1 P3K6T:STS ${ }_{16}$ were constructed at higher concentrations, and their assembly kinetics was investigated through the same 
methods described above. Interestingly, more noise is introduced into the timedependent absorbance spectra of Figure 28a as the sample concentration approaches $0.60 \mathrm{mM}$. Above this concentration, linear fits of the growth curves initial rates would yield $\mathrm{R}^{2}$ values below 0.50 , and were not considered reliable. Therefore, growth curves of the 1:1 P3K6T:STS 16 supramolecular complex were investigated between concentrations of $0.25 \mathrm{mM}$ and $0.60 \mathrm{mM}$. Fitting of the data presented in Figure 28b yields a rate law with $R \propto C^{-1.07 \pm 0.09}$. The inverse first order rate law is consistent with the kinetics addressed above in Figure 27. As such, no alteration of the intrinsic weighing term from equation 2 was achieved. However, such an alteration may require greater concentrations to promote rod aggregations between substantially long surfactant alkyl-chains.

(a)

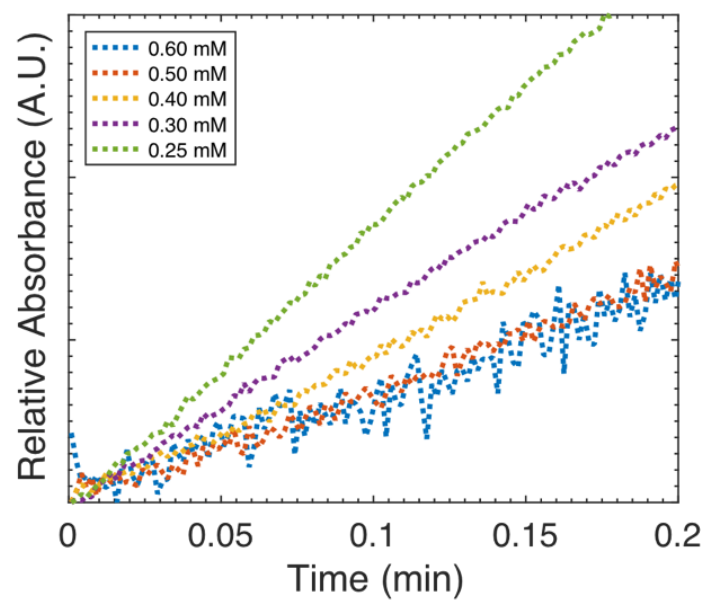

(b)

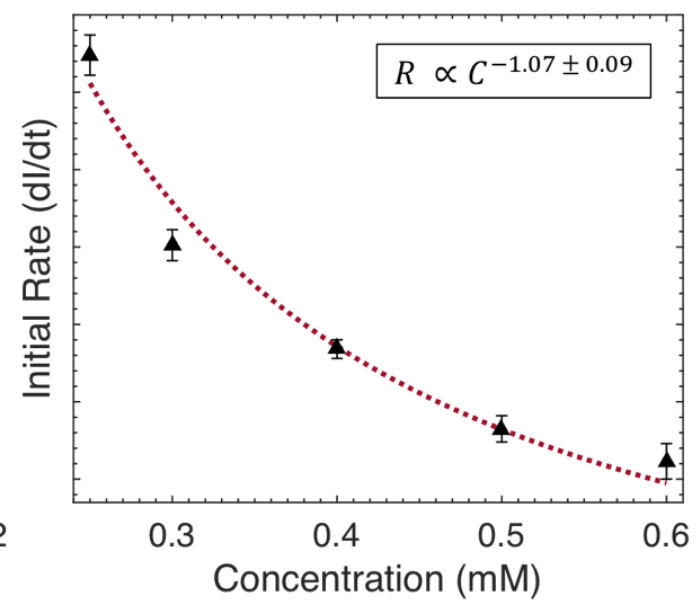

Figure 29. Concentrated kinetics of the coil-to-rod transition of supramolecular complexes at $25^{\circ} \mathrm{C}$. (a) Time-dependent absorbance at $\lambda=590 \mathrm{~nm}$ against aging time of $1: 1$ P3K6T:STS 16 , (b) the dependence of initial rate on polymer concentration for 1:1 P3K6T:STS 16 . 


\subsubsection{Correlation Between ECL and Intrinsic Rigidity}

It has been demonstrated that the persistence length $\left(L_{p}\right)$, a measure of intrinsic chain rigidity within a polymer backbone, may be calculated from the crossover region $\left(q^{*}\right)$ between the -1 and -2 slope of the Porod plot, where $q^{*}\left(l_{p}\right)=$ $\frac{\pi}{6}{ }^{84}$. This indicates longer rod-like segments in the chain will move $\mathrm{q}^{*}$ to smaller values and more coil-like chains move $\mathrm{q}^{*}$ to larger values. The SAXS data of the aqueous solutions at $0.6 \% \mathrm{w} / \mathrm{w}$ was collected for varying ratios of STS 16 : DTS 16 in the overall 1:1 P3K6T:surfactant supramolecular complexes. The data presented in Figure 30 displays increasing q-values accompanied with declining scattering intensity, which approximates the function, $\mathrm{I}(\mathrm{q})=\mathrm{q}^{-1}$ for $\mathrm{q}>0.041 / \AA$ and $\mathrm{I}(\mathrm{q})=\mathrm{q}^{-2}$ for $q<0.021 / \AA$, with the point of transition between functions varying regarding the ratio of $\mathrm{STS}_{16}$ and DTS 16 . Fitting both regimes with a power law enables the determination of these transition points. Within the literature, it is agreed upon that the fractal dimensions of scattering objects relate to the slopes of the log-log Porod plot $^{85}$. On smaller size scales (high q), solutions of conjugated polymer chains have been reported as rod-like in nature, while their coil-like or sheet-like twodimensional structure is observed at larger size scales $(\text { low } q)^{86,87}$. Consistent with the above-mentioned data, spectroscopy of dilute solution polythiophene conformations have been described as consisting of simultaneous coil-like and rod-like structures, affecting the effective conjugation length ${ }^{88,71}$. Previous spectroscopic studies in dilute aqueous solutions have shown the P3K6Tsurfactant complex's effective conjugation length to decline with a pronounced blue shift at higher temperatures ${ }^{71}$. Taking this into consideration, the effective 
conjugation and persistence lengths are expected to be directly proportional, a consequence of the dihedral angle between thiophene units being zero for a truly rigid chain segment.

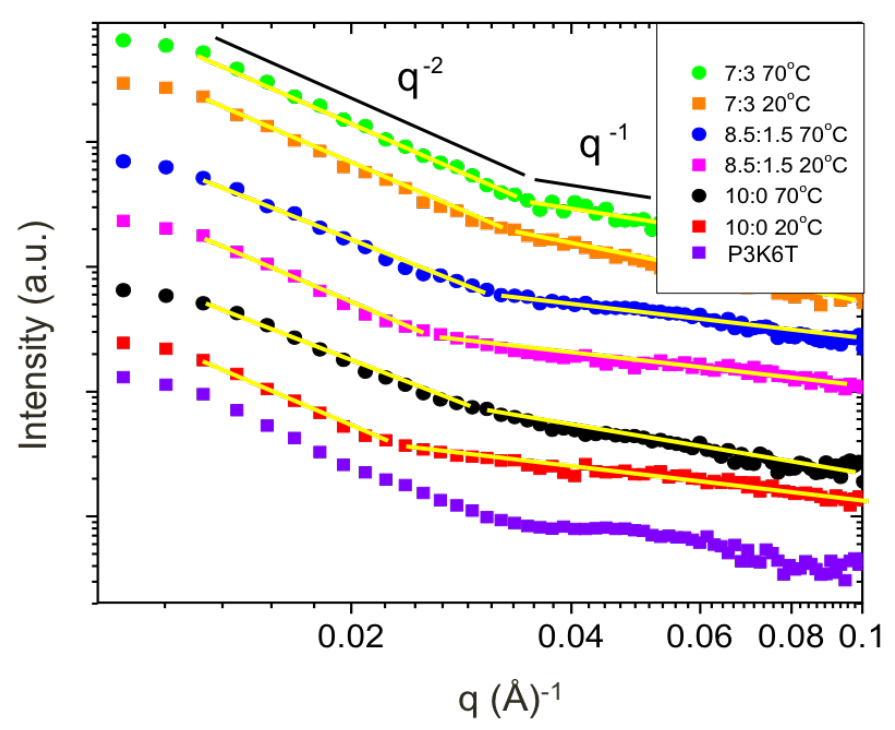

Figure 30. Room temperature SAXS profiles of aqueous solutions of P3K6T supramolecular complexes ( $\sim 0.60 \mathrm{wt} \%)$ with different STS ${ }_{16}:$ DTS ${ }_{16}$ ratios and pure P3K6T ( 0.20 wt \%).

As can be seen from Table 1, higher temperatures and more DTS ${ }_{16}$ content lead to the persistence length of the complex being reduced. These changes in persistence length agree with the spectroscopic data recorded for each complex, and their explanation in terms of the hydrophobic effect. The trend in thermochromistic properties given in Table 1 is also consistent with previous literature, where P3ATs adopt more coiled-like states at higher temperatures due to an entropic driving force that minimizes the Gibbs free energy ${ }^{71}$. For the P3K6T- 
7:3 STS $_{16}:$ DTS $_{16}$ supramolecular complex, the slope crossover is hardly noticeable ( $\mathrm{q}^{*}$ is not well-defined) suggesting that the complex has a coil-like conformation at all measured length scales ${ }^{86}$. Accordingly, increasing the temperature shows little change to the scattering pattern for the P3K6T-7:3 STS ${ }_{16}:$ DTS $_{16}$ supramolecular complex. The spectroscopic data outlined in Figure 24b supports this proposal, as $30 \%$ DTS $_{16}$ loading along the P3K6T backbone shows little to no red shift, indicating the polymer chain is in its fully coiled conformation.

The persistence lengths shown in Table 1 provide estimations for the length of rigidly segmented scattering entities. The length of an entirely rigid chain (contour length) may be calculated using equation 6

$$
l_{c}=\frac{M_{w}}{M_{o}} M_{l}
$$

where $I_{c}$ is the contour length, $M_{w}$ is the weight average molecular weight, $M_{o}$ is the molecular weight of a single monomer, and $M_{1}$ is the length of a thiophene unit. Assuming a monomer length of $0.446 \mathrm{~nm}$, monomer weight of $246 \mathrm{~g} / \mathrm{mol}$, and a supplier provided weighted average of $60 \mathrm{~kg} / \mathrm{mol}$, the contour length of a single P3K6T chain is $103 \mathrm{~nm}$. Furthermore, it is expected that the contour length of the chain be much larger compared to that of the persistence length, otherwise the polymer would be a solely rigid body. According to the calculated persistence lengths listed in Table 1, the P3K6T complexed supramolecules have rigid regions stretching the length of approximately 18 monomer units $(\sim 8 \mathrm{~nm})$, depending upon 
the surfactant architecture. This value is slightly larger than those reported for $\mathrm{P} 3 \mathrm{HT}$, which are usually well below $5 \mathrm{~nm}$, depending upon the solvent ${ }^{88}$.

Table 1. Calculated Persistence Lengths for P3K6T- STS 16 :DTS ${ }_{16}$ Supramolecular Complexes.

\begin{tabular}{cccc}
\hline $\mathbf{S T S}_{16}:$ DTS $_{16}$ & Temperature $\left(\mathbf{C}^{\circ}\right)$ & $\mathbf{q}^{*}\left(\AA^{-1}\right)$ & Persistence Length $(\AA)$ \\
\hline $10: 0$ & 20 & 0.0243 & 78.6 \\
$8.5: 1.5$ & 20 & 0.0251 & 76.1 \\
$7: 3$ & 20 & 0.035 & 54.6 \\
$10: 0$ & 70 & 0.0312 & 61.2 \\
$8.5: 1.5$ & 70 & 0.0325 & 58.8 \\
$7: 3$ & 70 & 0.035 & 54.6 \\
\hline
\end{tabular}

\subsubsection{Lyotropic Liquid Crystalline Self-Assembly}

A consequence of P3K6T backbone extensions, due to heightened persistence lengths, is the increase of aspect ratios along the backbone's rigid segments in solution. From a theoretical standpoint, such rigid chain segments have been shown to result in the formation of LC polymer arrangements ${ }^{89}$. Moreover, the relation between polymer persistence lengths and the onset of lyotropic ordering may be codified through phase-boundary diagrams. The comparative onset of LC textures between differing polythiophene-surfactant supramolecular complexes can be used to infer if aspect ratios are maintained from dilute to concentrated solutions. In this case, the critical concentration $\left(\mathrm{c}^{*}\right)$ required to induce LLC phase transitions is taken as the onset of optical 
birefringence. Polarized optical microscopy (POM) may therefore be employed to study this behavior in rigid polymers.

Induced birefringent properties are the result of two limiting cases with the preferment of molecular orientation relative to a director (or vector) ${ }^{90}$. The first is homogeneous orientation, which calls for the alignment of macromolecules perpendicular to the film normal. The second, homeotropic alignment, defines the LC director orientation as parallel to the film normal. In many cases, uniform orientation of LC films can be achieved through an induced mechanical shear, which may produce a periodic banded structure that is known to show alterations in the molecular director of lyotropic LC polymers ${ }^{90}$, including polythiophenesurfactant supramolecules ${ }^{59}$. Figure 31 shows POM images of 1:1 P3K6T- STS 16 liquid crystalline textures, both before (Figure 31a) and after (Figure 31b) applied mechanical shearing without removal of the coverslip. A smokey LLC texture is presented in Figure 31a, which is characteristically reported for the hexagonal LLC phase of surfactant molecules ${ }^{91}$. Additionally, the presence of a banded texture in Figure $31 \mathrm{~b}$ suggests the polymer backbone is perpendicular to the bands, and thus the application of a mechanical shear force can act to align the polymer backbone along the shear direction ${ }^{59}$. 
(a)

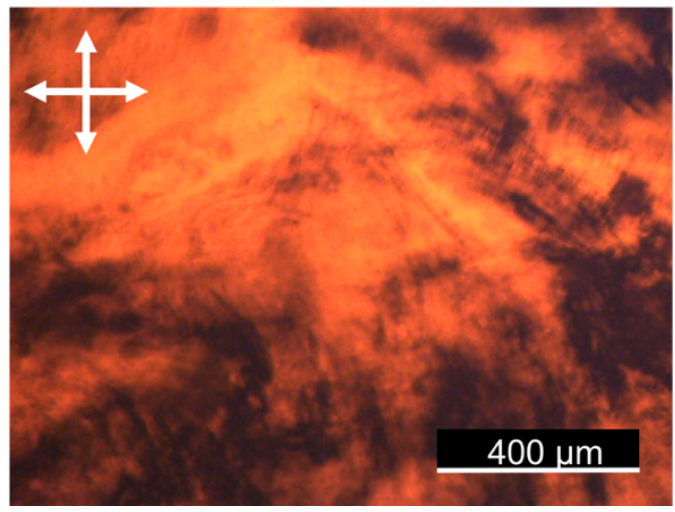

(b)

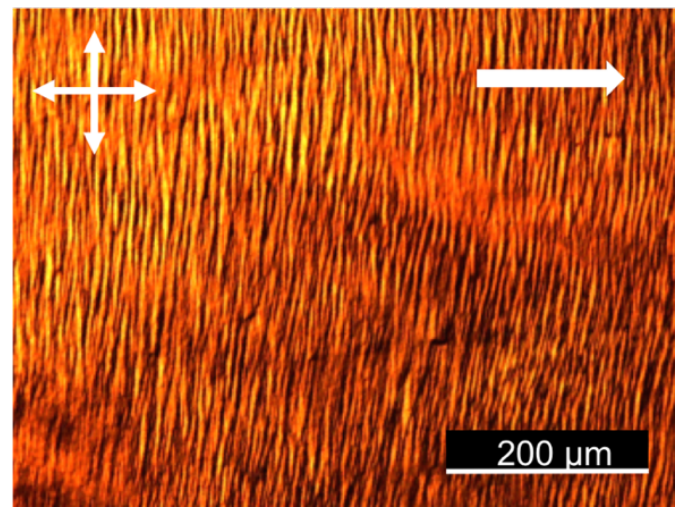

Figure 31. Optical images of $51 \mathrm{wt} \%$ aqueous solutions of the $1: 1$ P3K6T-STS 16 supramolecular complex under crossed polarizers. (a) Without mechanical shearing and (b) with shearing. The arrow in part b indicates the shear direction.

By way of POM, the phase diagrams constructed in Figure 32 display the relationships between surfactant chain length/architecture and $c^{*}$ for both 1:1 P3K6T:STS 16 and P3K6T-9:1 STS $16:$ DTS $_{16}$ supramolecular complexes. Error bars denote the onset of heterogeneous and homogenous birefringence in the samples. The alterations of $c^{*}$ displayed in Figure 32 suggests that surfactant chain length and architecture play a substantial role in influencing the LLC behavior of P3K6Tsurfactant supramolecules. Figure 32a demonstrates the effect of surfactant chain length on the concentration required to enter the liquid crystalline phase. Appropriately, $\mathrm{C}^{*}$ is shown to decrease linearly with increasing $\mathrm{STS}_{\mathrm{n}-1}$ alkyl-chain lengths, indicating the presence of greater rod-like character promotes the formation of LLC phases, consistent with absorption spectroscopy measurements. Juxtaposed, Figure $32 \mathrm{~b}$ shows the variation of $c^{*}$ as a function of DTS ${ }_{16}$ loading onto the polythiophene backbone in the overall 1:1 P3K6T:surfactant complex. Distinct from Figure 32a, Figure 32b shows an almost exponential relationship, a 
possible result of non-uniform sterics along the polythiophene backbone. These results are consistent with those obtained from the in-situ SAXS measurements presented in Figure 30 and Table 1. The increased loading of DTS ${ }_{16}$ onto the P3K6T backbone obstructs the increase of persistence length, promoting a more coiled-like state and shortening the effective length of the polymer chain. Within experimental error, there is little difference seen between $c^{*}$ at $0 \%$ and $10 \%$ loading of DTS ${ }_{16}$. The similar surfactochromic properties and persistence lengths of both complexes is indicative of their rod-like nature. As such, the similarities in their values of $c^{*}$ is expected and thus justified. Furthermore, $c^{*}$ values for $20 \%$ and $30 \%$ DTS $_{16}$ loading show notable increases, a consequence of their anticipated coil-like nature as determined from trends in UV-vis spectra and in-situ SAXS measurements.

(a)

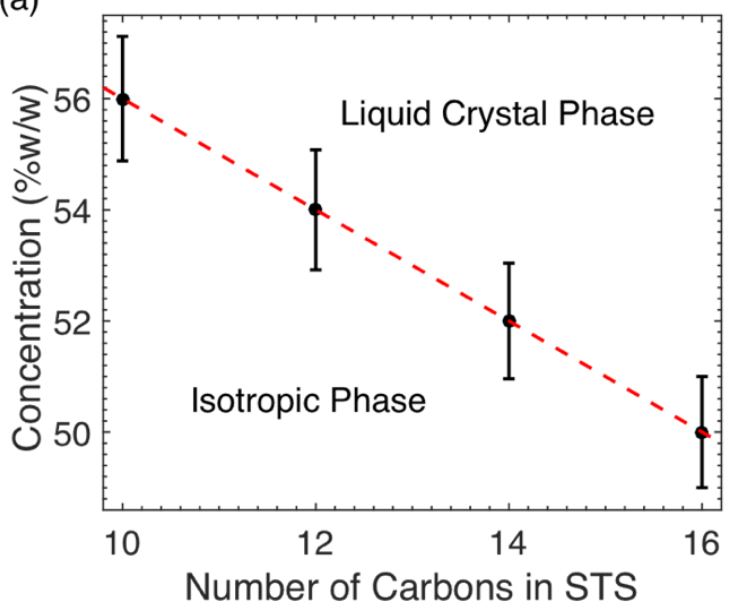

(b)

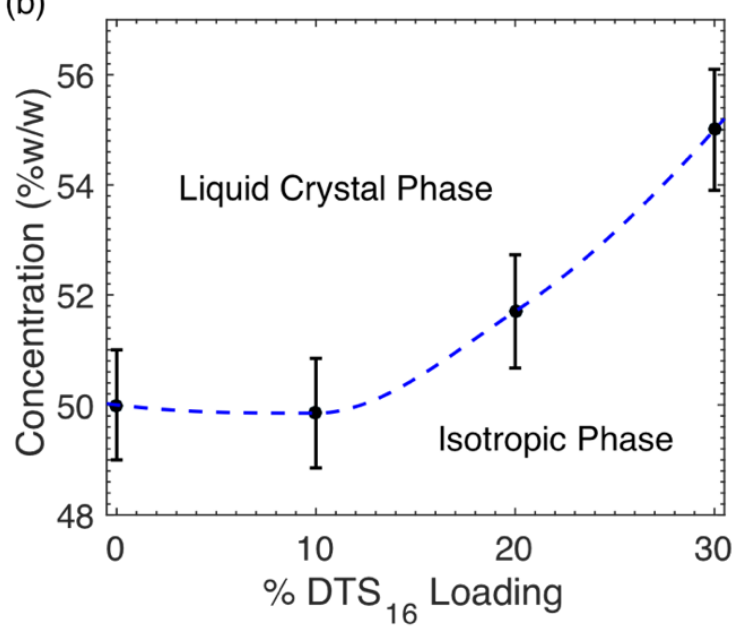

Figure 32. Room-temperature liquid crystalline phase diagrams of P3K6T supramolecular complexes with (a) varying chain lengths of single tailed surfactants $\left(\mathrm{STS}_{\mathrm{n}-1}\right)$ and (b) varying double tailed surfactant $\left(\mathrm{DTS}_{16}\right)$ loading. Dashed lines guide the eyes only. 
Figure 33a shows SAXS data from $51 \mathrm{wt} \%$ LC aqueous solutions of the 1:1 P3K6T:STS ${ }_{16}$ supramolecular complex. The scattering peaks occur at q-ratios consistent with $1, \sqrt{ } 3,2$, and $\sqrt{ } 7$. This interpretation leads to a d-spacing of $26.8 \AA$. The scattering pattern is consistent with the expected hexagonal symmetry from a hexagonal or columnar LC phase ${ }^{59,92}$. However, morphology interpretation from a limited number of peaks is rather equivocal where alternative morphologies such as, e.g., those from cubic aspect 8 cannot be excluded based upon SAXS alone. Figure $33 \mathrm{~b}$ shows the temperature dependent SAXS profile during heating. Clearly, the long-range symmetry is retained through the heating experiment up to $110{ }^{\circ} \mathrm{C}$, indicating the hexagonal LC phase is considerably stable. At $140^{\circ} \mathrm{C}$, the $\sqrt{ } 3 q^{*}$ peak disappears and the first scattering peak shifts to a lower $q$ value of 0.202 $\AA^{-1}$. Moreover, a small portion of the original peak persists at $q=0.218 \AA^{-1}$. While nematic and lamellar LC phases are predominant in conjugated polymer solutions $^{93}$, the hexagonal LC phase suggested in this work may be attributed to the long surfactant side chains and molecular weight polydispersity of $\mathrm{P} 36 \mathrm{~K}^{94}$. In addition, the hexagonal LC phases in conjugated polymer solutions can also be stabilized by geometrical frustrations ${ }^{95}$. Recently, the formation of the hexagonal LC phase has been reported in a sulfonated polythiophene complexed with the surfactant, centyltrimethylammonium bromide $(\mathrm{CTAB})^{59}$. 

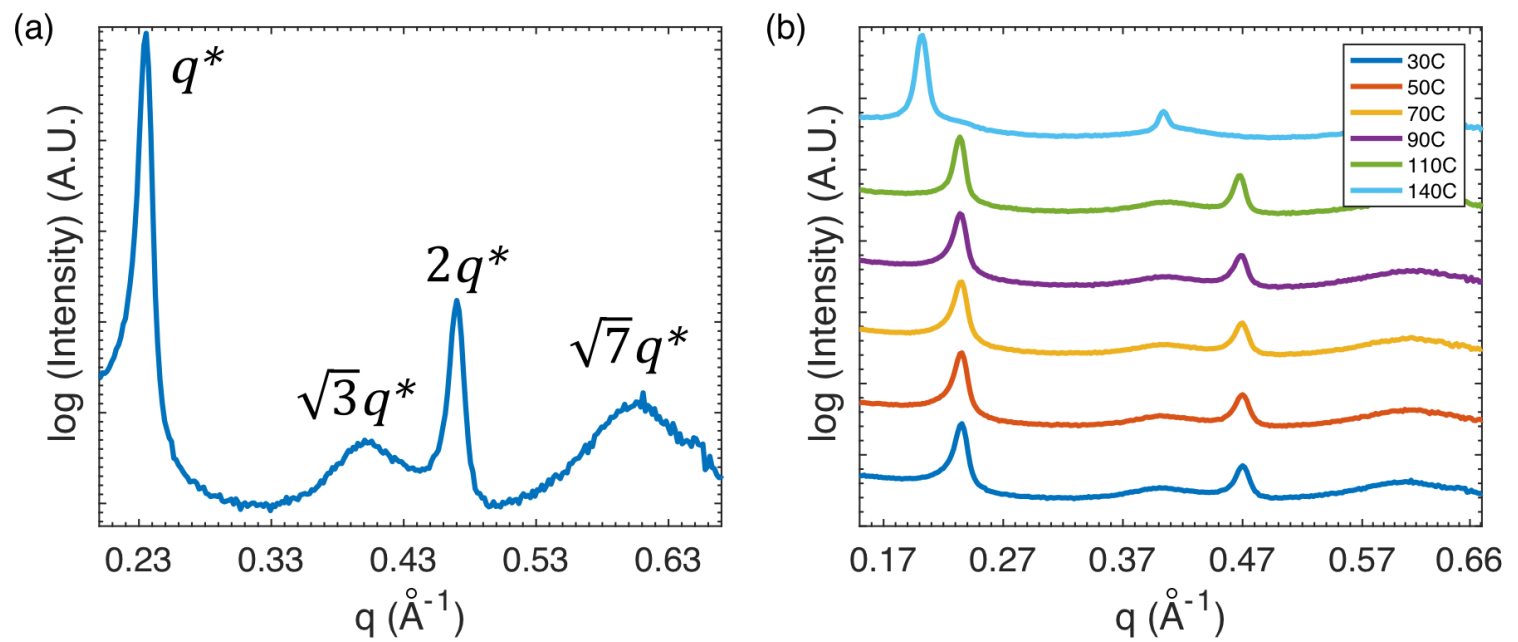

Figure 33. (a) Room temperature SAXS pattern and (b) temperature dependent in situ SAXS profile of the lyotropic solution of the 1:1 P3K6T-STS ${ }_{16}$ supramolecular complex. The concentration of all solutions is $\sim 51 \mathrm{wt} \%$.

\subsubsection{Electrical Properties of P3K6T-Surfactant Thin Films}

Mechanical shearing of 1:1 P3K6T:STS ${ }_{16}$ supramolecular complexes resulting in removal of glass coverslips led to dried films lacking a banded texture, but maintaining the uniform birefringent properties indicative of polymer chain alignment (Figure 34). In these instances, conductivity measurements were made in a four-point probe configuration. Three consecutive linear voltage sweeps were first run with probes perpendicular and then parallel to the shear direction (Figure 35a). The average of these sweeps is shown in Figure 35b with probes both aligned to, and against the direction of shear. When aligned, the produced I-V curves showed a greater slope, an expected result due to theorized molecular uniformity induced through mechanical shearing, implying intramolecular charge transport is being measured. In contrast to this, probes arranged perpendicular to 
the shear direction show a reduced slope, an outcome of the less efficient charge transport through the intermolecular hopping mechanism of $\pi-\pi$ stacking.

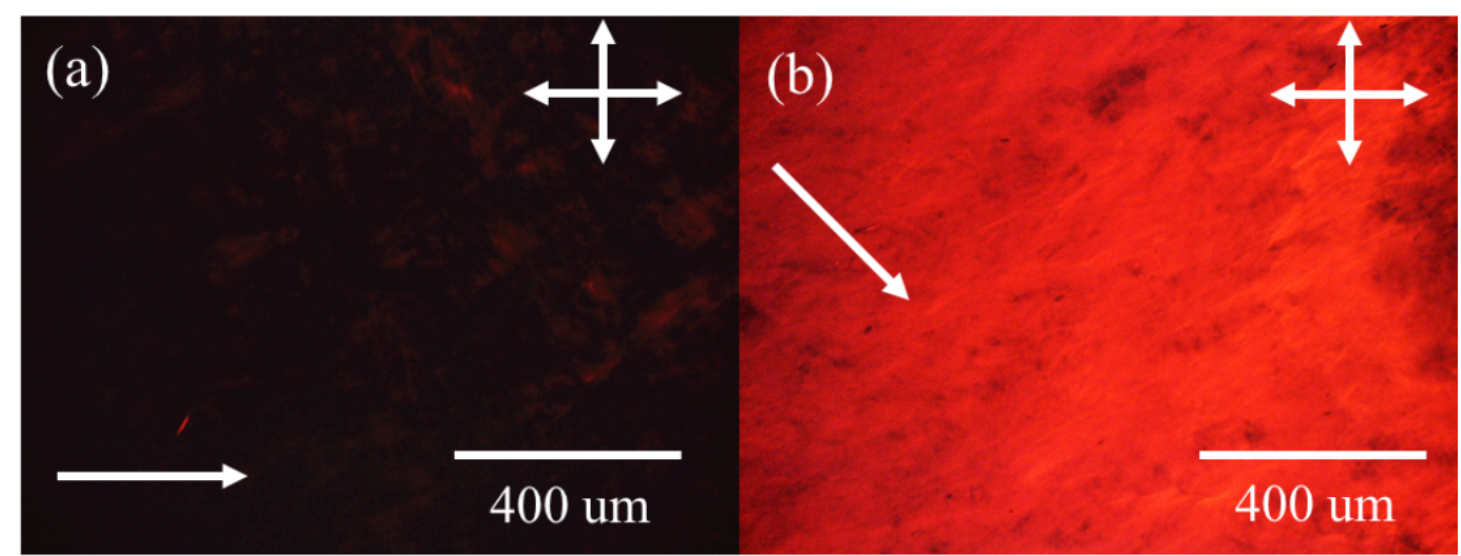

Figure 34. Optical images of a sheared LC film of P3K6T-STS ${ }_{16}$ under crossed polarizers after drying. (a) $0^{\circ}$ rotation and (b) $45^{\circ}$ rotation. The arrows represent the shear direction.

Equation 5 was used to calculate the bulk resistivity of the films, which yielded a ratio of film thickness $(\mathrm{t})$ to probe distance $(\mathrm{s})$ of less than 0.5 . The inverse of the bulk resistivity yields the bulk conductance for the thin film. Therefore, the calculated conductivity of $1: 1$ P3K6T-STS ${ }_{16}$ thin films with probes parallel and perpendicular to the direction of shear were $7.94( \pm 0.73) \times 10^{-4}$ and $2.38( \pm 0.55)$ $x 10^{-4} \mathrm{~S} / \mathrm{m}$, respectively. These values are reasonable when compared to those reported in literature for pure $\mathrm{P} 3 \mathrm{HT}$ in its non-doped state $\left(2.85 \times 10^{-3} \mathrm{~S} / \mathrm{m}\right)^{96}$. 
(a)

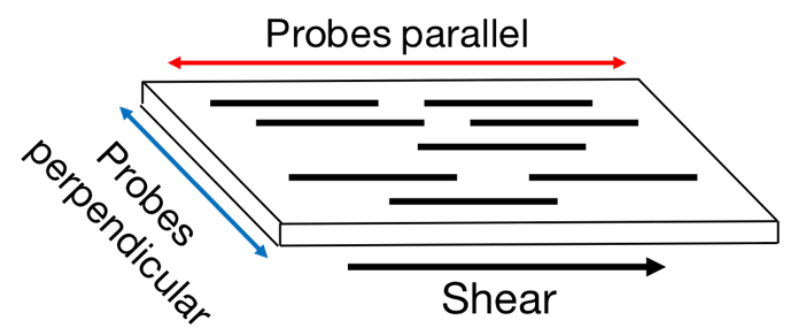

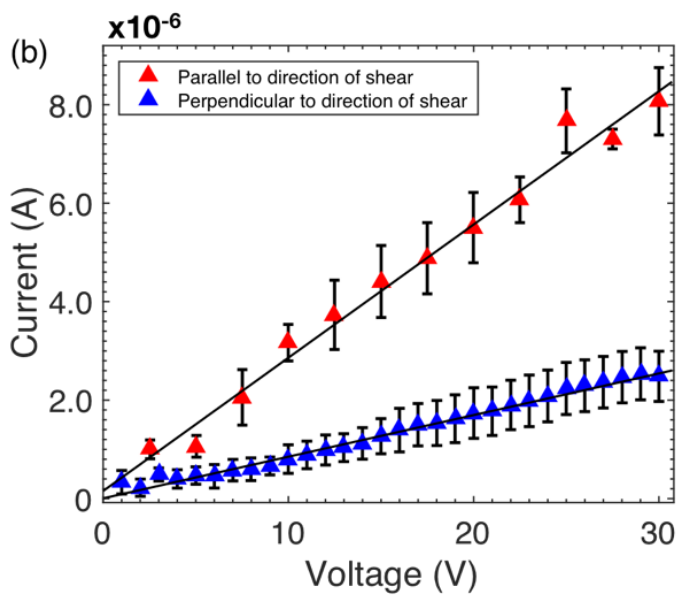

Figure 35. Room-temperature measurements of anisotropic conductivity. (a) Illustration of electrical conductivity measurements and (b) current vs voltage curves of sheared P3K6T-STS 16 LC films after complete drying parallel and perpendicular to the shear direction. 


\subsection{System 2}

\subsubsection{Dilute Solution Properties of P3CnTs}

P3CnTs were prepared in DMSO and diluted to a concentration of 25 $\mu \mathrm{g} / \mathrm{mL}$. The samples were left to equilibrate for 80 minutes. Figure 36 displays the UV-Vis absorption spectra of P3CnTs in pure DMSO. Broad Gaussian-like absorption peaks are observed for both P3C3T and P3C4T with $\lambda_{\max }$ 's of 442 and $464 \mathrm{~nm}$, respectively. In contrast, the absorption of P3C6T exhibits two peaks with narrow half-widths at $590 \mathrm{~nm}$ and $550 \mathrm{~nm}$, corresponding to $\mathrm{I}^{0-0}$ abs and $\mathrm{I}^{0-1}$ abs vibronic transitions, respectively ${ }^{28}$. Interestingly, the spectrum of P3C5T presents the broadest absorption of all P3CnT samples with both $\mathrm{I}^{0-0}{ }_{\text {abs }}$ and $\mathrm{I}^{0-1}$ abs transitions apparent at $590 \mathrm{~nm}$ and $550 \mathrm{~nm}$, respectively, and a primary absorption peak at $500 \mathrm{~nm}$. Therefore, the resulting spectra of P3CnTs collectively display a distribution of bathochromic and hypsochromic shifting as a function of alkyl-chain length. It is well understood that regioregular P3AT's form anti-coplanar arrangements of their thiophene rings, promoting rod-like structures that maximize backbone conjugation and minimize charge traps ${ }^{81}$. Conversely, geometric frustrations between adjacent repeat units are ascribed to coil-like disorder of the polythiophene backbone. This leads to defects in backbone conjugation, which manifests as broad hypsochromic peaks in electronic spectra. This spectroscopic interpretation is similar to that previously discussed for system 1. Figure 36 clearly demonstrates that $\lambda_{\max }$ 'S of P3CnTs gradually red-shift with increasing the length of their side chains, promoting absorption of vibronic fine structures. This effect may be explained through the increase of alkyl-chain sterics between thiophene 
rings, in which longer side-chains force co-planarity of the polymer backbone, encouraging $\pi-\pi$ stacking and increased conjugation length ${ }^{76}$. These findings are consistent with theoretical calculations suggesting ordered assembly and enhanced conjugation lengths may be achieved through the incorporation of long alkyl-chains $^{97}$. Additionally, the absorption spectra of P3CnTs dissolved in DMSO has been reported previously ${ }^{98}$. While the results displayed in Figure 36 show consistency with literature, a notable exception can be observed in the absorption of $\mathrm{P3C} 5 \mathrm{~T}$, which displayed diminished vibronic bands in the cited work. The disparity between the vibronic fine structures of P3C5T may be justified by the 80 minute equilibration time implemented in our study. This suggests a timedependent chromism is present in the absorption spectrum of $\mathrm{P} 3 \mathrm{C} 5 \mathrm{~T}^{71}$.

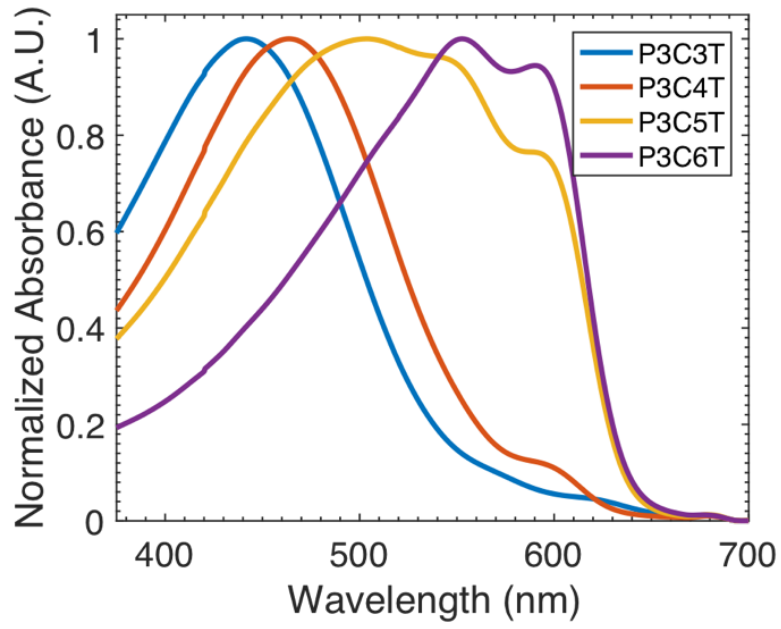

Figure 36. UV-vis spectra of dilute P3CnTs in DMSO with varied alkyl-chain lengths $(n=3,4,5$, and 6$)$ after 80 -minute equilibration. The concentration of all solutions is $25 \mu \mathrm{g} / \mathrm{mL}$. 
Previous reports of numerous P3AT systems has shown their self-organization may be promoted through the addition of a poor or marginal solvent $\mathrm{t}^{29}$. In this way, unfavorable interactions between the polythiophene chains and poor solvent molecules drives self-assembly and aggregation ${ }^{29}$. As such, the degree of assembly should be dependent on the amount of poor solvent present in the system. Figure 37a demonstrates such self-organizations of P3C5T upon initial dissolution $(\mathrm{t}=0 \mathrm{~min})$ in varying ratios of DMSO:EtOH. In pure DMSO (10:0 DMSO:EtOH), P3C5T displays a Gaussian-like absorption peak at $460 \mathrm{~nm}$ and lacks vibronic fine structures, similar to that of P3C3T and P3C4T in Figure 36. Upon dissolution of P3C5T in smaller ratios of DMSO:EtOH, the spectra gradually exhibits more red-shifted absorption peaks accompanied by strongly absorbed vibronic structures at 550 and $590 \mathrm{~nm}$. At lower ratios, the $\mathrm{I}^{0-0}$ abs shifts slightly to higher wavelengths. These observations imply the addition of EtOH enhances $\pi-\pi$ stacking between the thiophene rings, thereby promoting aggregation and extended conjugation lengths. Figure 37b shows the spectra of P3C5T dissolved in various ratios of DMSO:EtOH after storage in the dark for 24 hours. In all cases, little to no pronounced Gaussian-like absorption peak is observed at approximately $460 \mathrm{~nm}$. Additionally, more pronounced absorption is detected for vibronic structures in the 550 to $600 \mathrm{~nm}$ range. This suggests greater assembly is upheld with time and that all solutions of P3C5T in mixed solvents display time-dependent spectroscopic transitions. 
(a)

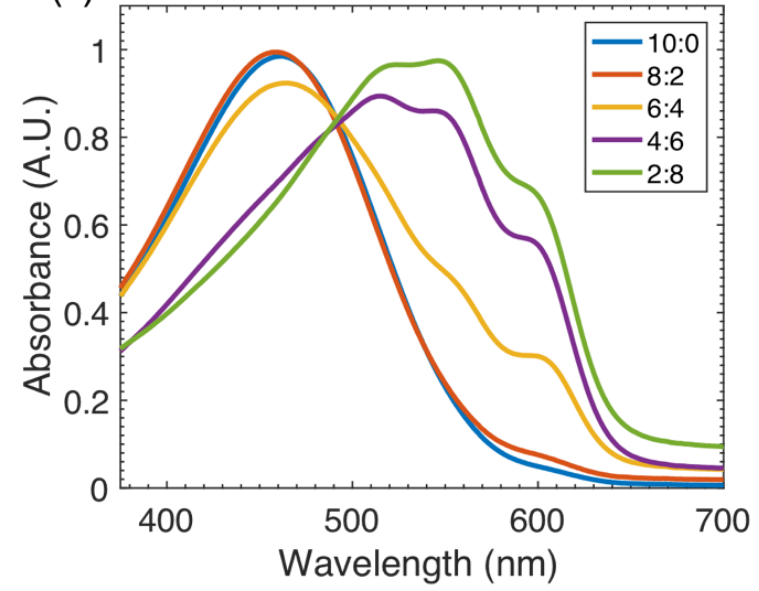

(b)

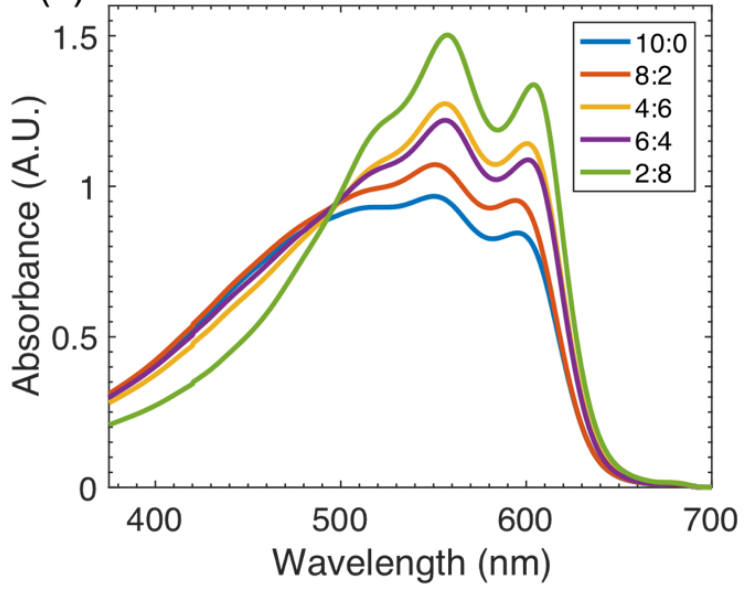

Figure 37. UV-vis spectra (a) upon and (b) 24 hours after initial dissolution of $\mathrm{P} 3 \mathrm{C} 5 \mathrm{~T}$ in varying ratios of DMSO:EtOH. The concentration of all solutions is approximately $25 \mu \mathrm{g} / \mathrm{mL}$.

While the addition of marginal solvent to solutions of P3C5T in DMSO yields an enhancement of backbone co-planarity, the thermochromism of P3AT's have been reported to represent diminished conjugation length ${ }^{99}$. Both solutions of P3C5T in pure DMSO and 8:2 DMSO:EtOH displayed broad Gaussian-like peaks in their UV-vis spectra upon initial dissolution (Figure 37a). As such, the thermochromistic transition of these solutions was investigated over the full transition range and the results are presented in Figure 38. With increasing temperature, both P3C5T in pure DMSO and 8:2 DMSO:EtOH show decreased absorption of vibronic structures at $550 \mathrm{~nm}$ and $590 \mathrm{~nm}$. Additionally, the blueshifting of absorption peaks steadily track with temperature rise, resulting in Gaussian-like peaks at, or higher than $50^{\circ} \mathrm{C}$. These results signify that high temperatures cause polythiophene chains to twist and distort into coil-like 
conformations that maximize the systems overall entropy ${ }^{71,99}$. When temperatures are decreased below a critical value, the polymer chains re-organize to form rodlike structures that minimize the total free energy of the system. Moreover, P3C5T in DMSO lacks any presence of vibronic structures at the highest experimental temperature $\left(80^{\circ} \mathrm{C}\right)$. This is in opposition to $\mathrm{P} 3 \mathrm{C} 5 \mathrm{~T}$ in 8:2 DMSO:EtOH, which shows slight absorptions around 590 to $600 \mathrm{~nm}$ under the same conditions. These results further suggest $\mathrm{EtOH}$ promotes $\mathrm{P} 3 \mathrm{C} 5 \mathrm{~T}$ aggregation and backbone coplanarity.
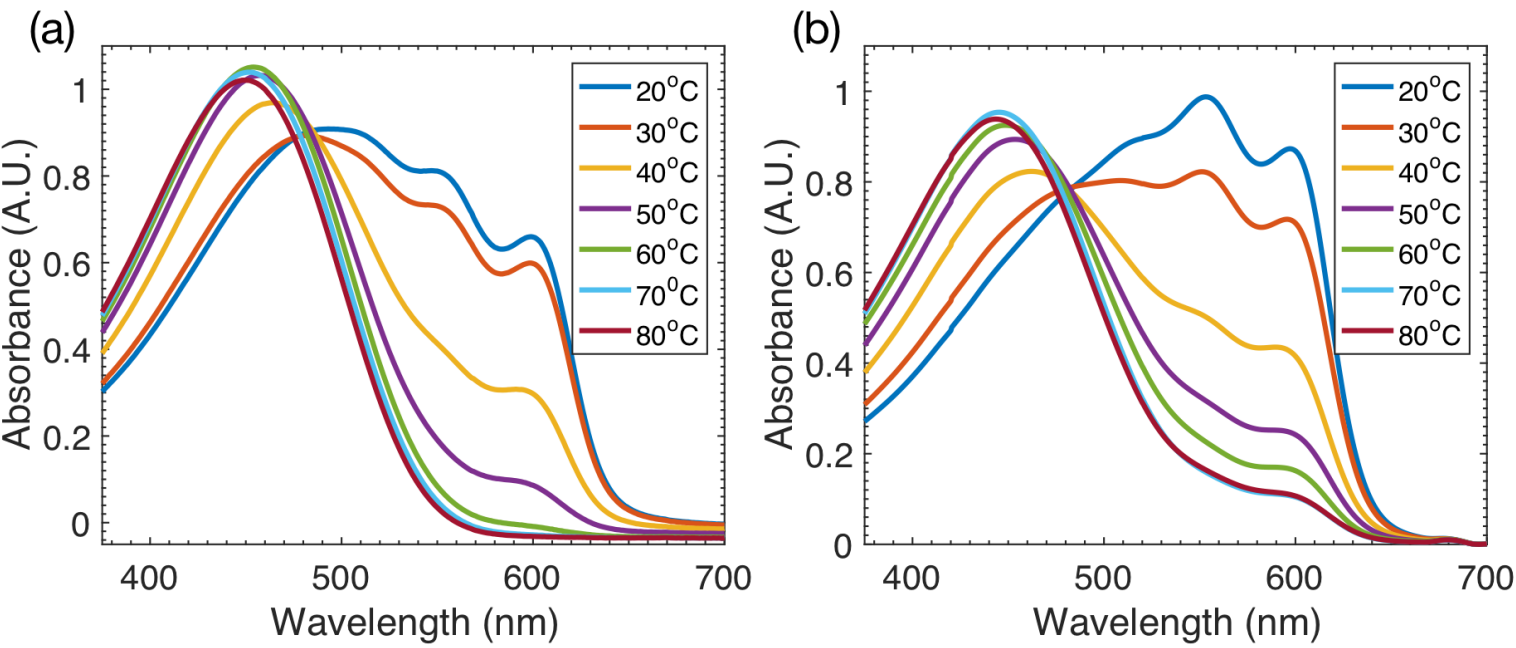

Figure 38. UV-vis spectra of P3C5T thermochromism in (a) DMSO and (b) 8:2 DMSO:EtOH. The concentration of all solutions is approximately $25 \mu \mathrm{g} / \mathrm{mL}$.

\subsubsection{Transition Kinetics of P3C5T}

To attain a deeper understanding of the physical mechanism surrounding P3CnT self-assembly, solutions of P3C5T in DMSO and 8:2 DMSO:EtOH were chosen for time-dependent spectroscopic studies. Figure 39a and 39b show timedependent spectroscopic properties of P3C5T in DMSO and 8:2 DMSO:EtOH, 
respectively. Over time, the solutions gradually red-shift from their initial Gaussianlike 480 to $490 \mathrm{~nm}$ absorption to a broad peak accompanied by vibronic structures at $550 \mathrm{~nm}$ and $590 \mathrm{~nm}$. The existence of a clear isosbestic point is observed in both solutions, confirming the presence of two co-existing phases; in this case, coil-like disordered and rod-like ordered states associated with hypsochromic and bathochromic shifts, respectively ${ }^{71,100}$. Previous reports of P3AT systems indicate that such vibronic peaks at $\lambda=590 \mathrm{~nm}$ are associated with the quantity of rod-like structures in solution ${ }^{31,35}$. As such, the method of initial rates was employed to measure the kinetics of P3C5T solution assembly ${ }^{83}$. In this way, the initial slope of absorbance $(\lambda=590 \mathrm{~nm})$ against aging time is accepted as the initial rate of the spectroscopic transition. Figure 40 shows the kinetics of P3C5T solution assembly in DSMO and 8:2 DMSO:EtOH. In this instance, the initial rate scales with the concentration as $R \propto C^{2.06 \pm 0.12}$ and $R \propto C^{2.05 \pm 0.05}$ for P3C5T in pure DMSO and 8:2 DMSO:EtOH, respectively. These results demonstrate no significant difference in reaction orders between the pure and mixed solvent P3C5T solutions. Therefore, no change in the dynamic mechanism of P3C5T assembly takes place subsequent to the addition of a poor solvent. 
(a)

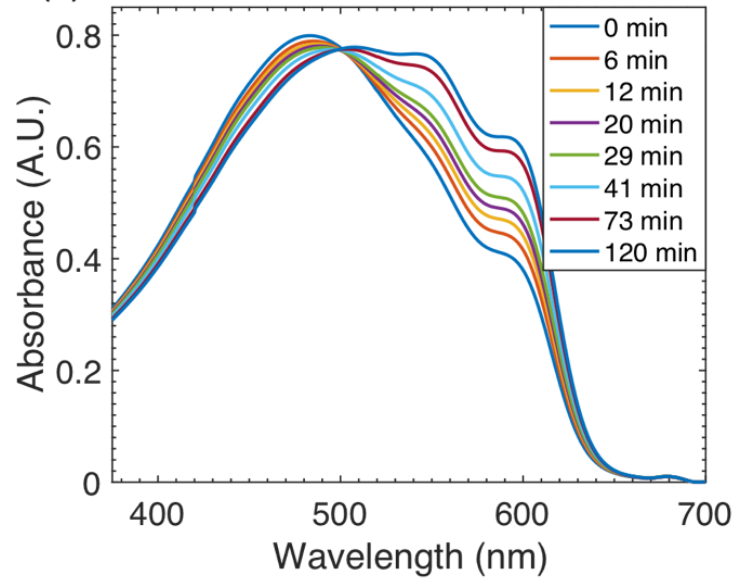

(b)

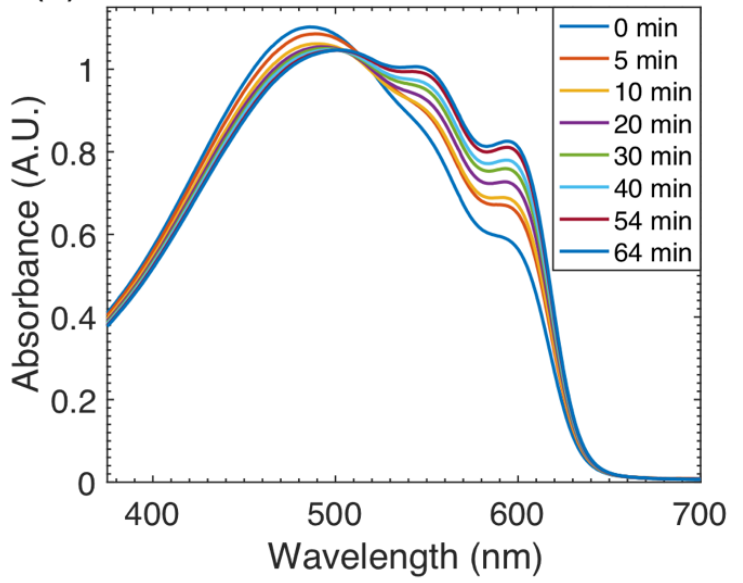

Figure 39. Time-dependent chromism of dilute solutions of P3C5T in (a) DMSO and (b) 8:2 DMSO:EtOH. The concentration of all solutions is approximately 25 $\mu \mathrm{g} / \mathrm{mL}$.

It is suggested that the self-organization mechanism of P3AT assembly results from a coil-to-rod conformational transition and $\pi-\pi$ stacking between adjacent rods to form aggregates of nanofibrils ${ }^{101}$. Again, the polymer nucleation theory may be employed to further understand the dynamics of polythiophene selforganizations. For the coil-to-rod transition, system 1 showed kinetics that demonstrate inverse first order behavior $\left(S_{\text {coil }} \propto C^{-1}\right)$. For rod aggregation, scaling of other systems have established a nucleation rate that is proportional to the concentration raised to the second power $\left(S_{\text {aggreg }} \propto C^{2}\right)^{102}$. In addition, second order spectrokinetic measurements have been reported for other P3AT derivatives with the potential to hydrogen bond ${ }^{103}$. Therefore, the second-order growth kinetics of P3C5T indicates rod-rod aggregation is the main process driving solution assembly. As such, the kinetics suggest the self-organization mechanism of 
P3C5T in DMSO is of a single-step intermolecular origin, as opposed to the intramolecular mechanism of P3K6T-surfactant supramolecular complexes.

The discrepancy between the solution assembly mechanism of system 1 and that of P3C5T may be explained through variations in their chemical structures. The carboxylic acid alkyl-chain end group of P3C5T has the potential to act as both a hydrogen bond donor and acceptor ${ }^{104}$. Accordingly, intermolecular hydrogen bonding is possible between chains of P3C5T, but not between those of 1:1 P3K6T: surfactant supramolecular complexes. Such short range interactions may act to drive aggregations of P3C5T chains, resulting in the aforementioned second order rate law. Therefore, the presence of carboxylic acid functional groups acts to increase the intrinsic weighing factor of the aggregation term in equation 2. 
(a)

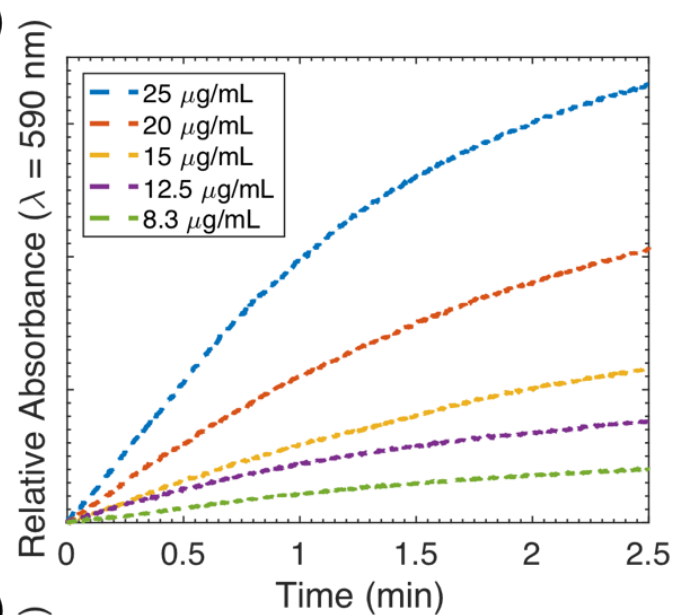

(c)

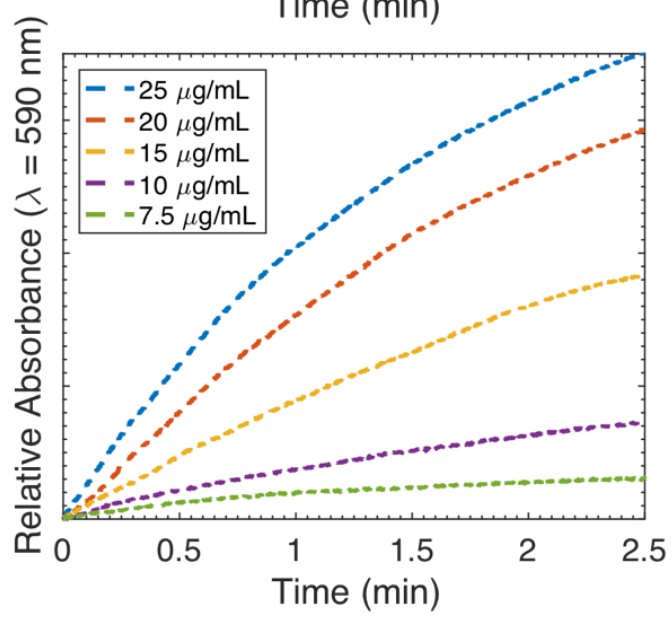

(b)

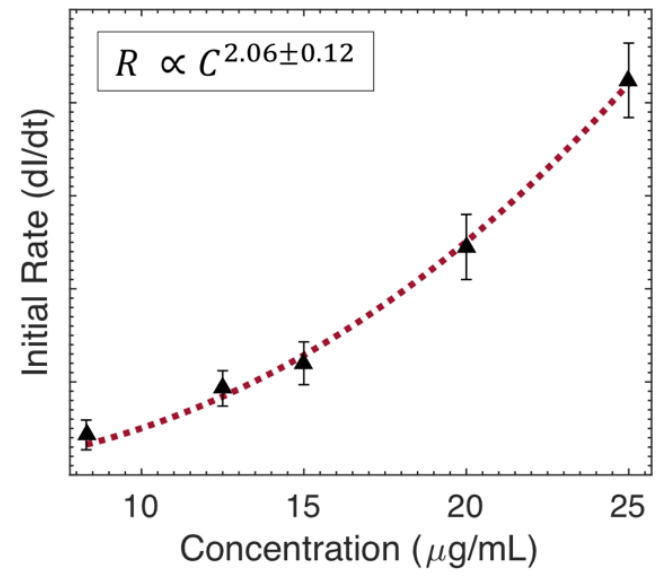

(d)

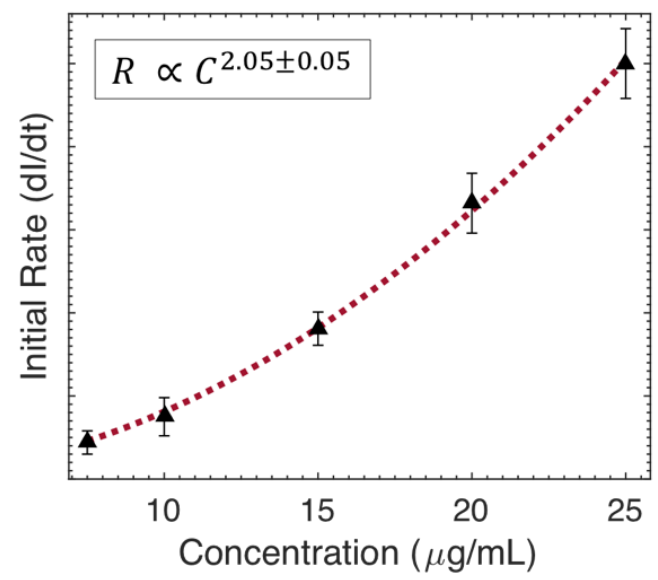

Figure 40. Assembly kinetics of diluted solutions of P3C5T at $25^{\circ} \mathrm{C}$. (a) Timedependent absorbance against aging time of P3C5T in DMSO, (b) the initial rate versus polymer concentration of P3C5T in DMSO, (c) time-dependent absorbance against aging time of P3C5T in 8:2 DMSO:EtOH, and (d) the initial rate versus polymer concentration of P3C5T in 8:2 DMSO:EtOH. 


\subsubsection{Liquid Crystal Gelation of P3C5T}

A consequence of conjugated backbone aggregation influencing the solution assembly of P3C5T is the formation of hydrogen bonded 3-D networks at larger concentrations. Additionally, the use of low-volatility solvents, similar to DMSO, have been found to promote P3AT gelation ${ }^{52}$. The FTIR spectra of P3C5T solid films and gels (30 wt\% in DMSO) were evaluated to determine the extent of hydrogen bonding (Figure 41). In both the solid film and gel, sharp peaks are present within the carbonyl region of the FTIR spectra. In the solid state, the carbonyl vibrations present at $1697 \mathrm{~cm}^{-1}$ are indicative of low energy hydrogen bonding ${ }^{60}$. Reports of P3CnT's have indicated that carbonyl peak positions at approximately $1735 \mathrm{~cm}^{-1}$ are suggestive of free $\mathrm{C}=\mathrm{O}$ stretching ${ }^{60}$. In the gelated state, P3C5T shows carbonyl peaks at roughly $1716 \mathrm{~cm}^{-1}$, which suggests that degrees of hydrogen bonding are still present in the gels. Moreover, re-drying of P3C5T gels shifts carbonyl peaks back to lower energy wavenumbers, signifying complete restoration of solid state hydrogen bonding. This suggest the increase in wavenumber may be attributed to the presence of solvent within the system. 


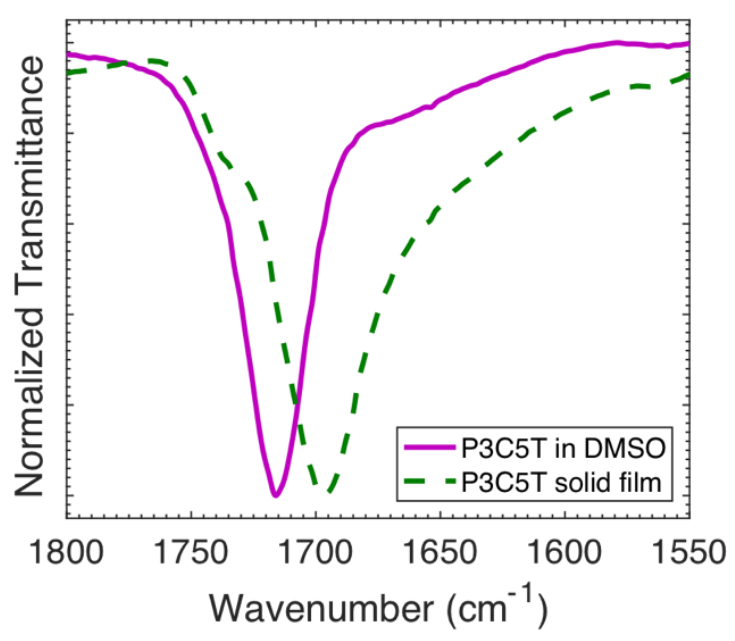

Figure 41. FTIR spectra showing the carbonyl shifting between P3C5T gels and solid films. The concentration of the gel is $30 \mathrm{wt} \%$.

Figure 42 illustrates the bulk and thermal properties of concentrated P3C5TDMSO solutions. In the bulk state, P3C5T solutions maintain fluidity up to concentrations as high as $15 \mathrm{wt} \%$ at room temperature (Figure 42a). Above a critical concentration $(\sim 21 \mathrm{wt} \%)$ the percolation threshold is reached and the solutions gel. Slight color changes may be noticed between less concentrated solutions and that of the P3C5T gel, providing further evidence for the formation of an interconnected 3-D network. With heating, gel films of the P3C5T display a color change from dark purple to bright orange (Figure 42b). Upon cooling back to room temperature, the dark purple color of the films is gradually restored and fluidity is no longer present. These observations imply the gelation process of P3C5T is thermally reversible and supported through a network of hydrogen bonding interactions $^{62}$. Moreover, air drying of P3C5T produces solid films with a metallic sheen that can be carefully removed from glass substrates. 
(a)
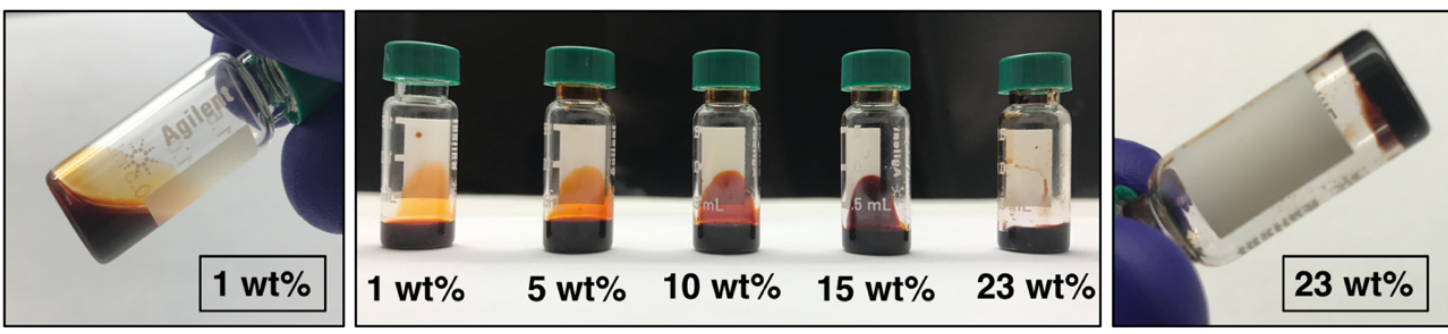

(b)
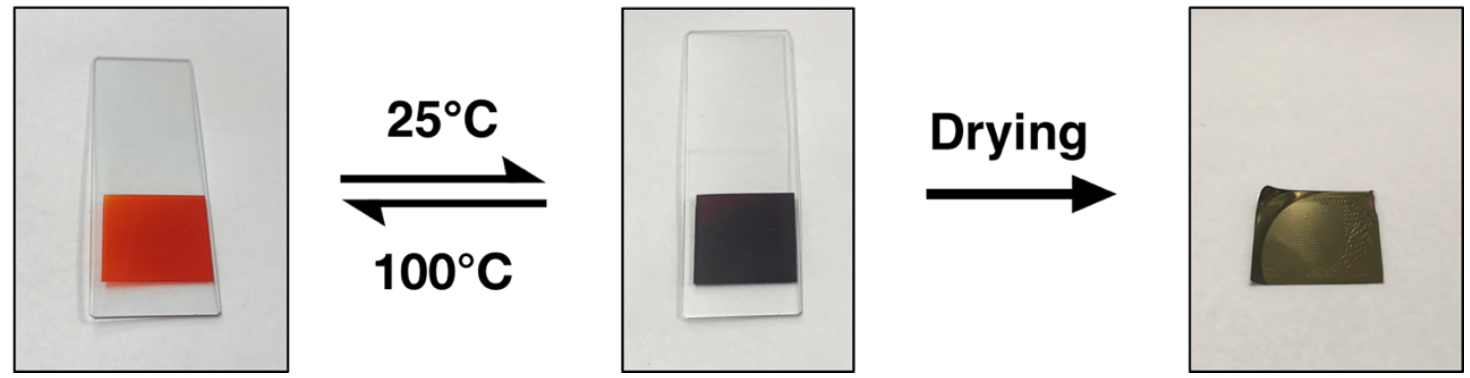

Figure 42. (a) Bulk and (b) thin film characteristics of concentrated P3C5T/DMSO thermoreversible gels. The concentration of all thin films is $23 \mathrm{wt} \%$.

Upon sandwiching the P3C5T gel between a clean glass slide and coverslip with $1 \mathrm{~N}$ of force, the gels exhibit characteristic phenomena related to birefringent media. When the samples are placed under a polarized optical microscope, double diffraction is observed in the form of light and dark contrasting regions. Furthermore, the domains change alternatingly upon rotating the sample through a $45^{\circ}$ angle in-between the microscopes crossed polarizers. The latter and former observations indicate the construction of a liquid crystal, with textures resembling that of a Schlerien nematic phase ${ }^{105}$. Figure 43 illustrates the onset of such LC formation as a function of concentration. The presence of the nematic LC phase suggests that $\mathrm{P} 3 \mathrm{C} 5 \mathrm{~T}$ chains maintain rigid rod-like conformations due to their net anisotropic polarizability ${ }^{16,105,106}$. As such, long-range directional order may be 
supported in P3C5T microstructures through parallel alignment of the polymer long axis $^{59}$, consistent with the finding from system 1 . As the results of $x$-ray scattering measurements did not show any indication of crystalline peaks, further support is provided that the P3C5T gel may form through a network of interconnected rods supported through short range hydrogen bonding ${ }^{106}$. Because POM can identify the onset of optical birefringence indicative of LC ordering, the boundary between the isotropic and nematic LC phase of P3C5T was determined to be $21 \pm 1 \mathrm{wt} \%$ (Figure 43).

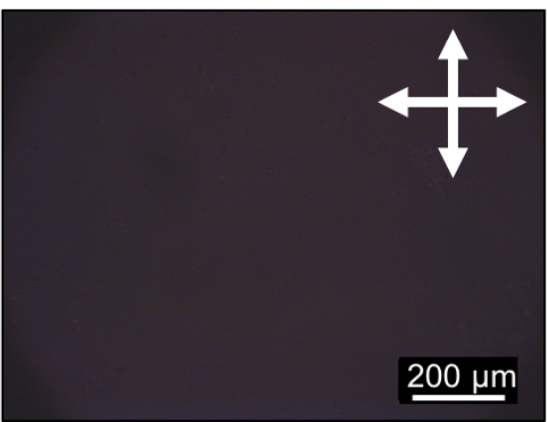

Isotropic

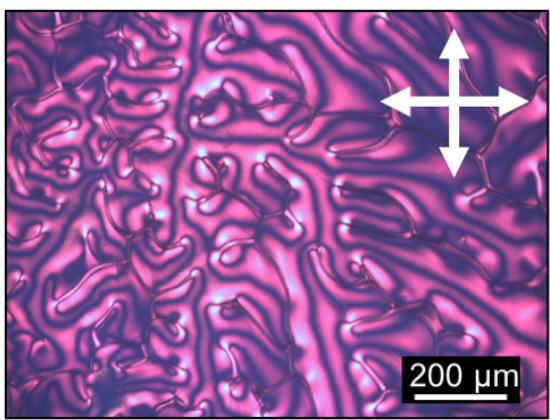

Liquid Crystal

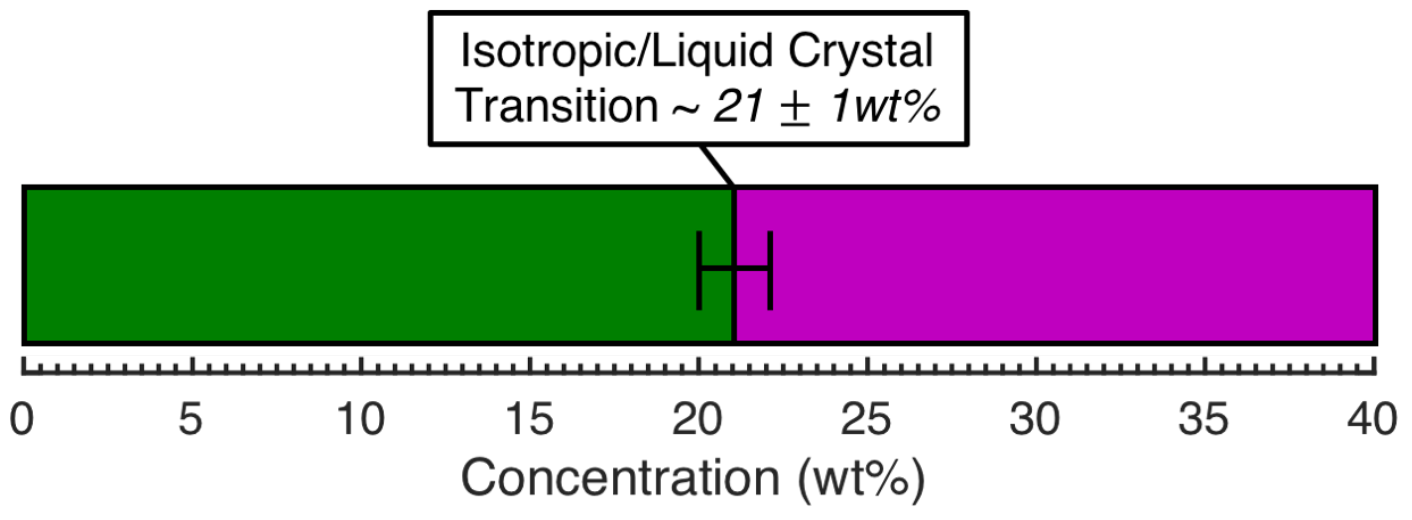

Figure 43. Phase diagram of P3C5T in DMSO LC formation as a function of concentration. Illustrative optical images of isotropic and liquid crystalline phases are displayed above the phase boundary. 
In some instances, P3C5T textures of periodic stripes would appear under glass coverslips. This phenomenon would only occur at low temperature heating over extended periods of time (greater than 18 hours). Figure 44 demonstrates typical images of the periodic banded textures extending radially outward from the coverslip's center. Such phenomena have been observed with solutions of P3HT in trichlorobenzene after addition to glass capillaries ${ }^{107}$. For P3C5T, the period of a single band is approximately $64 \mu \mathrm{m}$ (Figure 44b), similar to the periodicity observed in P3HT. We believe these textures result from a shearing capillary force induced from the sandwiching of P3C5T between two glass substrates. As these textures are not observed consistently, multiple factors including temperature, humidity, and surface effects may play a key role in their materialization ${ }^{107}$.

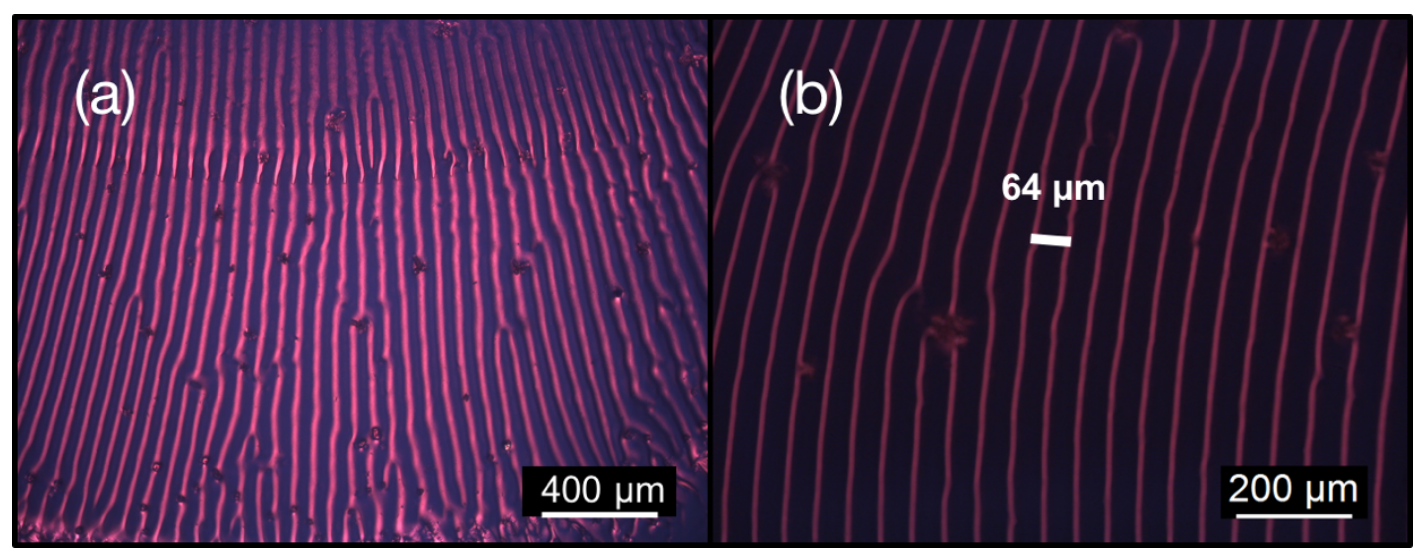

Figure 44. Optical images of periodic stripe textures of P3C5T in DMSO at (a) 5x and (b) 10x magnification showing the periodicity of P3C5T banded textures. 
A sufficiently swollen and oriented polythiophene gel can provide a worthwhile preliminary material for doping of electrically conductive films ${ }^{62}$. Moreover, the presence of the LC phase implies that enhanced alignment of the P3C5T gel network may be possible, based off previous observations from system 1. Estimates of the LC phase boundary and minimum gelation concentration shows both of these values to be of similar order ( 21 wt\%). As a result, any P3C5T solution of high enough concentration to form a nematic LC phase will simultaneously form a gel. If the time required to form the gel state is kinetically favored over the time required for the polymer chains to align, then the final state of the P3C5T system will be isotropic. Therefore, alignment of P3C5T chains should be induced just prior to overall gelation of the system. Figure 44 displays the alignment of P3C5T thin films in response to an applied mechanical shear force. After heating and subsequent slow cooling of the P3C5T gel, a shear force was applied across the still-wet polymeric samples. At the lowest magnification, POM images of sheared P3C5T thin films illustrate the existence of planar alignment in the LC domains. When the shear direction is in line with the crossed polarizers, minimal transmission intensity is detected through the POM (Figure 45a). After rotation of the polarizer by $45^{\circ}$, transmission intensity of the sample is augmented (Figure 45b). This provides significant evidence that the LC director's azimuthal angle is not degenerate, but rather well defined over thousands of micrometers within the substrate plane. 

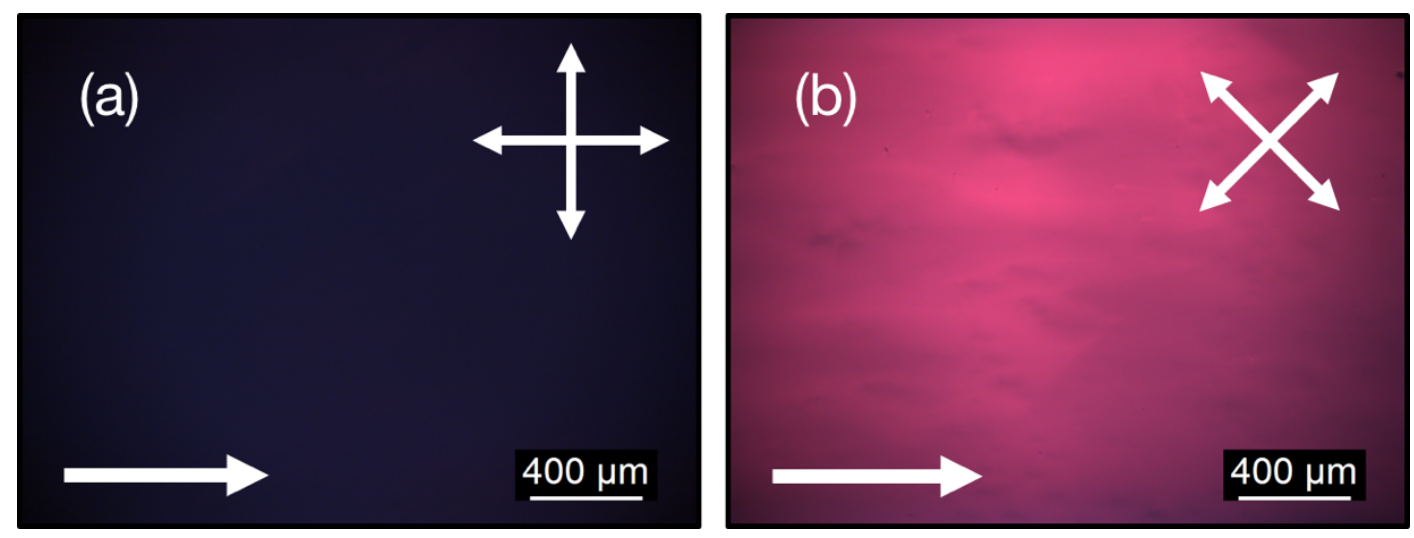

Figure 45. Optical images of sheared films of gelled P3C5T in DMSO under crossed polarizers. (a) $0^{\circ}$ rotation and (b) $45^{\circ}$ rotation of crossed polarizers. The solution concentration is $23 \mathrm{wt} \%$ and the shear direction is parallel to the single arrows.

\subsubsection{Optoelectronic Anisotropy of P3C5T Thin Films}

The in-plane optical anisotropy of aligned P3C5T LC gels is quantified in Figure 46. The degree of order inherent in the system is determined through the distribution symmetry of transmission intensity plots. Through rotation of the P3C5T films in $15^{\circ}$ increments, gradual alterations of transmission intensity are observed (Figure 46a). As such, the intensity of these images are plotted as a function of rotational degrees in Cartesian coordinates (Figure 46b). The resultant plot supports an overall Gaussian-like symmetry and displays minimum transmission intensity with the P3C5T long axis parallel and perpendicular to the polarizer $\left(0^{\circ}\right.$ or $\left.90^{\circ}\right)$. A maximum intensity is reached at an angle of $45^{\circ}$. These findings are consistent with previous observations made regarding Figure 45. To further demonstrate the optical anisotropy of aligned P3C5T films, transmission 
intensity plots were constructed in polar coordinates over a rotational range of $360^{\circ}$. A characteristic and highly symmetric flower pedal shape is observed, denoting a significant optical anisotropy of the system ${ }^{108}$.

(a)
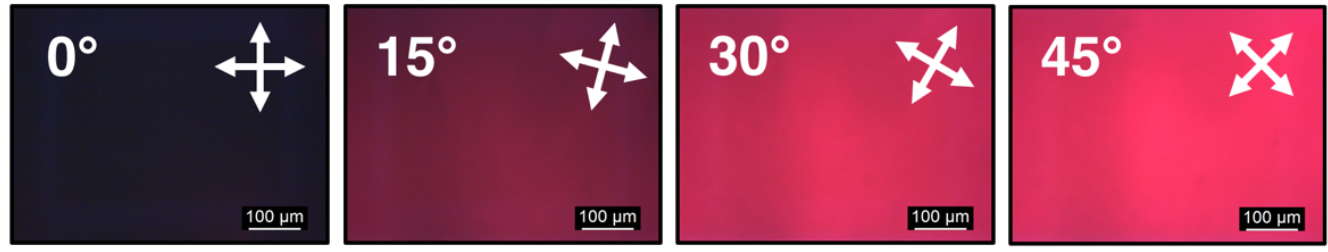

(b)

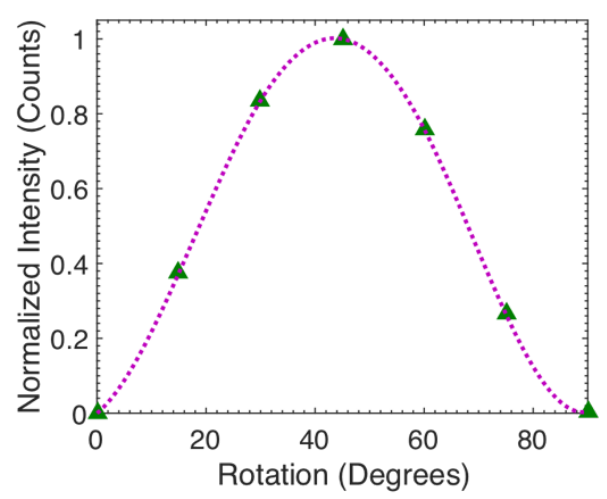

(c)

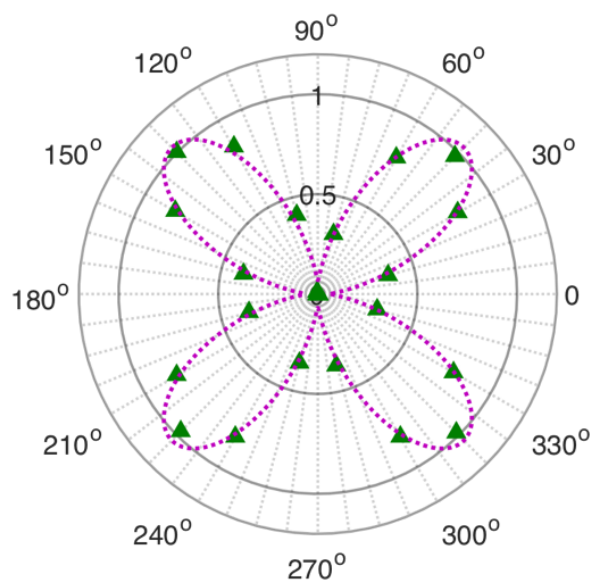

Figure 46. (a) Optical images showing alterations of transmission intensity of sheared P3C5T films as a function of sample rotation. Plots of Transmission intensity as a function of sample rotation in (b) Cartesian and (c) polar coordinates illustrating optical anisotropy of sheared P3C5T LC gel films. For both plots, normalization was done over the entire range of data shown.

Promotion of LC gelation provides a facile way to foster large-area thin film alignment of conjugated polymers with enhanced anisotropic properties for future electronic and energy applications. After air drying of the sheared P3C5T gel films, aligned LC monodomains were maintained. Electrical conductivities of the films 
were measured at room temperature in a four-point probe configuration. Figure 47 presents I-V curves of P3C5T thin films and conductivity values with probes parallel and perpendicular to the shear direction. Directional conductivity measurements of the P3C5T films demonstrate a clear electronic anisotropy. Careful calculations show values of the electrical conductivities, both parallel and perpendicular to the direction of shear, to be of $5.23( \pm 1.75) \times 10^{-3}$ and $2.29( \pm$ $0.76) \times 10^{-3} \mathrm{~S} / \mathrm{m}$, respectively. As the long axis (conjugated backbone) of P3C5T chains are oriented parallel to the shear direction, greater charge transport is observed through intramolecular pathways as opposed to intermolecular hopping through $\pi-\pi$ stacking, similar to system 1 . Error in the conductivity values are associated with instrumental limitations for measuring film thickness. Regardless, the uniformity of relative film thickness between measurements suggests the difference in conductivity between parallel and perpendicular orientations is accurate. Moreover, these results show comparable conductivities to that of nondoped P3HT films at $2.85 \times 10^{-3} \mathrm{~S} / \mathrm{m}^{96}$. 
(a)

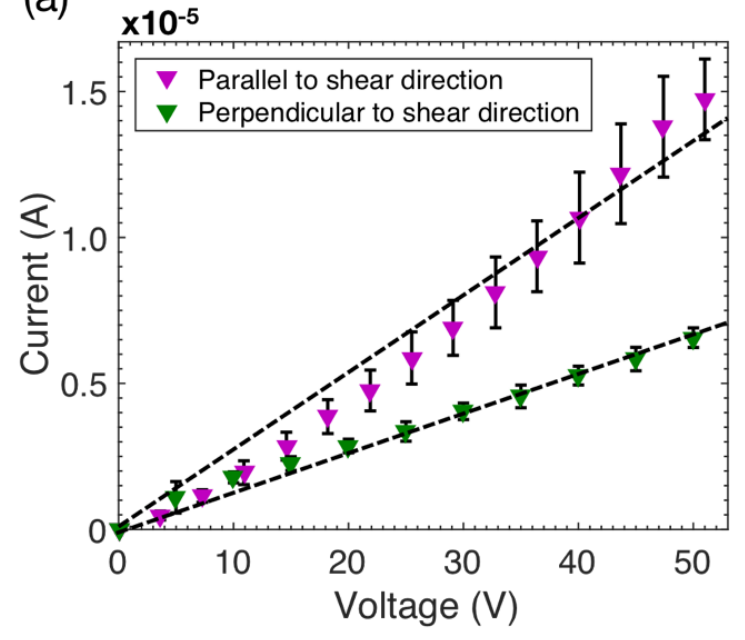

(b)

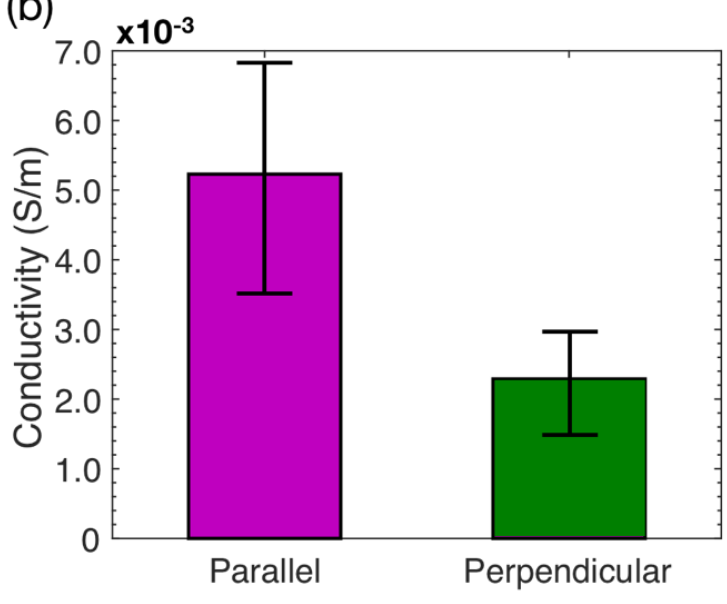

Figure 47. Room-temperature measurement of anisotropic conductivity. (a) Current versus voltage curves of sheared P3C5T LC gel films after complete drying with probes parallel and perpendicular to the shear direction. (b) Bar graph comparing the anisotropic conductivity of P3C5T LC gel films in parallel and perpendicular orientations relative to the direction of shear.

\subsection{Research Outlook and Future Work}

To fully determine whether these methodologies may be generalized to various systems, a compound not known to sustain liquid crystalline characteristics should be investigated. To these ends, P3HT rarely displays liquid crystalline behavior, and the compounds dilute solution assembly is not known to follow inverse first order or second order kinetics. However, the kinetics of $\mathrm{P} 3 \mathrm{HT}$ in the presence of single-walled carbon nanotubes (SWCNT) and a marginal solvent has been investigated previously ${ }^{34}$. The findings demonstrate the rate law of P3HT to increase with the addition of SWCNTs into the system. It is demonstrated that SWCNTs serve as an orientation template and heterogeneous nucleation site for 
$\mathrm{P} 3 \mathrm{HT}$ assembly through $\pi-\pi$ stacking. Therefore, the increased reaction order may be attributed to an expansive proportion of aggregation in the system. As such, the addition of SWCNTs increases the intrinsic weighing factor of aggregation from equation 2. This suggests processing alterations of P3HT systems may be able to influence the assembly kinetics, perhaps leading to LC formation and enhanced morphology of solid state thin films. Modifications such as alkyl-chain length, solvent composition, and changes in concentration should be investigated.

In theory, it may be possible that the intrinsic weighing factors dictating $\mathrm{P} 3 \mathrm{HT}$ assembly are influenced heavily by concentration, and therefore a balance of intermolecular attraction and alkyl-chain steric repulsion. In the case of 1:1 P3K6T-STS ${ }_{16}$, no alterations of intrinsic weighing factors were observed at higher concentrations. However, the experimental concentration range was limited due to poor signal-to-noise ratios above $0.60 \mathrm{mM}$. In the case of system 1 , noise was observed frequently during kinetics measurements. This could be a manifestation of long surfactant alkyl-chains, which do not readily absorb light in the visible region of the electromagnetic spectrum. In contrast, this noise was not observed for kinetics measurements of system 2, which did not contain surfactant molecules. This implies little noise would be present for higher concentrations of system 2 , and therefore $\mathrm{P} 3 \mathrm{HT}$. As such, kinetics measurements of $\mathrm{P} 3 \mathrm{HT}$ should be investigated at concentrations near the instrumental limits of detection, allowing one to determine if any variation in the transition exponent is evident. 


\section{Conclusions}

In this work, multi-scale assembly methodologies of poly(3-hexylthiophene) derivative systems have been investigated. For system 1 , the assembly behavior of P3KnTs complexed with aqueous quaternary ammonium surfactants was investigated at different length scales. In diluted solutions, the supramolecular complexes undergo coil-to-rod transitions of an intramolecular mechanism. Spectroscopic examination of the dynamic process reveals a universal inverse first-order rate law of the transition. The surfactant length and architecture effectively tune the persistence length and the effective conjugation length of the supramolecular complexes. As such, the intrinsic rigidity of conjugated polymers is determinative to accelerate rod-like structures. In concentrated solutions, the supramolecular complexes exhibit ordered liquid crystalline phases above a critical concentration. SAXS measurements are suggestive of hexagonally packed rodlike polymer chains. The combination of mechanical shearing with liquid crystallinity results in aligned thin films at macroscopic scales, which shows approximately four times faster charge transport along the backbone alignment direction than the perpendicular direction.

For system 2, the assembly behavior of a carboxylic acid functionalized poly(3-alkythiophene) derivative (P3C5T) was investigated in diluted and concentrated solutions. In dilute solutions, a combination of good and poor solvents is found to promote P3C5T assembly. With increasing temperature, such self-organizations are thermodynamically hindered, indicating the solution assembly process is thermally reversible. Thermally-induced spectroscopic 
transitions display second order growth kinetics, signifying the solution assembly is of an intermolecular mechanism promoted through the presence of hydrogen bonded carboxylic acid side-chains. In concentrated solutions, hydrogen bonding promotes thermoreversible gelation above a critical concentration. Under an appropriate balance of mechanical and thermal stresses, nematic LC phases displaying Schlerien textures are observed. By thermally weakening the gel networks, mechanical shearing of still-wet P3C5T films induces alignment of the LC phase at macroscopic scales. The aligned films of P3C5T LC gels display a significant anisotropy of optical and electrical properties, with more than two times faster charge transport along the backbone alignment direction compared to the perpendicular direction.

For system 1, surfactant complexation seeks to combine the alignment capacity of LCs with the environmentally friendly benefits of aqueous processing. For system 2, the use of short-range intermolecular interactions yields a simplistic route to merging the processing potential of conjugated polymer gels with the alignment capabilities of LCs in a high dielectric medium. Overall, these methodologies may provide a facile pathway to the production of environmentally friendly high performance optoelectronic devices. 
Bibliography

(1) Fredga, A. Award Ceremony Speech

http://www.nobelprize.org/nobel_prizes/chemistry/laureates/1953/press.ht $\mathrm{ml}$ (accessed Jun 28, 2017).

(2) American Chemical Society National Historic Chemical Landmarks http://www.acs.org/content/acs/en/education/whatischemistry/landmarks/ca rotherspolymers.html (accessed Jun 28, 2017).

(3) Ziegler, K. Consequences and development of an invention* http://www.nobelprize.org/nobel_prizes/chemistry/laureates/1963/zieglerlecture.html (accessed Jun 28, 2017).

(4) Flory, P. J. Nobel Lecture: Spatial Configuration of Macromolecular Chains http://www.nobelprize.org/nobel_prizes/chemistry/laureates/1974/florylecture.html (accessed Jun 28, 2017).

(5) Shirakawa, H.; Louis, E. J.; MacDiarmid, A. G.; Chiang, C. K.; Heeger, A. J. Synthesis of Electrically Conducting Organic Polymers: Derivatives of Polyacetylene. 1977, No. 578, 578-580.

(6) Heeger, A. J. Nobel Lecture: Semiconducting and Metallic Polymers: The Fourth Generation of Polymeric Materials. http://www.nobelprize.org/nobel_prizes/chemistry/laureates/2000/heegerlecture.html (accessed Jun 28, 2017).

(7) Woo, Y.; Heeger, A. J. Conducting Polymers for Carbon Electronics Themed Issue Semiconducting Polymers: The Third Generation W. Chem. Soc. Rev. 2010, 39 (7), 2354-2371.

(8) Dou, L.; You, J.; Hong, Z.; Xu, Z.; Li, G.; Street, R. A.; Yang, Y. 25th Anniversary Article: A Decade of Organic/polymeric Photovoltaic Research. Adv. Mater. 2013, 25 (46), 6642-6671.

(9) Vezie, M. S.; Few, S.; Meager, I.; Pieridou, G.; Dörling, B.; Ashraf, R. S.; Goñi, A. R.; Bronstein, H.; Mcculloch, I.; Hayes, S. C.; Campoy-quiles, M.; Nelson, J. Exploring the Origin of High Optical Abosrption in Conjugated Polymers. Nat. Mater. 2016, No. May, 1-9.

(10) Scholes, G. D.; Rumbles, G. Excitons in Nanoscale Systems. Nat. Mater. 2006, 5, 1-7.

(11) Krebs, F. C.; Søndergaard, R. R.; Markus, H. Roll-to-Roll Fabrication of Large Area Functional Organic Materials. J. Polym. Sci. Part B Polym. Phys. 2013, 51, 16-34. 
(12) Deibel, C.; Dyakonov, V. Polymer - Fullerene Bulk Heterojunction Solar Cells. Rep. Prog. Phys. 2010, No. 73, 1-39.

(13) Dam, H. F.; Larsen-Olsen, T. T. The Layer Stack http://www.plasticphotovoltaics.org/lc/lc-polymersolarcells/lc-layer.html (accessed Jul 6, 2017).

(14) Pröller, S.; Liu, F.; Zhu, C.; Wang, C.; Russell, T. P.; Hexemer, A.; MüllerBuschbaum, P.; Herzig, E. M. Following the Morphology Formation In Situ in Printed Active Layers for Organic Solar Cells. Adv. Energy Mater. 2016, $6(1), 1-9$.

(15) Green, M. A.; Emery, K.; Hishikawa, Y.; Warta, W.; Dunlop, E. D. Solar Cell Efficiency Tables (Version 39). Prog. Photovolt. Res. Appl. 2012, 20 , 12-20.

(16) Chung, K.; Yu, Y.; Kwon, M. S.; Swets, J.; Kim, J.; Youk, J. H. Assembly and Alignment of Conjugated Polymers: Materials Design, Processing, and Applications. MRS Commun. 2015, 5 (02), 169-189.

(17) Kim, J. S.; Lee, J. H.; Park, J. H.; Shim, C.; Sim, M.; Cho, K. HighEfficiency Organic Solar Cells Based on Preformed poly(3Hexylthiophene) Nanowires. Adv. Funct. Mater. 2011, 21 (3), 480-486.

(18) Scharber, M. C.; Sariciftci, N. S. Efficiency of Bulk-Heterojunction Organic Solar Cells. Prog. Polym. Sci. 2013, 38 (12), 1-13.

(19) Le, T. H.; Kim, Y.; Yoon, H. Electrical and Electrochemical Properties of Conducting Polymers. Polymers (Basel). 2017, 9 (150), 1-32.

(20) Mccullough, R. D.; Tristram-nagle, S.; Williams, S. P.; Lowevt, R. D. SelfOrienting Head-to-Tail Poly( 3-Alkylthiophenes): New Insights on StructureProperty Relationships in Conducting Polymers. J. Am. Chem. Soc. 1993, No. $115,4910-4911$.

(21) Yao, Y.; Dong, H.; Liu, F.; Russell, T. P.; Hu, W. Approaching Intra- and Interchain Charge Transport of Conjugated Polymers Facilely by Topochemical Polymerized Single Crystals. Adv. Mater. 2017, 1701251, $1-$ 6.

(22) Roux, C.; Leclerc, M. Rod-to-Coil Transition in Alkoxy-Substituted Polythiophenes. Macromolecules 1992, 25, 2141-2144.

(23) Huang, Y.; Kramer, E. J.; Heeger, A. J.; Bazan, G. C. Bulk Heterojunction Solar Cells : Morphology and Performance Relationships. Chem. Rev. 2014, No. 114, 7006-7043. 
(24) Photovoltaics for the Next Generation: Organic-Based Solar Cells Part 1 http://what-when-how.com/nanoscience-andnanotechnology/photovoltaics-for-the-next-generation-organic-based-solarcells-part-1-nanotechnology/ (accessed Jun 28, 2017).

(25) Jeffries-El, M.; Sauvé, G.; McCullough, R. D. Facile Synthesis of EndFunctionalized Regioregular poly(3-Alkylthiophene)s via Modified Grignard Metathesis Reaction. Macromolecules 2005, 38 (25), 10346-10352.

(26) Osaka, I.; Mccullough, R. D. Advances in Molecular Design and Synthesis of Regioregular Polythiophenes. Acc. Chem. Res. 2008, 41 (9), 12021214.

(27) Binh, N. T.; Gaflberger, M.; Bfissler, H. Photoconduction in Poly ( 3Alkylthiophene ) I . Charge Carrier Generation. Synth. Met. 1992, 47, 7786.

(28) Roehling, J. D.; Arslan, I.; Moulé, A. J. Controlling Microstructure in poly(3Hexylthiophene) Nanofibers. J. Mater. Chem. 2012, 22 (6), 2498-2506.

(29) Sun, S.; Salim, T.; Wong, L. H.; Foo, Y. L.; Boey, F.; Lam, Y. M. A New Insight into Controlling poly(3-Hexylthiophene) Nanofiber Growth through a Mixed-Solvent Approach for Organic Photovoltaics Applications. J. Mater. Chem. 2011, 21 (2), 377-386.

(30) Xu, W.; Li, L.; Tang, H.; Li, H.; Zhao, X.; Yang, X. Solvent-Induced Crystallization of Poly ( 3 -Dodecylthiophene ): Morphology and Kinetics. J. Phys. Chem. B 2011, No. 115, 6412-6420.

(31) Luo, Y.; Santos, F. A.; Wagner, T. W.; Tsoi, E.; Zhang, S. Dynamic Interactions between poly(3-Hexylthiophene) and Single-Walled Carbon Nanotubes in Marginal Solvent. J. Phys. Chem. B 2014, 118 (22), 60386046 .

(32) Chu, B.; Xu, R. Chromatic Transition of Polydiacetylene in Solution. Acc. Chem. Res. 1991, 24 (12), 384-389.

(33) Rughooputh, S.; Hotta, S. Chromism of Soluble Polythienylenes. J. Polym. ... 1987, 25, 1071-1078.

(34) Luo, Y.; Santos, F. A.; Wagner, T. W.; Tsoi, E.; Zhang, S. Dynamic Interactions between Poly(3-Hexylthiophene) and Single- Walled Carbon Nanotubes in Marginal Solvent. J. Phys. Chem. B 2014, No. 118, 60386046. 
(35) Wagner, T. W.; Luo, Y.; Redeker, N. D.; Immoos, C. E.; Zhang, S. Effect of Surface-Modified Zinc Oxide Nanowires on Solution Crystallization Kinetics of poly(3-Hexylthiophene). Polymer (Guildf). 2014, 55 (8), 2008-2013.

(36) Lagerwall, J. P. F.; Scalia, G.; Scalia, G. A New Era for Liquid Crystal Research: Applications of Liquid Crystals in Soft Matter Nano- , Bio- and Microtechnology. Curr. Appl. Phys. 2012, 12 (6), 1387-1412.

(37) What is a Liquid Crystal? https://www.quora.com/What-is-a-liquid-crystal (accessed Jul 6, 2017).

(38) Lyotropic Liquid Crystals https://en.wikipedia.org/wiki/Lyotropic_liquid_crystal (accessed Jul 6, 2017).

(39) Yao, J.; Bastiaansen, C. W. M.; Peijs, T. High Strength and High Modulus Electrospun Nanofibers. Fibers 2014, No. 2, 158-187.

(40) Kim, B.-G.; Jeong, E. J.; Chung, J. W.; Seo, S.; Koo, B.; Kim, J. A Molecular Design Principle of Lyotropic Liquid-Crystalline Conjugated Polymers with Directed Alignment Capability for Plastic Electronics. Nat Mater 2013, 12 (7), 659-664.

(41) Kumar, M.; Kumar, S. Liquid Crystals in Photovoltaics: A New Generation of Organic Photovoltaics. Polym. J. 2017, 49 (1), 85-111.

(42) O'Neill, M.; Kelly, S. M. Ordered Materials for Organic Electronics and Photonics. Adv. Mater. 2011, 23 (5), 566-584.

(43) Liu, C.; Tan, Y.; Li, C.; Wu, F.; Chen, L.; Chen, Y. Enhanced PowerConversion Efficiency in Inverted Bulk Heterojunction Solar Cells Using Liquid-Crystal-Conjugated Polyelectrolyte Interlayer. ACS Appl. Mater. Interfaces 2015, 7 (34), 19024-19033.

(44) Frenkel, D. Perspective on "The Effect of Shape on the Interaction of Colloidal Particles" - Onsager L (1949) Ann NY Acad Sci 51 : 627. Theor. Chem. Acc. 2000, 103, 212-213.

(45) Zhu, Z.; Swager, T. M. Conjugated Polymer Liquid Crystal Solutions : Control of Conformation and Alignment. J. Am. Chem. Soc. 2002, No. 124, 9670-9671.

(46) Liquid Crystal https://en.wikipedia.org/wiki/Liquid_crystal (accessed Jul 6, 2017). 
(47) Newbloom, G. M.; Weigandt, K. M.; Pozzo, D. C. Electrical, Mechanical, and Structural Characterization of Self-Assembly in poly(3-Hexylthiophene) Organogel Networks. Macromolecules 2012, 45 (8), 3452-3462.

(48) Kim, B.; Jeong, E. J.; Park, H. J.; Bilby, D.; Guo, L. J.; Kim, J. Effect of Polymer Aggregation on the Open Circuit Voltage in Organic Photovoltaic Cells : Aggregation-Induced Conjugated Polymer Gel and Its Application for Preventing Open Circuit Voltage Drop. Appl. Mater. Interfaces 2011, No. 3, 674-680.

(49) Ajayaghosh, A.; Babu, S. S.; Prasanthkumar, S.; Ajayaghosh, A. SelfAssembled Gelators for Organic Electronics. Angew. Chemie - Int. Ed. 2012, No. 51, 1766-1776.

(50) Koppe, M.; Brabec, C. J.; Duffy, W.; Heeney, M.; Mcculloch, I. Influence of Molecular Weight Distribution on the Gelation of P3HT and Its Impact on the Photovoltaic Performance. Macromolecules 2009, No. 42, 4661-4666.

(51) Malik, S.; Nandi, A. K. Influence of Alkyl Chain Length on the Gelation Mechanism of Thermoreversible Gels of Regioregular Poly ( 3-Alkyl Thiophenes ) in Xylene. J. Appl. Polym. Sci. 2006, 2528-2537.

(52) Newbloom, G. M.; de la Iglesia, P.; Pozzo, L. D. Controlled Gelation of poly(3-Alkylthiophene)s in Bulk and in Thin-Films Using Low Volatility Solvent/poor-Solvent Mixtures. Soft Matter 2014, 10 (44), 8945-8954.

(53) Oh, J. H.; Kim, B. J.; Woo, H. Y. Ethanol-Processable, Highly Crystalline Conjugated Polymers for Eco-Friendly Fabrication of Organic Transistors and Solar Cells. Macromolecules 2017, No. 50, 4415-4424.

(54) Chen, X.; Liu, X.; Burgers, M. A.; Huang, Y.; Bazan, G. C. Green-SolventProcessed Molecular Solar Cells ${ }^{* *}$. Angew. Chemie - Int. Ed. 2014, No. 53, 14378-14381.

(55) Chueh, C.; Yao, K.; Yip, H.; Chang, C.; Xu, Y.; Chen, K.; Li, C.; Liu, P.; Huang, F.; Chen, Y.; Chen, W.; Jen, A. Non-Halogenated Solvents for the Environmental Friendly Processing of High-Performance BulkHeterojunction Polymer Solar Cells. Energy Environ. Sci. 2013, 1-11.

(56) Søndergaard, R.; Helgesen, M.; Jørgensen, M.; Krebs, F. C. Fabrication of Polymer Solar Cells Using Aqueous Processing for All Layers Including the Metal Back Electrode. Adv. Energy Mater. 2011, No. 1, 68-71.

(57) Duarte, A.; Pu, K. Y.; Liu, B.; Bazan, G. C. Recent Advances in Conjugated Polyelectrolytes for Emerging Optoelectronic Applications. Chem. Mater. 2011, 23 (3), 501-515. 
(58) Laurenti, M.; Rubio-retama, J.; Garcia-blanco, F.; Lo, E. Influence of the Surfactant Chain Length on the Fluorescence Properties of a WaterSoluble Conjugated Polymer Influence of the Surfactant Chain Length on the Fluorescence Properties of a Water-Soluble Conjugated Polymer. Society 2008, No. 16, 13321-13327.

(59) Zhang, S.; Pfefferle, L. D.; Osuji, C. O. Lyotropic Hexagonal Ordering in Aqueous Media by Conjugated Hairy-Rod Supramolecules. Macromolecules 2010, 43 (18), 7549-7555.

(60) Worfolk, B. J.; Rider, D. A.; Elias, A. L.; Thomas, M.; Harris, K. D.; Buriak, J. M. Bulk Heterojunction Organic Photovoltaics Based on Carboxylated Polythiophenes and PCBM on Glass and Plastic Substrates. Adv. Funct. Mater. 2011, 21 (10), 1816-1826.

(61) Li, W.; Worfolk, B. J.; Li, P.; Hauger, T. C.; Harris, K. D.; Buriak, J. M. SelfAssembly of Carboxylated Polythiophene Nanowires for Improved Bulk Heterojunction Morphology in Polymer Solar Cells. J. Mater. Chem. 2012, 22 (July 2016), 11354.

(62) Alcazar, D.; Wang, F.; Swager, T. M.; Thomas, E. L. Gel Processing for Highly Oriented Conjugated Polymer Films. Macromolecules 2008, 41 (24), 9863-9868.

(63) Determination of Caffeine and Benzoic Acid in Soft Drinks by Multicomponent UV Analysis http://sites.cord.edu/chem-330-labmanual/experiments/uv-vis (accessed Jul 6, 2017).

(64) Molecular Spectroscopy http://chemistry.tutorvista.com/analyticalchemistry/molecular-spectroscopy.html (accessed Jul 6, 2017).

(65) Hotta, S.; Ito, K. Handbook of Oligo- and Polythiophenes; Fichou, D., Ed.; Wiley-VCH Verlag GmbH: Weinheim, Germany, 1998.

(66) Robinson, P.; Davidson, M. Polarized Light Microscopy https://www.microscopyu.com/techniques/polarized-light/polarized-lightmicroscopy (accessed Jul 6, 2017).

(67) Kumar, J. Resistivity of a Semi-Conductor by the Four-Probe Method. Lab Exp. 2011, 1 (1), 1-14.

(68) Geometric Factors in Four Point Resistivity Measurement http://four-pointprobes.com/haldor-topsoe-geometric-factors-in-four-point-resistivitymeasurement/ (accessed Jul 6, 2017). 
(69) Smits, B. F. M. Measurement of Sheet Resistivities with the Four-Point Probe. Bell Syst. Tech. J. 1958, 711-718.

(70) Four Point Probe http://Inf-wiki.eecs.umich.edu/wiki/Four_point_probe (accessed Jul 6, 2017).

(71) Danesh, C. D.; Starkweather, N. S.; Zhang, S. In Situ Study of Dynamic Conformational Transitions of a Water-Soluble poly(3-Hexylthiophene) Derivative by Surfactant Complexation. J. Phys. Chem. B 2012, 116 (42), 12887-12894.

(72) Lavigne, J. J.; Broughton, D. L.; Wilson, J. N.; Erdogan, B.; Bunz, U. H. F. Communications to the Editor: "Surfactochromic" Conjugated Polymers: Surfactant Effects on Sugar-Substituted PPEs. Macromolecules 2003, 36 (20), 9-12.

(73) Knaapila, M.; Evans, R. C.; Garamus, V. M.; Almásy, L.; Székely, N. K.; Gutacker, A.; Scherf, U.; Burrows, H. D. Structure and "Surfactochromic" Properties of Conjugated Polyelectrolyte (CPE): Surfactant Complexes between a Cationic Polythiophene and SDS in Water. Langmuir 2010, 26 (19), 15634-15643.

(74) Franco, I. E.; Lorchat, P.; Lamps, J. P.; Schmutz, M.; Schr??der, A.; Catala, J. M.; Combet, J.; Schosseler, F. From Chain Collapse to New Structures: Spectroscopic Properties of poly(3-Thiophene Acetic Acid) upon Binding by Alkyl Trimethylammonium Bromide Surfactants. Langmuir 2012, 28 (10), 4815-4828.

(75) Gettinger, C. L.; Heeger, A. J.; Drake, J. M.; Pine, D. J. A Photoluminescence Study of Poly(phenylene Vinylene) Derivatives: The Effect of Intrinsic Persistence Length. J. Chem. Phys. 1994, 101 (2), 1673.

(76) Benincori, T.; Consonni, V.; Gramatica, P.; Pilati, T.; Rizzo, S.; Sannicolo, F.; Todeschini, R.; Zotti, G. Steric Control of Conductivity in Highly Conjugated Polythiophenes. Chem. Mater. 2001, 13 (5), 1665-1673.

(77) Meng, K.; Ding, Q.; Wang, S.; He, Y.; Li, Y.; Gong, Q. Spatial Conformation and Charge Recombination Properties of Polythiophene Deriatives With Thienylene-Vinylene Side Chains Investigated by Static and Femtosecond Spectroscopy. J. Phys. Chem. B 2010, 114 (8), 2602-2606.

(78) Geochemistry, P. IR Spectroscopy of Organic Acids [ölihttp://geoweb.princeton.edu/research/geochemistry/research/organicscarboxyl.html (accessed Jul 6, 2017). 
(79) Garcia, A.; Nguyen, T. Effect of Aggregation on the Optical and Charge Transport Properties of an Anionic Conjugated Polyelectrolyte. J. Phys. Chem. C 2008, 112, 7054-7061.

(80) Evans, R. C.; Knaapila, M.; Willis-Fox, N.; Kraft, M.; Terry, A.; Burrows, H. D.; Scherf, U. Cationic polythiop1. Evans, R. C. et Al. Cationic Polythiophene-Surfactant Self-Assembly Complexes: Phase Transitions, Optical Response, and Sensing. Langmuir 28, 12348-56 (2012).heneSurfactant Self-Assembly Complexes: Phase Transitions, Optical Response. Langmuir 2012, 28 (33), 12348-12356.

(81) Rous, C.; Leclerc, M. Rod-to-Coil Transition in Alkoxy-Substituted Polythiophenes. Macromolecules 1992, 25 (8), 2141-2144.

(82) Sundberg, M.; Inganas, O.; Stafstrom, S.; Gustafsson, G.; Sjogren, B. Optical Absorption of poly(3-Alkylthiophenes) at Low Temperatures. Solid State Commun. 2016, 71 (6), 435-439.

(83) Hall, K. J.; Quickenden, T. I.; Watts, D. W. Rate Constants from Initial Concentration Data. J. Chem. Educ. 1976, 53 (8), 493.

(84) Lecommandoux, S.; Chécot, F.; Borsali, R.; Schappacher, M.; Deffieux, A.; Brûlet, A.; Cotton, J. P. Effect of Dense Grafting on the Backbone Conformation of Bottlebrush Polymers: Determination of the Persistence Length in Solution. Macromolecules 2002, 35 (23), 8878-8881.

(85) Roe, R.-J. Methods of X-Ray and Neutron Scattering in Polymer Science; 2000.

(86) Choudhury, P. K.; Bagchi, D.; Sangeeth, C. S. S.; Menon, R. Modified Conformation and Physical Properties in Conducting Polymers due to Varying Conjugation and Solvent Interactions. J. Mater. Chem. 2010, 21, 1607-1614.

(87) Li, Y.-C.; Chen, K.-B.; Chen, H.-L.; Hsu, C.-S.; Tsao, C.-S.; Chen, J.-H.; Chen, S.-A. Fractal Aggregates of Conjugated Polymer in Solution State. Langmuir 2006, 22 (26), 11009-11015.

(88) Rodd, C. M.; Agarwal, R. Enha(1) Rodd, C. M.; Agarwal, R. Nano Lett. 2011, 11 (8), 3460-3467.ncement of Interfacial Polymer Crystallinity Using Chromism in Single Inorganic Nanowire-Polymer Nanohybrids for Photovoltaic Applications. Nano Lett. 2011, 11 (8), 3460-3467.

(89) Khokhlov, A. R.; Semenov, A. N. Liquid-Crystalline Ordering in the Solution of Long Persistent Chains. Phys. A Stat. Mech. its Appl. 1981, 108 (2-3), 546-556. 
(90) Zhang, S.; Fu, L.; Zhang, J.; Liu, J.; Yang, D.; Ge, J. J.; Li, C. Y.; Cheng, S. $Z$. D. Ordering-Induced Micro-Bands in Thin Films of a Main-Chain Liquid Crystalline Chloro-Poly(aryl Ether Ketone). Polymer (Guildf). 2004, 45 (11), 3967-3972.

(91) Klaiber, A.; Lanz, C.; Landsmann, S.; Gehring, J.; Drechsler, M.; Polarz, S. Maximizing Headgroup Repulsion: Hybrid Surfactants with Ultrahighly Charged Inorganic Heads and Their Unusual Self- Assembly. Langmuir 2016, 32, 10920-10927.

(92) Choo, Y.; Mahajan, L. H.; Gopinadhan, M.; Ndaya, D.; Deshmukh, P.; Kasi, R. M.; Osuji, C. O. Phase Behavior of Polylactide-Based Liquid Crystalline Brushlike Block Copolymers. Macromolecules 2015, 48 (22), 8315-8322.

(93) Yang, S.; Hsu, C. Liquid Crystalline Conjugated Polymers and Their. J. Polym. Sci. Part A Polym. Chem. 2009, 47, 2713-2733.

(94) Bates, M. A.; Frenkel, D. Influence of Polydispersity on the Phase Behavior of Colloidal Liquid Crystals : A Monte Carlo Simulation Study. J. Chem. Phys. 1998, 109 (14), 6193-6199.

(95) Selinger, Jonathan V. and Bruinsma, R. F. Hexagonal and Nematic Phases of Chains. II. Phase Transitions. Phys. Rev. A 1991, 43 (6), 2922-2931.

(96) Kuila, B. K.; Malik, S.; Batabyal, S. K.; Nandi, A. K. In-Situ Synthesis of Soluble poly(3-Hexylthiophene)/multiwalled Carbon Nanotube Composite: Morphology, Structure, and Conductivity. Macromolecules 2007, 40 (2), 278-287.

(97) Eslamibidgoli, M. J.; Lagowski, J. B. The Effect of Side-Chain Length on the Solid-State Structure and Optoelectronic Properties of Fluorene- Alt Benzothiadiazole Based Conjugated Polymers-A DFT Study. J. Phys. Chem. A 2012, 116 (43), 10597-10606.

(98) Cox, R.; Olson, G. T.; Pfau, M.; Eshaghi, N.; Barcus, K.; Ramirez, D.; Fernando, R.; Zhang, S. Solution-Based Large-Area Assembly of Coaxial Inorganic-Organic Hybrid Nanowires for Fast Ambipolar Charge Transport. 2017, 3-9.

(99) Xie, H.; O'Dwyer, S.; Corish, J.; Morton-Blake, D. A. The Thermochromism of poly(3-Alkylthiophene)s: The Role of the Side Chains. Synth. Met. 2001, 122 (2), 287-296.

(100) Vinokurov, I. a; Kankare, J. Beer's Law and the Isosbestic Points in the Absorption Spectra of Conductive Polymers. J. Phys. Chem. B 1998, 102 (97), 1136-1140. 
(101) Dierckx, W.; Oosterbaan, W. D.; Bolsée, J.-C.; Maes, W.; Vanderzande, D.; Manca, J. Poly(3-Alkylthiophene) Nanofibers for Optoelectronic Devices. J. Mater. Chem. C 2014, 2 (29), 5730.

(102) Philipse, A. P.; Wierenga, A. M. On the Density and Structure Formation in Gels and Clusters of Colloidal Rods and Fibers. Langmuir 1998, 14 (1), 49-54.

(103) Bertinelli, F.; DellaCasa, C. Solvatochromism in Poly 3-(10-Hydroxydecyl) Thiophene: A Spectrokinetic Study of the Conformational Transition. Polymer (Guildf). 1996, 37 (24), 5469-5475.

(104) Jones, M. M.; Gilkerson, W. R.; Gallup, G. A. Hydrogen Bond Energies in Carboxylic Acids. J. Chem. Phys. 1952, 20 (6), 1048.

(105) Sun, K.; Xiao, Z.; Lu, S.; Zajaczkowski, W.; Pisula, W.; Hanssen, E.; White, J. M.; Williamson, R. M.; Subbiah, J.; Ouyang, J.; Holmes, A. B.; Wong, W. W. H.; Jones, D. J. A Molecular Nematic Liquid Crystalline Material for High-Performance Organic Photovoltaics. Nat. Commun. 2015, 6, 6013.

(106) Casalnuovo, S. A.; Heeger, A. J. Spontaneous Nematic Alignment in Conjugated-Polymer Gels. Phys. Rev. Lett. 1984, 53 (23).

(107) Kleinhenz, N.; Rosu, C.; Chatterjee, S.; Chang, M.; Nayani, K.; Xue, Z.; Kim, E.; Middlebrooks, J.; Russo, P. S.; Park, J. O.; Srinivasarao, M.; Reichmanis, E. Liquid Crystalline poly(3-Hexylthiophene) Solutions Revisited: Role of Time-Dependent Self-Assembly. Chem. Mater. 2015, 27 (7), 2687-2694.

(108) Lu, G.; Li, G.; Li, S.; Qu, Y.; Tang, H.; Yang, X. Constructing Thin Polythiophene Film Composed of Aligned Lamellae via Controlled Solvent Vapor Treatment. Langmuir 2009, 25 (6), 3763-3768. 